\title{
Similarity Relations of Wind Waves in Finite Depth
}

by

\section{Padmaraj Vengayil}

B.Tech., Indian Institute of Technology, Madras, India (1984)

M.S., University of Florida (1986)

Submitted in partial fulfillment of the

requirements for the degree of

MASTER OF SCIENCE

at the

MASSACHUSETTS INSTITUTE OF TECHNOLOGY

and the

WOODS HOLE OCEANOGRAPHIC INSTITUTION

August 1988

(C) Padmaraj Vengayil, 1988

The author hereby grants to MIT and WHOI permission to reproduce anc to distribute copies of this thesis document in whole or in part.

Signature of Author

Joint Program in Oceanographic Engineering Massachusetts Institute of Technology

Woods Hole Oceanographic Institution August 31, 1988

Certified by

Dr. Hans C. Graber

Woods Hole Oceanographic Institution Thesis Supervisor

Certified by

Prof. Ole S. Madsen Massachusetts Institute of Technology

Accepted by

Prof. W. Kendall Melville

Chairman, Joint Committee for Oceanographic Engineering Massachusetts Institute of Technology/Woods Hole Oceanographic Institution 


\title{
Similarity Relations of Wind Waves in Finite Depth
}

by

\author{
Padmaraj Vengayil
}
Submitted to the Massachusetts Institute of Technology/
Woods Hole Oceanographic Institution
Joint Program in Oceanographic Engineering
on August 5, 1988, in partial fulfillment of the
requirements for the degree of
Master of Science

\begin{abstract}
Three formulations for the rear face of a growing wind-sea spectrum in finite depth based on Phillips (1958), Toba (1972) and Donelan et al. (1985) are tested using spectral measurements from Lake St. Clair. Assuming spectral similarity in wave number space relations between the energy, equilibrium parameters and peak wave number are derived. Using a regression analysis, relations are obtained from the data and compared to the theoretical relations. Results indicate that the formulation based on a high-frequency $f^{-4}$ tail (Toba, 1972 and Donelan et al., 1985) is better than the Phillips $f^{-5}$ high-frequency tail. Based on the effective fetch formulation, wave propagation directions are calculated. Relations between the spectral parameters, growth-stage variables and fetch are also determined from the data. The relations indicate a weak dependence of the spectral parameters on depth. Various source terms in the energy balance equation for wave growth in finite depth water are estimated for two cases of wave evolution. The relative importance of wind input, bottom dissipation, white-capping dissipation and nonlinear transfer in the evolution of the spectra is analyzed.
\end{abstract}

Thesis Supervisor: Dr. Hans C. Graber

Woods Hole Oceanographic Institution 


.




\section{Acknowledgements}

I would like to thank my thesis advisor Dr. Hans Graber for his continued support and guidance during the course of this study. His family's friendship is greatly appreciated. I appreciate the support and encouragement of my academic advisor Prof. Ole Madsen. Special thanks to Maxine Jones and Arthur Newhall for help with the computers. Many thanks go to Pat Dixon, Liz Rowe, Wendy Lawrence, Prasanna for their friendship. To

everyone else in Parson's Lab and Joint Program thanks for the interesting time I had in the last two years. And finally I would like to thank my parents for their support.

The work was supported by the WHOI/MIT Education Office and NOAA National Sea Grant College Program Office, Dept. of Commerce under Grant No. NA86-AA-D- SG090, WHOI Sea Grant Project No. R/M -12 . 


\section{Contents}

1 Introduction $\quad \theta$

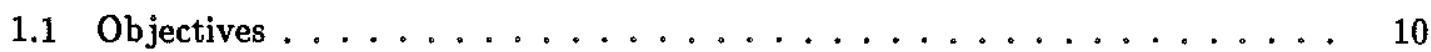

1.2 Equilibrium Range Studies in Deep Water . . . . . . . . . . . 10

1.3 Equilibrium Range Studies in Finite Depth . . . . . . . . . . . 12

1.4 Energy Balance .............................. 12

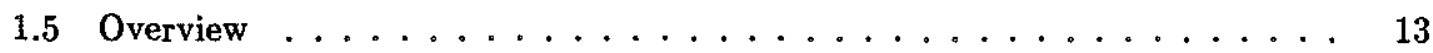

2 Description of the Data Set $\quad 14$

2.1 Meteorological Measurements . . . . . . . . . . . . . . . . . . 14

2.2 Wave Measurements . . . . . . . . . . . . . . . 16

2.3 Summary of Data . . . . . . . . . . . . . . . 16

3 Similarity Relations $\quad 30$

3.1 Peak Wave Direction $\ldots \ldots \ldots \ldots \ldots \ldots \ldots \ldots$

3.2 Equilibrium Range $\ldots \ldots \ldots \ldots \ldots \ldots \ldots \ldots \ldots \ldots$

3.3 Spectral and Growth Stage Parameters . . . . . . . . . . . . . 49

3.4 Fetch Relations . . . . . . . . . . . . . . . . . . . . 54

4 Energy Balance $\quad 68$

4.1 The Energy Transport Equation $\ldots \ldots \ldots \ldots \ldots$ 
4.2 Analysis and Discussion $\ldots \ldots \ldots \ldots \ldots \ldots$

5 Conclusions

6 Referencess 


\section{List of Figures}

2-1 Location of measurement stations in Lake St. Clair . . . . . . . . . . 15

2-2 Time series of measurements at tower $C 1 \ldots \ldots \ldots \ldots \ldots$

$2-3$ Time series of measurements at tower $C 2 \ldots \ldots \ldots \ldots$

$2-4$ Time series of measurements at tower $C 3 \ldots \ldots \ldots \ldots$

$2-5$ Time series of measurements at tower $U 1 \ldots \ldots \ldots \ldots \ldots$

$2-6$ Time series of measurements at tower $U 2 \ldots \ldots \ldots \ldots \ldots \ldots$

$2-7$ Time series of measurements at tower $U 3 \ldots \ldots \ldots \ldots \ldots$

2-8 Time series of spectral parameters at tower $C 1 \ldots \ldots \ldots 24$

$2-9$ Time series of spectral parameters at tower $C 2 \ldots \ldots \ldots$

2-10 Time series of spectral parameters at tower $C 3 \ldots \ldots 26$

2-11 Time series of spectral parameters at tower $U 1 \ldots \ldots \ldots \ldots \ldots$

2-12 Time series of spectral parameters at tower $U 2 \ldots \ldots \ldots \ldots$

2-13 Time series of spectral parameters at tower $U 3 \ldots \ldots \ldots \ldots$

3-1 Observed wind and predicted wave directions at CCIW towers . . . . 32

3-2 Equilibrium parameters as function of frequency $\ldots \ldots \ldots \ldots \ldots$

3-3 Dimensionless energy $\epsilon$ versus $\alpha_{4} \kappa_{m}^{-2}$ for $\omega_{h}<1.0 \ldots \ldots \ldots \ldots$

3-4 Same as Figure 3-3, but $1.0 \leq \omega_{h}<1.3 \ldots \ldots \ldots \ldots$

3-5 Same as Figure $3-3$, but $1.3 \leq \omega_{h}<1.8 \ldots \ldots \ldots \ldots \ldots$ 
3-6 Same as Figure $3-3$, but $\omega_{h} \geq 1.8 \ldots \ldots \ldots \ldots \ldots$

3-7 Dimensionless energy $\epsilon$ versus scaling parameters $\alpha_{4} \kappa_{m}^{-2} \ldots \ldots \ldots . \ldots 42$

3-8 Dimensionless energy $\epsilon$ versus scaling parameters $\frac{u_{*}}{U} \beta \kappa_{m}^{-2} \ldots \ldots \ldots$

3-9 Dimensionless energy $\epsilon$ versus scaling parameters $\alpha_{5} \kappa_{m}^{-2} \ldots \ldots \ldots$

3-10 Dimensionless energy $\epsilon$ versus scaling parameters $\alpha_{4}$ (CCIW towers) . . . 48

3-11 Equilibrium parameter $\alpha_{5}$ versus nondimensional peak frequency $\nu \ldots 53$

3-12 Equilibrium parameter $\beta$ versus nondimensional peak frequency $\nu \ldots \ldots 5$

3-13 Dimensionless energy $\epsilon$ versus relative wind input $U_{e} / c \ldots \ldots \ldots$

3-14 Equilibrium parameter $\alpha_{4}$ versus relative wind input $U_{e} / c \ldots \ldots \ldots$

3-15 Equilibrium parameter $\alpha_{5}$ versus nondimensional wave number $\kappa_{m} \ldots \ldots$

3-16 Dimensionless energy $\epsilon$ versus nondimensional fetch $\xi \ldots \ldots 2$

3-17 Dimensionless peak frequency $\nu$ versus nondimensional fetch $\xi \ldots \ldots 4$

3-18 Equilibrium parameter $\alpha_{5}$ versus nondimensional fetch $\xi \ldots \ldots \ldots$

3-19 Equilibrium parameter $\alpha_{5}$ versus nondimensional fetch $\xi \ldots \ldots 66$

3-20 Equilibrium parameter $\alpha_{5}$ versus nondimensional fetch $\xi \ldots \ldots 67$

4-1 Various source terms contributing to the energy balance for Case $1 \ldots$. . . 77

$4-2$ Same as Figure $4-1$, but for Case $2 \ldots \ldots \ldots \ldots \ldots$

4-3 Various source terms contributing to the energy balance for Case $1 \ldots \ldots 1$

$4-4$ Same as Figure $4-3$, but for Case $2 \ldots \ldots \ldots \ldots$. . . . . . 82 


\section{List of Tables}

3.1 Comparison of regression relations for new data set . . . . . . . . 41

3.2 Regression relations for $\alpha_{5} \ldots \ldots \ldots \ldots \ldots \ldots \ldots \ldots \ldots$

3.3 Regression relations for $\beta \ldots \ldots \ldots \ldots \ldots \ldots \ldots$

3.4 Regression relations for $\alpha_{4} \ldots \ldots \ldots \ldots \ldots \ldots \ldots \ldots$

3.5 Comparison of regression coefficients for the three formulations . . . . . . 49

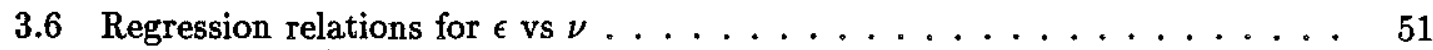

3.7 Regression relations for $\epsilon$ vs $U / c \ldots \ldots \ldots \ldots \ldots \ldots \ldots \ldots \ldots \ldots$

3.8 Regression relations for $\epsilon$ vs $\kappa_{m} \ldots \ldots \ldots \ldots \ldots \ldots$

3.9 Regression relations for $\epsilon$ and $\nu$ versus fetch $\xi \ldots \ldots \ldots$. . . . . . 61

3.10 Regression relations for $\alpha_{4}, \beta$ and $\alpha_{5}$ versus fetch $\xi \ldots \ldots \ldots 1$

4.1 Spectral parameters for Case 1 and Case 2 at the towers . . . . . . 75 


\section{Chapter 1}

\section{Introduction}

Coastal regions are continually altered and modified by nearshore processes. The primary driving force of these processes are surface waves and to understand and model these processes a good knowledge of the evolution of the wind-generated wave field in finite water depth is essential. Observations in deep water have shown that frequency wind-sea spectra are similar in shape and various spectral forms with different shape and scale parameters have been proposed to describe the wind-sea spectrum in deep water. (Phillips, 1958; Pierson and Moskowitz, 1964; Hasselmann et al., 1973; Toba, 1973; Donelan et al., 1985). The various shape and scale parameters have been analyzed in terms of the growth stage parameters and local generating conditions in some of the above studies. Kitaigorodskii et al. (1975) extended the deep water spectral formulation of Phillips (1958) to finite depth and observations by Knowles (1982) and Bouws et al. (1985) have indicated spectral similarity in finite depth. Similarity relations are useful in numerical wave modelling and engineering practice to formulate prediction models that specify the wind-wave field from available measurements of wave and wind conditions. 


\section{$1.1 \quad$ Objectives}

In this study the following issues will be addressed based on wind and wave growth measurements obtained from a field program in Lake St.Clair, a fetch restricted shallow lake on the Canada-US border.

- The differences in the direction of wave propagation and observed wind in fetch limited seas.

- The dependence of the high-frequency portion of the spectrum on the shape parameters of the spectrum.

- Relations between spectral parameters and growth stage parameters.

- Analysis of self-similarity of spectra.

- Spectral growth as a function of fetch.

- The effect of non-concurrence of wind and wave directions on spectral parameters.

- The balance between atmospheric input, nonlinear wave-wave interactions, dissipation by white capping, advection and bottom dissipation in finite depth and the relative importance of these processes in maintaining spectral similarity.

\subsection{Equilibrium Range Studies in Deep Water}

Phillips (1958) argued that above the spectral peak frequency the spectral density is limited by wave breaking due to gravitational instabilities. On dimensional grounds he proposed that for frequencies above the spectral peak the spectral density $E(f)$ follows

$$
E(f)=(2 \pi)^{-4} \alpha_{5} g^{2} f^{-5}
$$


where $g$ is the gravitational acceleration, $f$ is the frequency and $\alpha_{5}$ is a universal constant. This formulation was supported by field measurements of fully grown seas (Pierson and Moskowitz, 1964) and growing seas (Hasselmann et al., 1973, 1976). However, for developing seas it was found that $\alpha_{5}$ varied with growth stage.

Toba (1972) presented a saturation spectral density of the form

$$
E(f)=(2 \pi)^{-3} \beta g u_{*} f^{-4}
$$

where $u_{*}$ is the shear velocity of the wind over the water surface and $\beta$ is a constant. This deviation of the high frequency tail from $f^{-5}$ was observed in laboratory experiments (Toba, 1973) and field measurements (Kawai et al., 1977). Forristal (1981) presented data which supported ( 1.1) for sufficiently high frequencies and (1.2) for lower frequencies of the saturation range. Kahma (1981) also presented support for a formulation similar to (1.2) but with a dependence on wind speed rather than the shear velocity. Donelan et al. (1985) proposed another spectral form similar to (1.2) given by

$$
E(f)=(2 \pi)^{-4} \alpha_{4} g^{2} f_{m}^{-1} f^{-4}
$$

Comparing (1.2) and (1.3) it is seen that

$$
\beta=\left(\frac{2 \pi f_{m} u_{*}}{g}\right)^{-1} \alpha_{4}
$$

which can be expressed in terms of the drag coefficient $c_{d}=\left(u_{*} / U_{10}\right)^{1 / 2}$ as

$$
\beta=(2 \pi)^{-1} c_{d}^{-1 / 2} \nu^{-1} \alpha_{4}
$$

where $\nu=f_{m} U_{10} / g$. The formulations of Kahma (1981), Toba (1973) and Donelan et al. (1985) can be related to one another using (1.5).

Theoretical support for the $f^{-4}$ tail for spectra was presented by Kitaigorodskii (1983) using an exact analog of Kolmogoroff's spectrum in a field of weakly nonlinear gravity waves. This Kolmogoroff type equilibrium identifies the energy transfer from lower to 
higher frequencies due to the nonlinear interactions as the limiting process in the growth of the rear face described by a $f^{-4}$ tail. It was also shown that at a higher frequency range it was the wave breaking due to gravitational instabilities that limited the wave growth leading to a $f^{-5}$ tail in this range. Assuming that the three processes wind input, nonlinear wave-wave interactions and wave breaking are equally important in the growth of the rear face of the spectrum, Phillips (1985), using a statistical equilibrium of the three processes, derived a spectral form consistent with Toba's (1972) $f^{-4}$ formulation (1.2). Battjes et al. (1987) reanalyzed the spectra observed during JONSWAP (Hasselmann et al., 1973 ) and showed that a high-frequency tail based on (1.2) is a better fit to the data than one based on (1.1).

\subsection{Equilibrium Range Studies in Finite Depth}

An extension of Phillips (1958) saturation range formulation to finite depth was obtained by Kitaigorodskii et al. (1975) by adopting the wavenumber form of the spectral shape to be valid in any water depth. In shallow water the spectrum then has a limiting form $E(f) \propto f^{-3}$ and evidence of this relation was found in field observations by Knowles (1982) and Goda (1975). Bouws et al. (1985) applied the procedure of Kitaigorodskii et al. (1975) to the entire spectral range and obtained a depth-dependent self-similar spectral shape described by JONSWAP parameters. The analysis was based on measurements from three field programs in finite depth and a statistical basis to quantify the fit of all the spectra to the self-similar shape was proposed.

\subsection{Energy Balance}

The energy transport equation relates the change in energy content of waves with time to the sum of the various input and dissipation source terms. The source terms describing 
wind input, nonlinear wave-wave interactions and wave breaking have been addressed by many authors (Snyder et al., 1981; Hasselmann and Hasselmann, 1981; Komen et al., 1984) and have been employed extensively in wave models in deep water. In finite depth the waves interact with the bottom topography and energy is dissipated due to some of the following physical processes : percolation, depth-induced breaking and bottom friction. Hasselmann and Collins (1968) developed a model for the spectral dissipation of energy due to the interaction of the waves with the bottom boundary-layer. Choosing various formulations for the source terms Bouws and Komen (1983) examined the relative importance of these terms for a growing spectrum in finite depth. A good balance was obtained with an appropriate choice of proportionality coefficients in the formulations of the source terms.

\subsection{Overview}

The field program used in this study is described in Chapter 2 with a summary of the data for the entire duration. In Chapter 3 the relative direction between wind and wave propagation is calculated based on the concept of effective fetch. The rear face of spectra are expressed in terms of the three formulations $(1.1-1.3)$ and the shape parameters are calculated. The quality of fit to the three similarity formulations is expressed on a statistical basis. The spectral parameters are expressed in terms of growth stage and the relations are compared to previous results. Relations between spectral parameters and fetch are calculated. In Chapter 4 various source and dissipation terms are estimated for two cases of measurements of growing wind-seas. The energy balance between various spectral input and dissipation source terms and the relative importance of these sources in maintaining the shape of growing wind-sea spectra in finite depth are discussed. Chapter 5 summarizes the results and conclusions of this study. 


\section{Chapter 2}

\section{Description of the Data Set}

The data analyzed in this study were obtained from a field program conducted by the Canada Centre of Inland Waters (CCIW) and the Great Lakes Environmental Research Laboratory (GLERL) in 1985 in Lake St. Clair to study wave growth and dissipation in finite water depth. Lake St.Clair is a small and shallow lake between Lake Erie and Lake Huron and is connected by the Detroit river and the St.Clair river to these lakes, respectively. The average depth of the lake is about $4 \mathrm{~m}$. The lake has large enough fetches $(40 \mathrm{~km})$ for prevailing west winds to generate waves that are transformed by the interactions with the bottom. Due to geometric restrictions of the lake, multi-modal wave systems (eg. wind seas and swell) are not generated. Meteorological and wave spectral data were collected from mid September to early December 1985.

\subsection{Meteorological Measurements}

Three buoys deployed at locations shown in Figure 2-1 measured the wind speed and direction at a height of $4 \mathrm{~m}$, air and water temparature and relative humidity. The wind direction was also measured using a large vane fixed to the buoy and whose response to wind wave frequencies was largely damped. Data was collected at 10 minute intervals and 


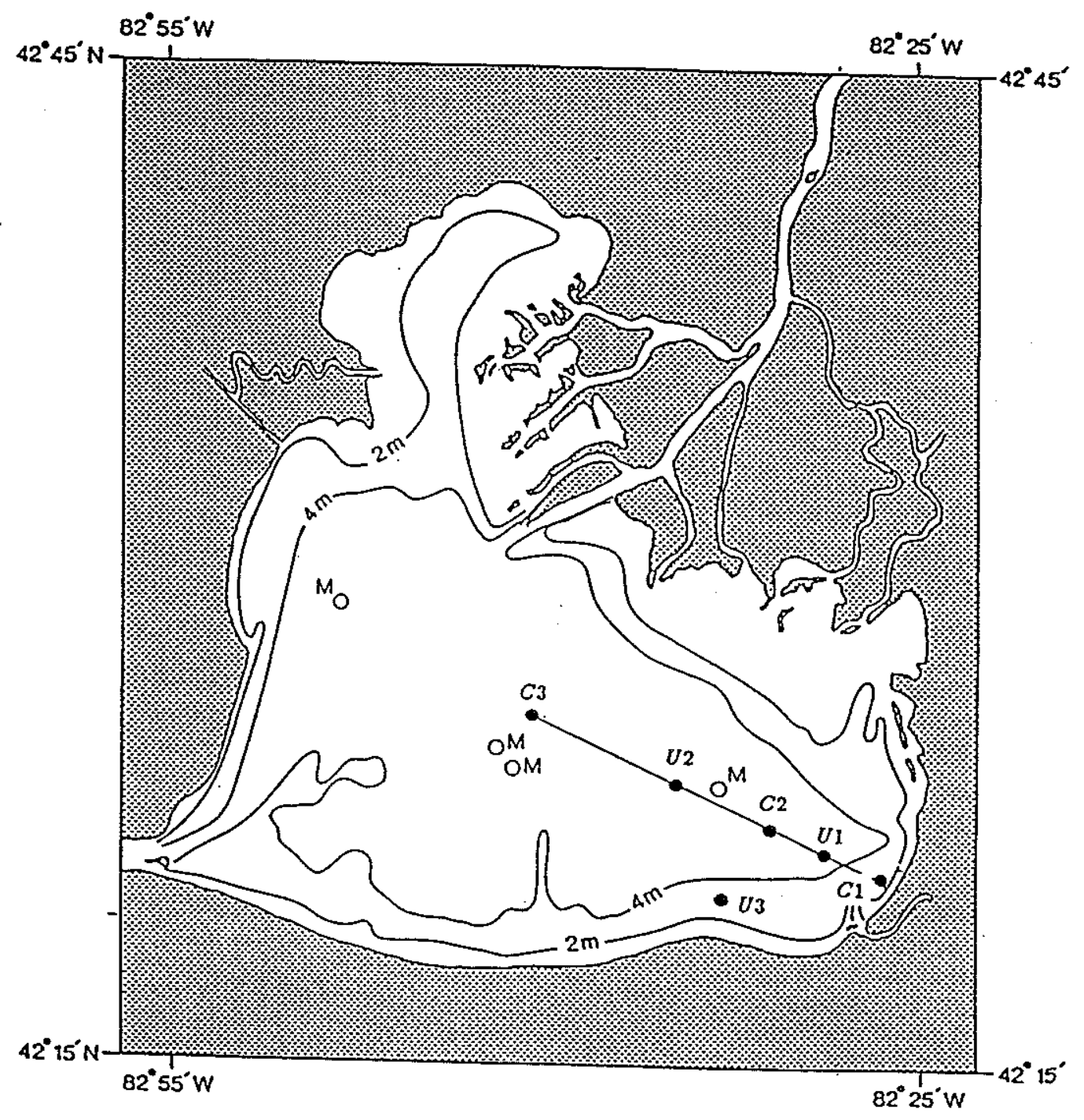

Figure 2-1: Location of measurement stations in Lake St. Clair 
the wind speed at each buoy was obtained as a scalar average and wind direction as a unit vector average of two records (20 minute average). The winds were corrected for stability effects and adjusted for height and then the equivalent $10 \mathrm{~m}$ neutral winds were calculated.

\subsection{Wave Measurements}

Six wave stations located as shown in Figure 2-1 provided wave spectral data. Five of the stations were on a transect 295 degrees from the true north, approximately parallel to the prevailing wind direction. The water depths at the stations ranged from 3.7 to 7.0 meters. Based on expected wave periods in the region a sampling rate of $4 \mathrm{~Hz}$ was selected for the wave data and the record length was set at 4096 samples, or about 17 minutes at $4 \mathrm{~Hz}$. Three of the stations operated by CCIW (marked as $C 1, C 2$ and $C 3$ in Fig. 2-1) consisted of a triangular array of capacitance wave gages and a Seadata recorder. These stations recorded samples beginning every even-numbered hour when the wind speed exceeded a preset threshold value $(6-8 \mathrm{~m} / \mathrm{s})$. They provided wave direction estimates along with the energy spectrum for periods of large wave heights. Due to a problem in resolving the wave directions observed at the Canadian towers the direction information is not used in this analysis. The other three stations provided by GLERL (marked as $U 1, U 2$ and $U 3$ in Fig. 2-1) consisted of a single Zwarts transmission wave staff and radio transmitter. These stations transmitted continously to a shore station and an hourly averaged spectral data were recorded every hour.

\subsection{Summary of Data}

Time series plots of measured and computed spectral parameters at each tower for entire duration of the field program are presented in Figs. 2-2 to 2-13. In Figs. 2-2 to 2-7 the 
measured variables, vector wind, wind speed $(U)$, significant wave height $\left(H_{s}\right)$, spectral peak frequency $\left(f_{m}\right)$, air and sea temparature, water level (GLERL stations only) are plotted. The wind speed $(U)$ used here is the neutral wind speed at $10 \mathrm{~m}$ elevation and is an average of the measurements from the three meteorological buoys. In Figs. 2-8 to 2-13 the following computed non-dimensional spectral parameters

$$
\begin{aligned}
\omega_{h} & \equiv 2 \pi f_{m}(h / g)^{1 / 2}=\text { dimensionless depth } \\
\kappa_{m} & \equiv \frac{k_{m} U^{2}}{g}=\text { dimensionless wavenumber } \\
\nu & \equiv \frac{f_{m} U}{g}=\text { dimensionless frequency } \\
\frac{U}{c} & \equiv \frac{U}{g / \omega_{m} \tanh k_{m} h}=\text { relative wind input }
\end{aligned}
$$

are presented for the entire duration, where $k_{m}$ and $c$ are the wave number and the phase speed corresponding to the peak frequency $\left(f_{m}\right), \omega_{m}=2 \pi f_{m}$ and $h$ is the water depth. 

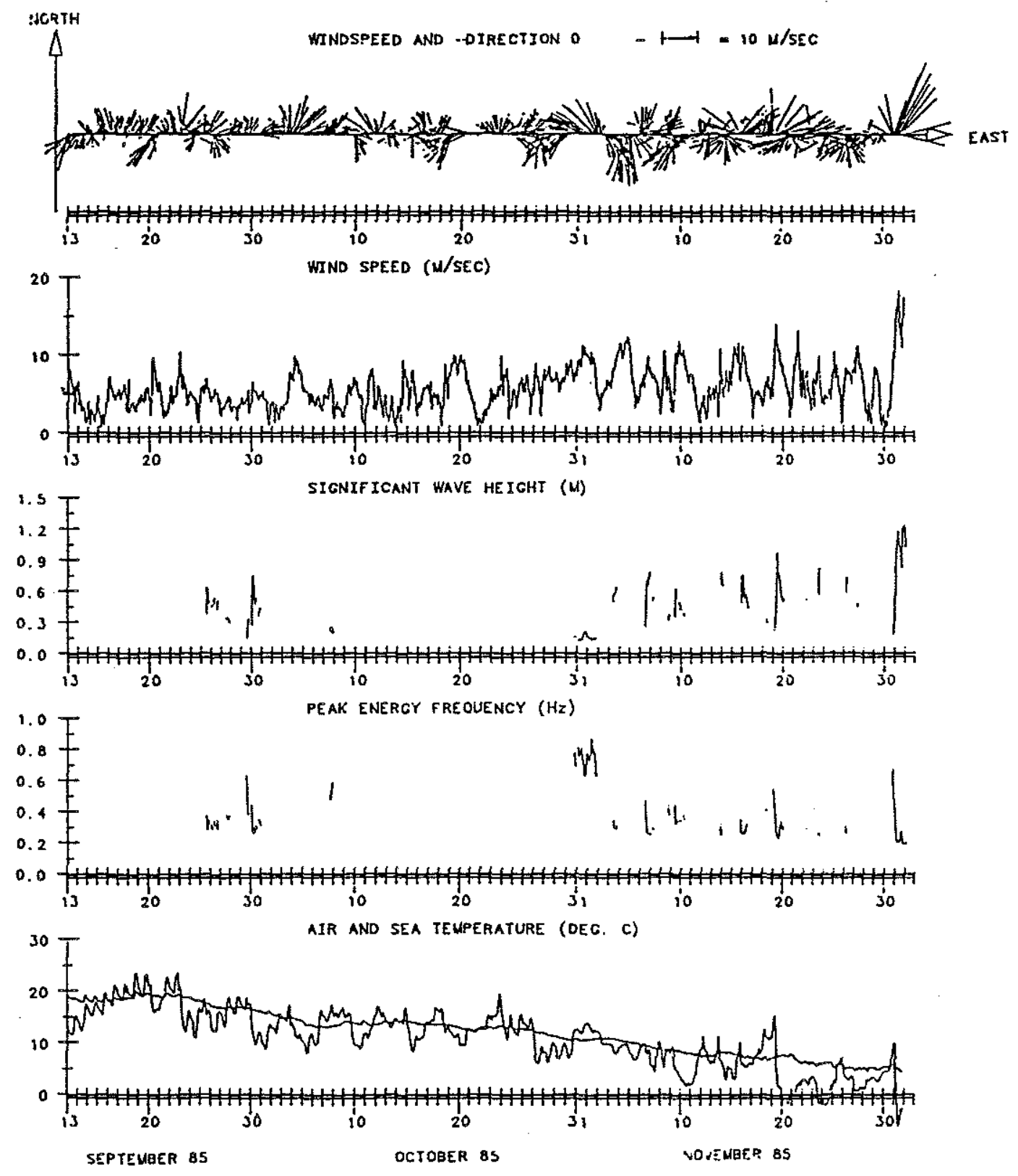

Figure 2-2: Time series of measurements at tower $C 1$ 

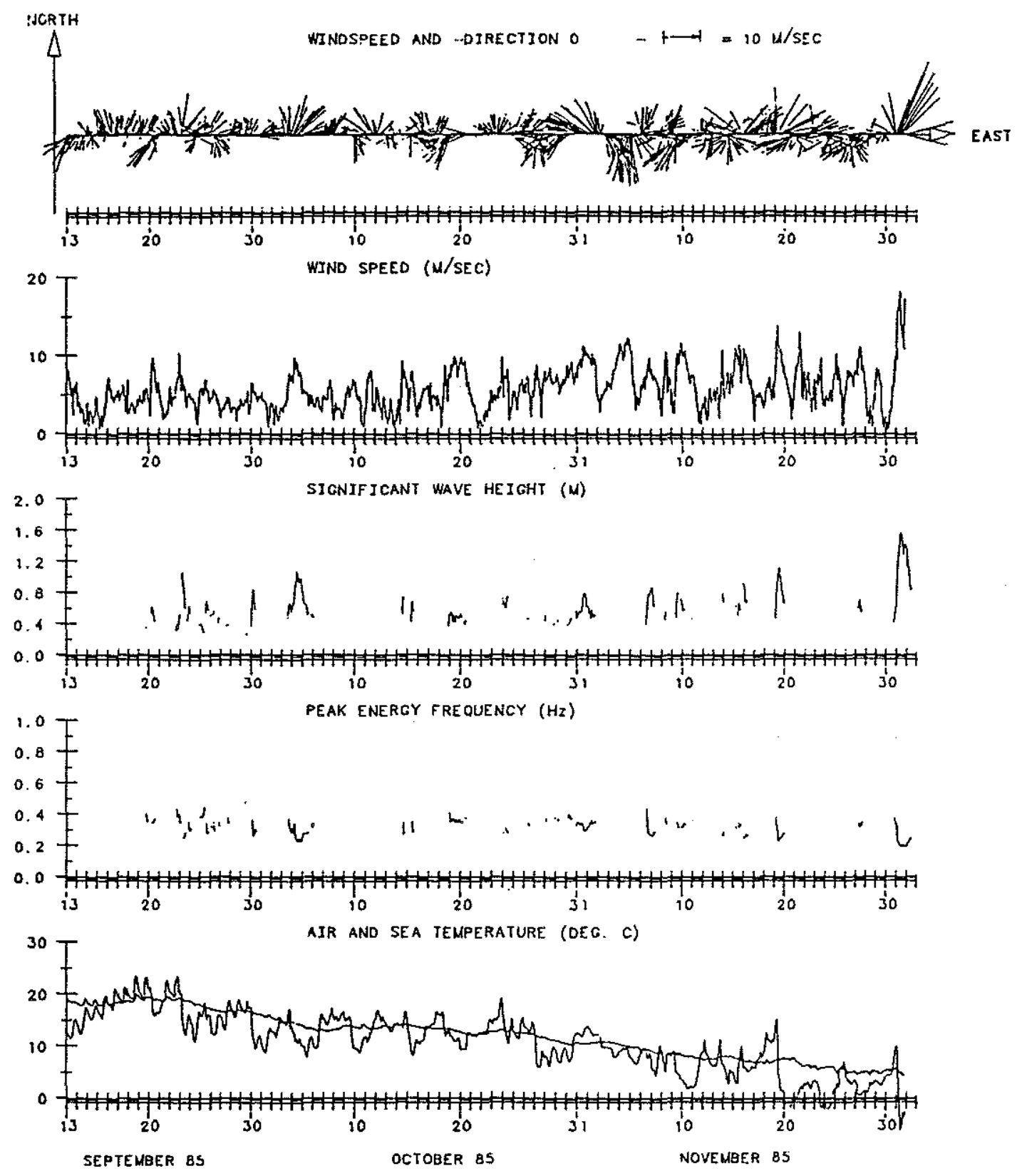

Figure 2-3: Time series of measurements at tower $C 2$ 


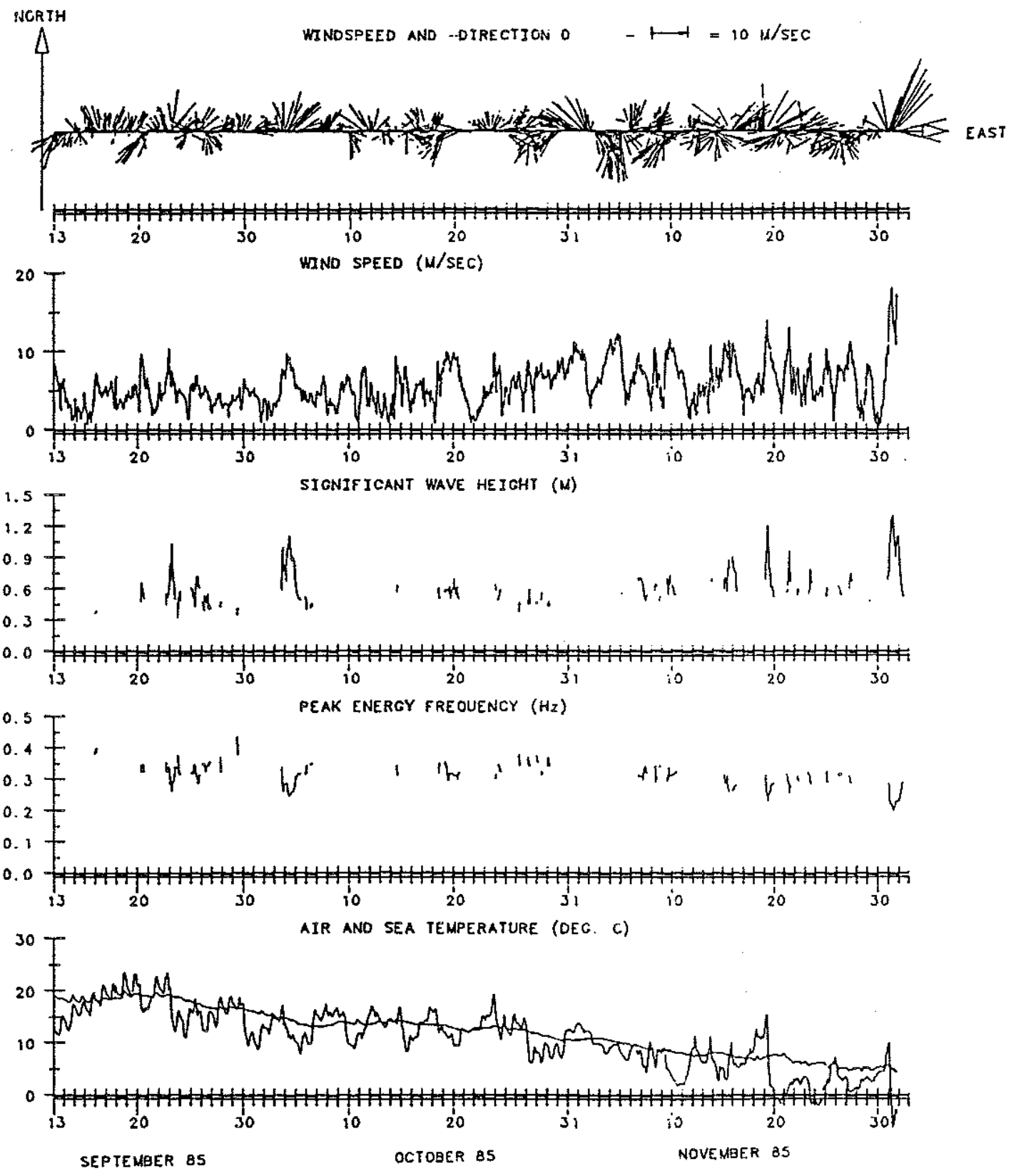

Figure 2-4: Time series of measurements at tower $C 3$ 


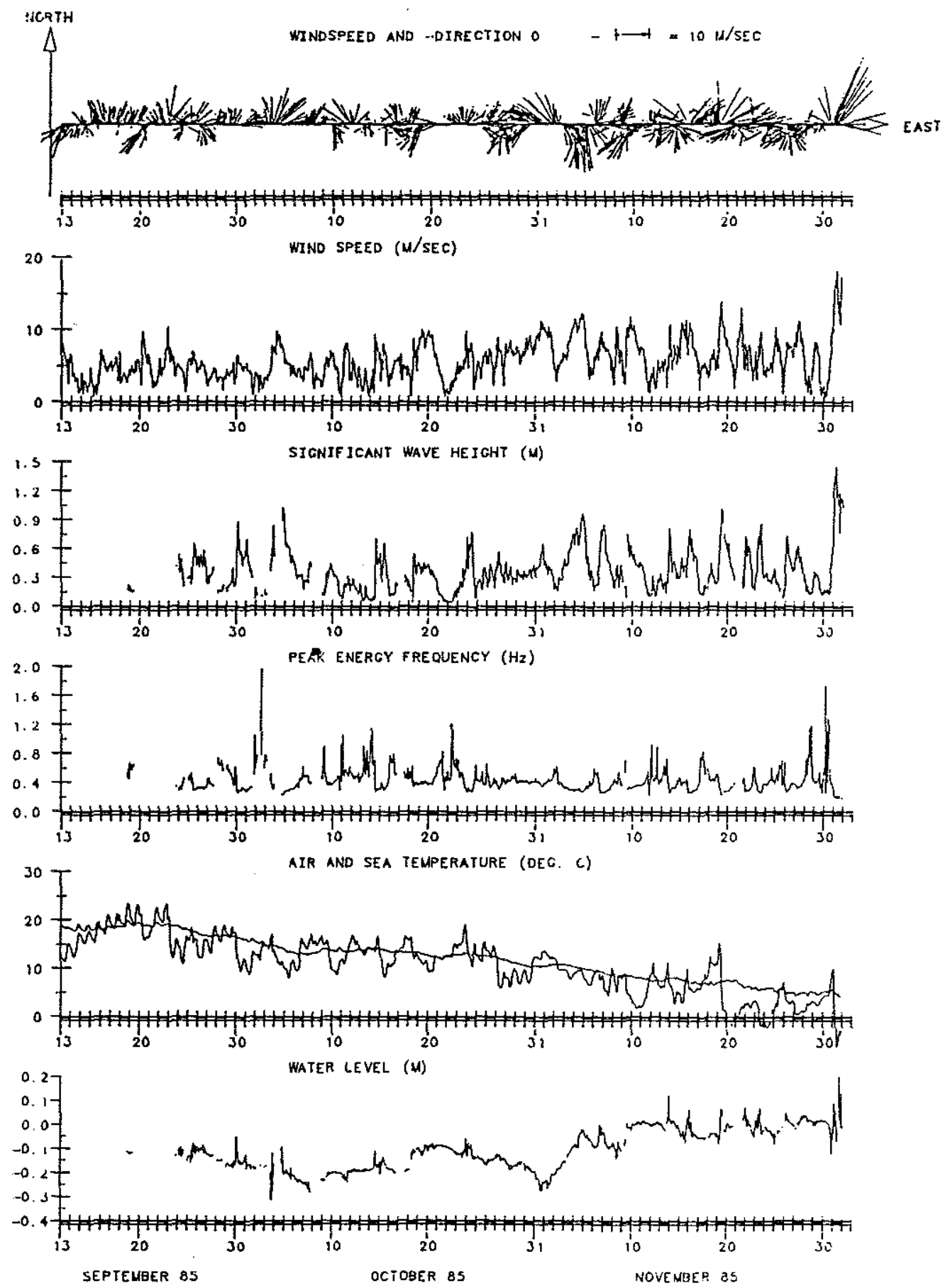

Figure 2-5: Time series of measurements at tower $U 1$ 


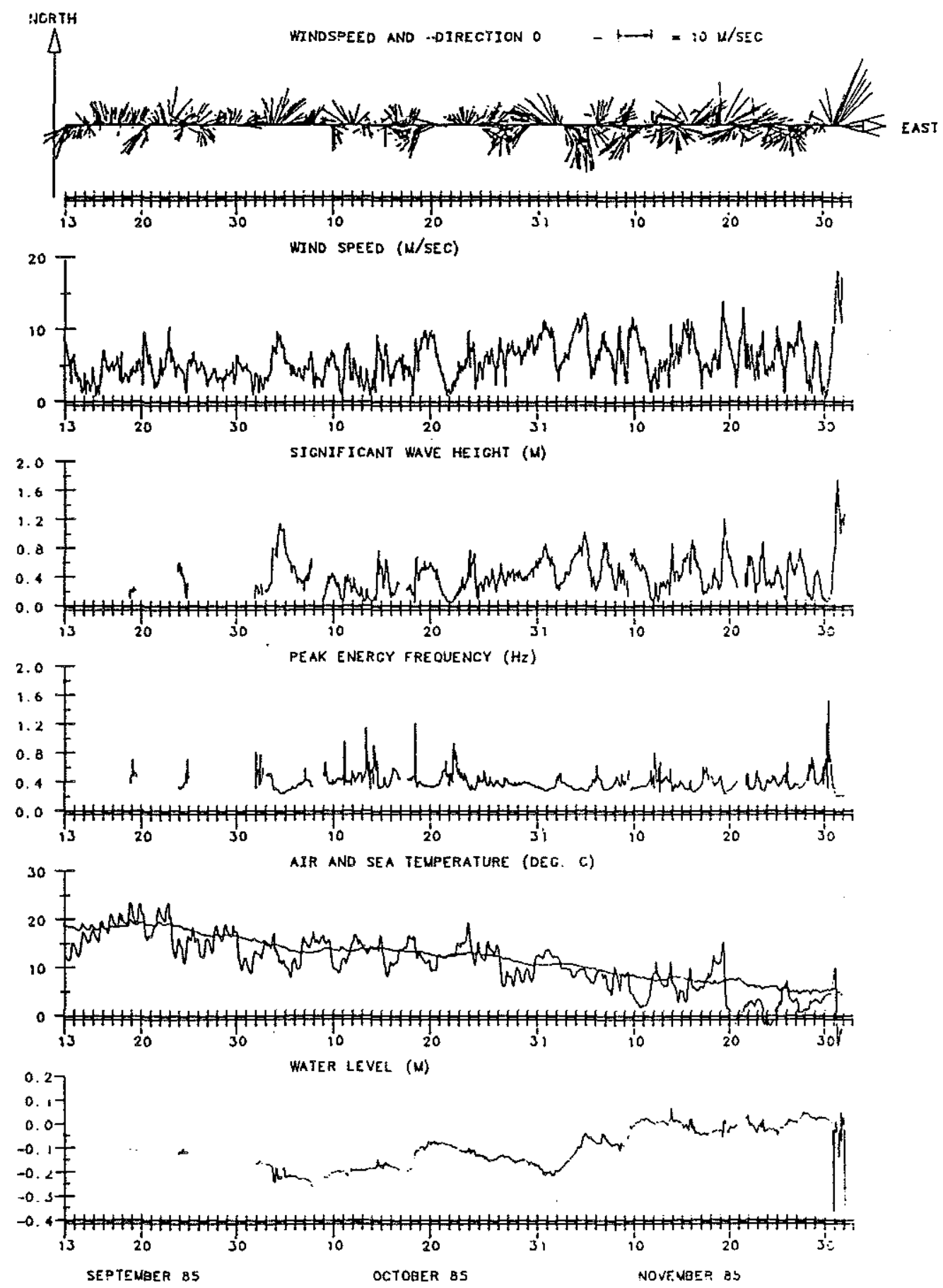

Figure 2-6: Time series of measurements at tower $U 2$ 


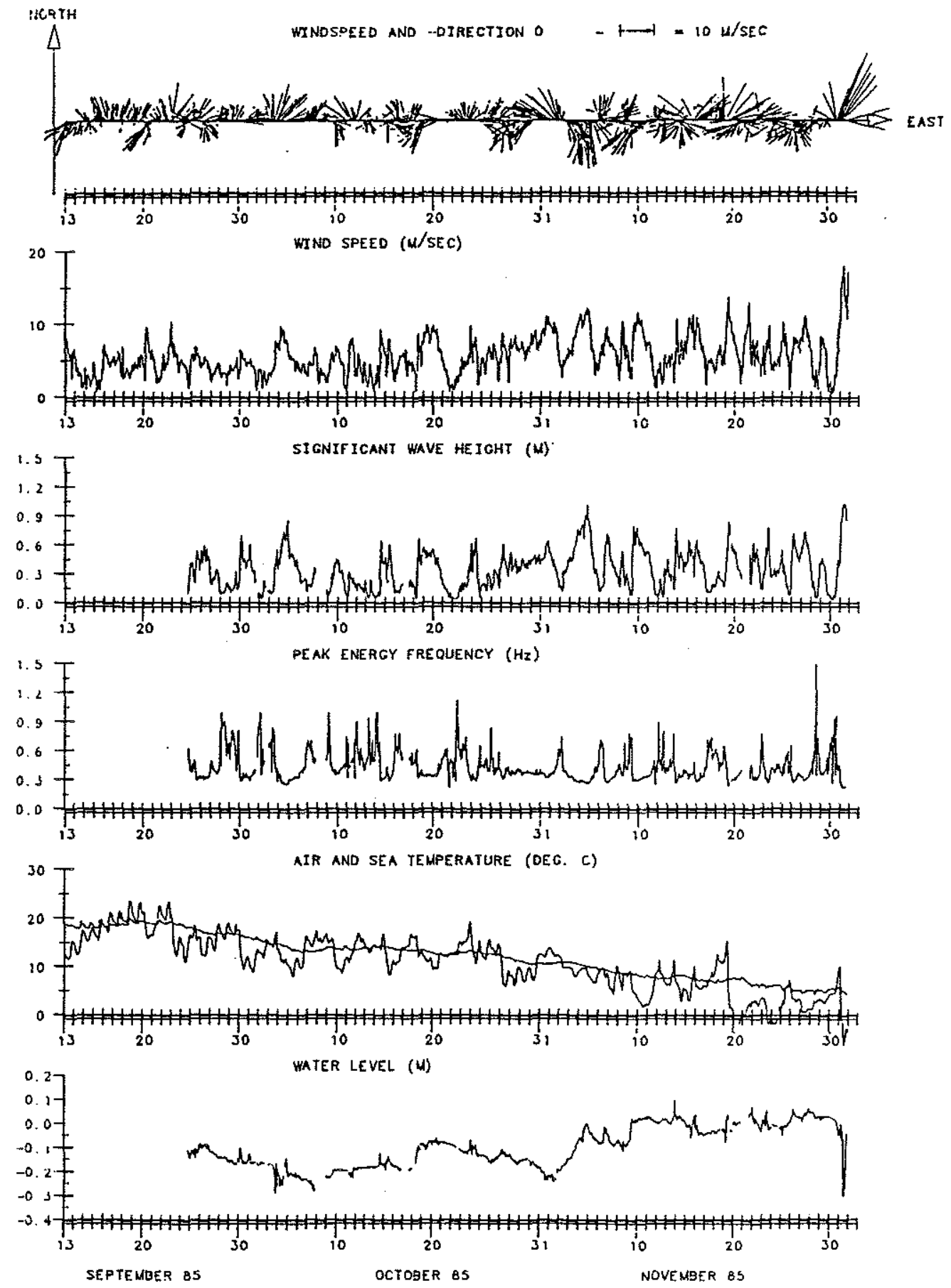

Figurę 2-7: Time series of measurements at tower $U 3$ 

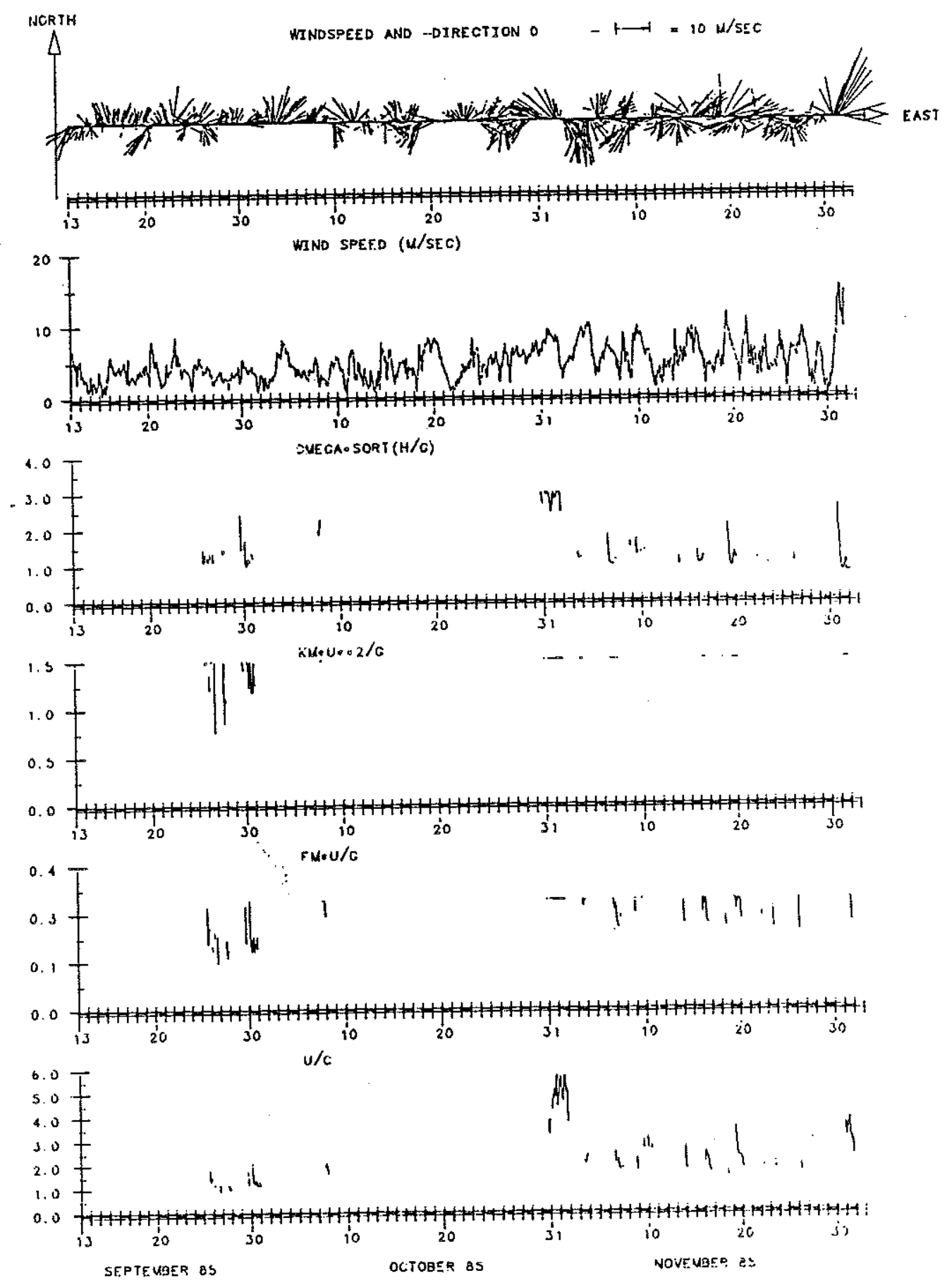

Figure 2-8: Time series of spectral parameters at tower $C 1$ 


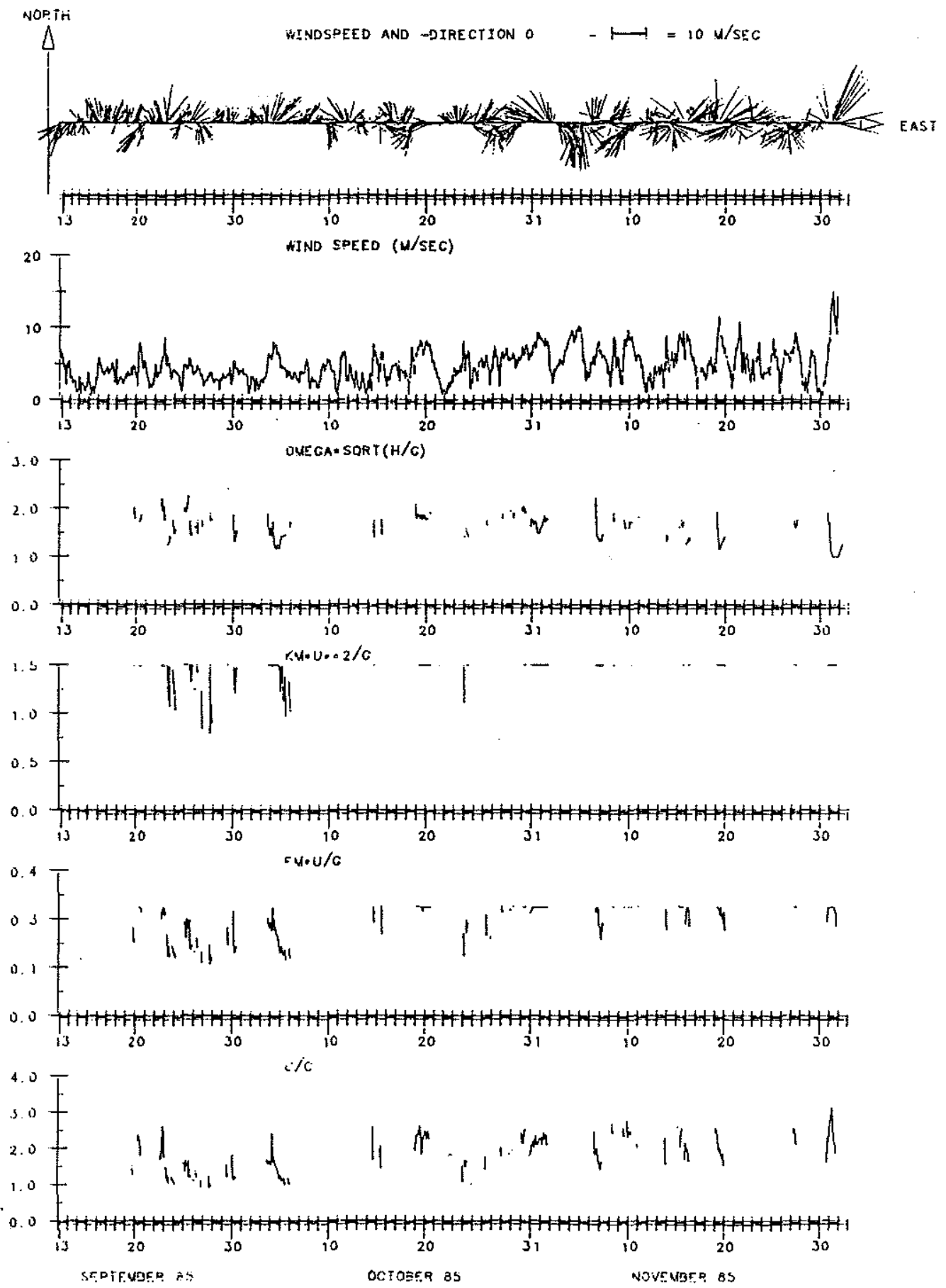

Figure 2-9: Time series of spectral parameters at tower $C 2$ 


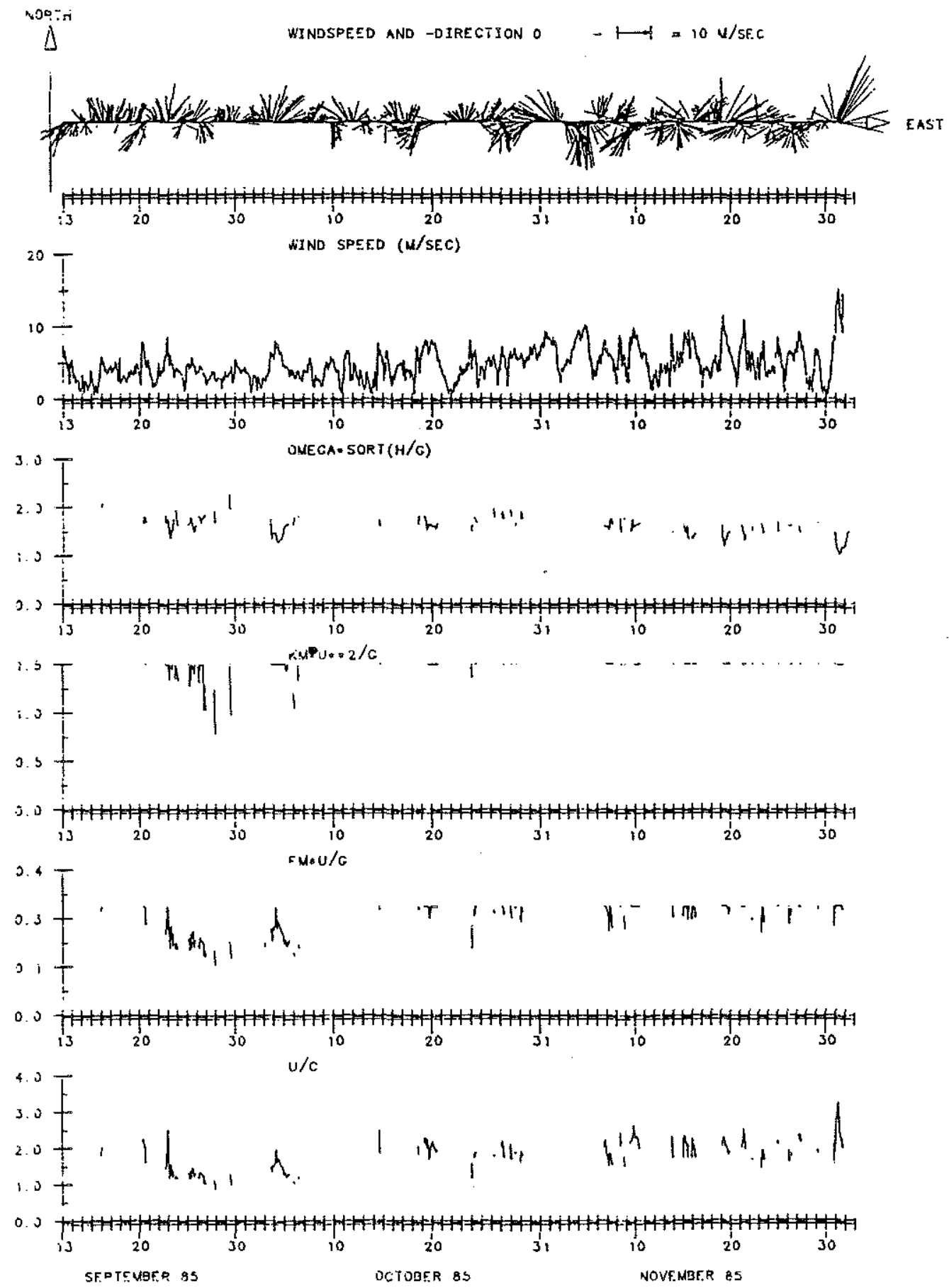

Figure 2-10: Time series of spectral parameters at tower $C 3$ 


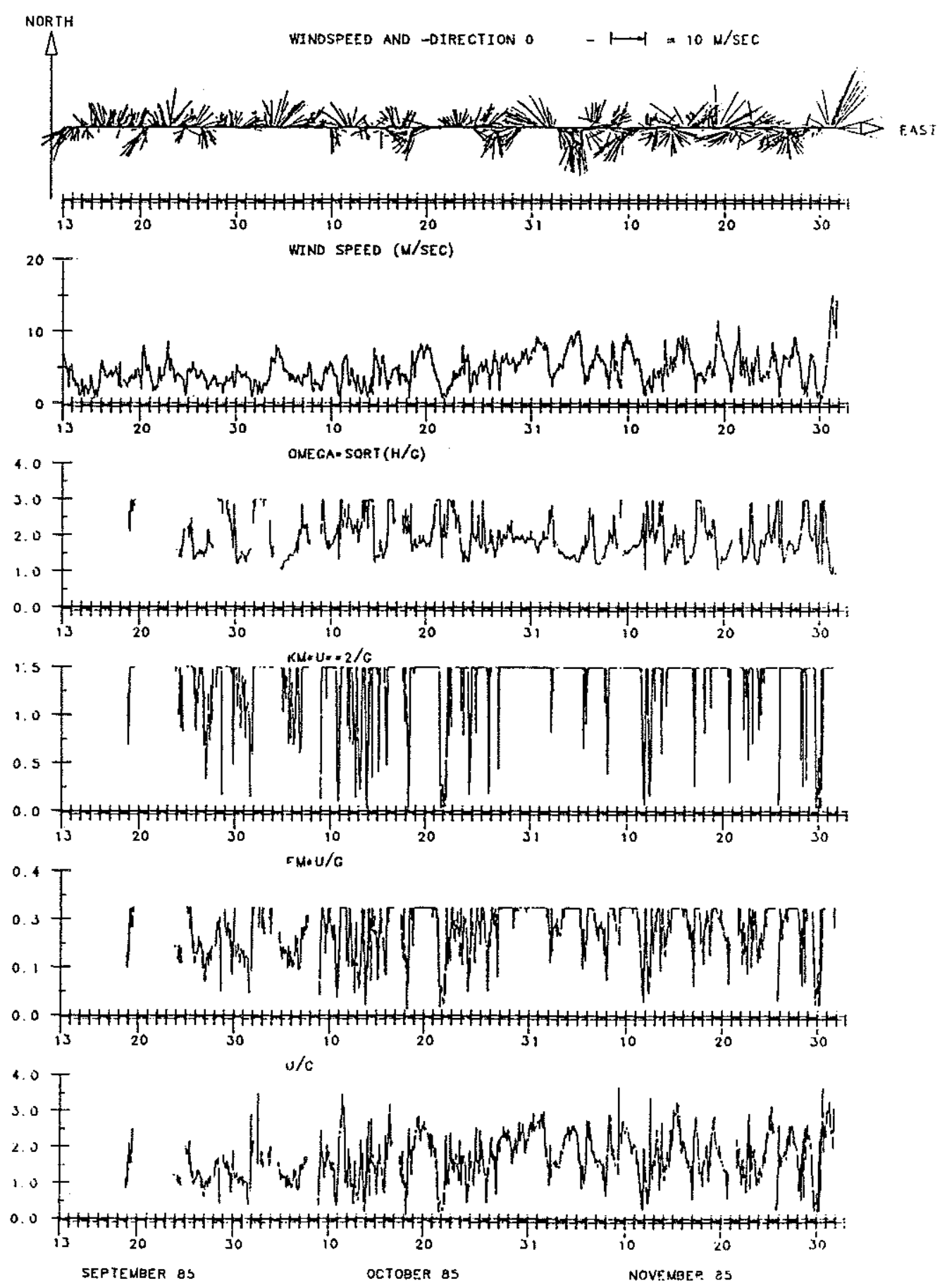

Figure 2-11: Time series of spectral parameters at tower $U 1$ 


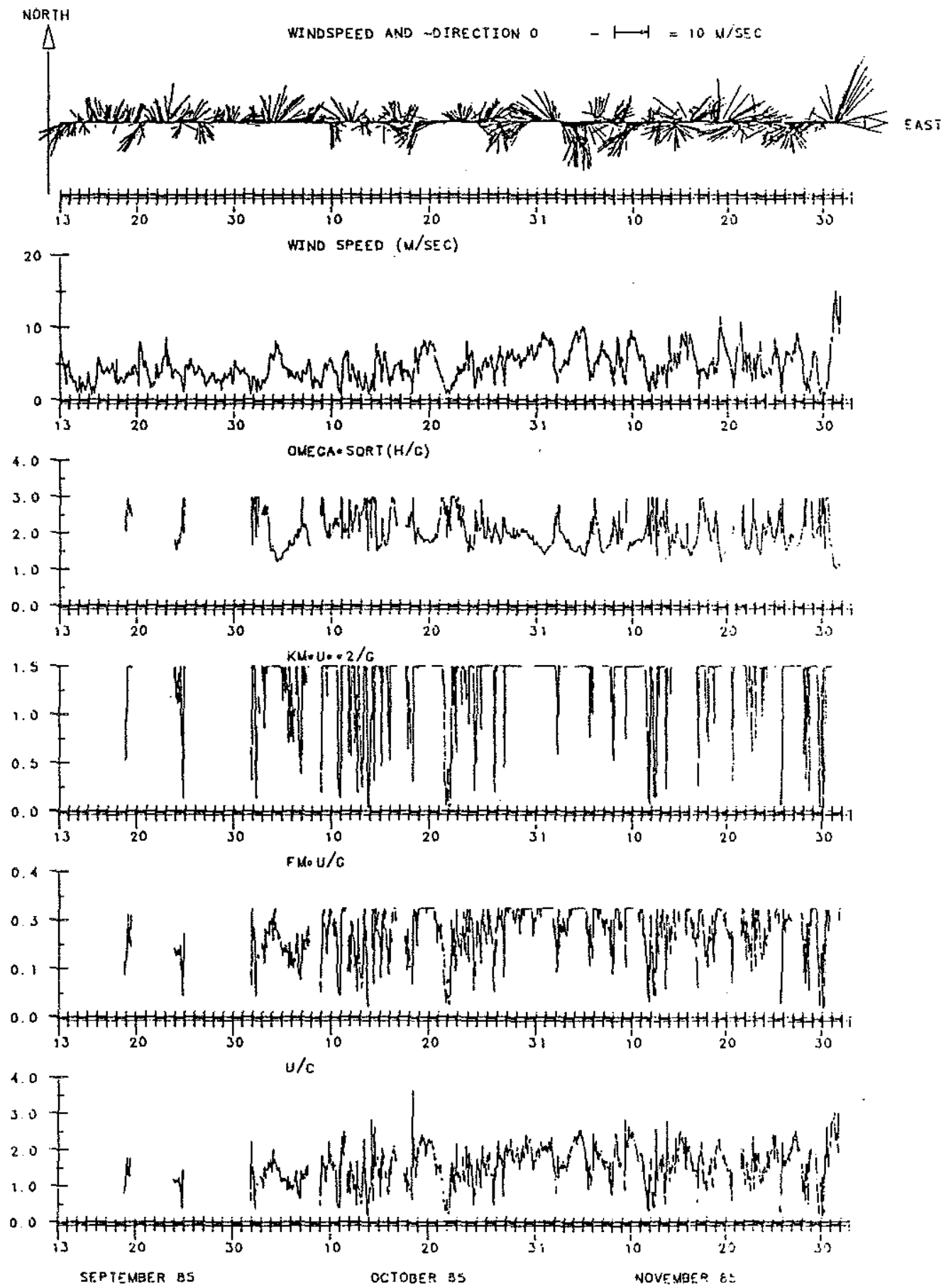

Figure 2-12: Time series of spectral parameters at tower $U 2$ 


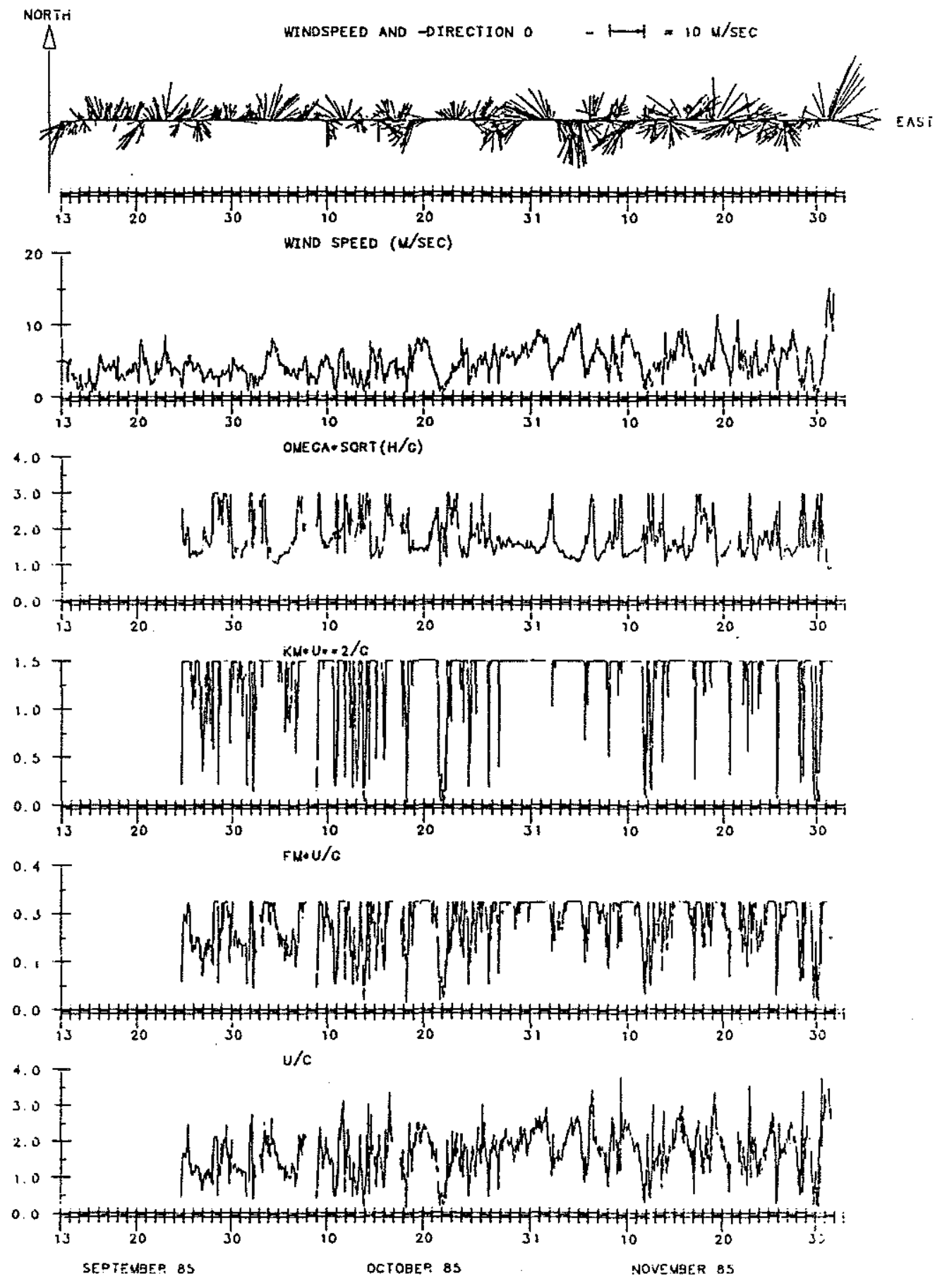

Figure 2-13: Time series of spectral parameters at tower $U 3$ 


\section{Chapter 3}

\section{Similarity Relations}

\subsection{Peak Wave Direction}

Recent meaurements (Donelan et al., 1985) have shown that for fetch-limited seas the wave and wind directions are not coincident. Knowles (1982) had observed from wave measurements in a fetch restricted basin that when the growth of wave energy and peak frequency were expressed in terms of a simple geometric fetch it was observed the long fetch range relation and the short fetch range relation had the same slope but were displaced from one another. An effective fetch formulation

$$
X_{e}=\frac{\Sigma X_{i} \cos \theta_{i}}{\Sigma \cos \theta_{i}}
$$

where $X_{i}$ are the radial length and $\theta_{i}$ are angles (less than $90^{\circ}$ ) on either side of the wind axis was used to recalculate the relation. This formulation gave a single growth relation which was close to the relation for the short fetch ranges indicating that the displacement was due to the overestimation of the long range fetches in the simple fetch formulation. For an enclosed body of water with highly restricted fetch it was shown by Donelan (1980) that the prevailing wave direction depends on the effective fetch. Using the functional dependence of wave period on fetch and component wind speed given by Donelan et al. (1985) it was 
shown that the effective fetch is the geometric fetch $X_{i}$ that maximizes $\cos \left(\theta_{i}\right) X_{i}^{426}$. As the wind direction is not a constant, an average value of $X_{i}$ over a $30^{\circ}$ window $\left( \pm 15^{\circ}\right)$ was used for $X_{i}$ instead of the direct line distance from the shore to the tower (Donelan, 1980) for better results.

The complete data set was analyzed using Donelan's (1980) procedure and the wave directions $\left(\theta_{e}\right)$ at each tower was calculated based on effective fetches. In Figure 3-1 the predicted wave directions for all CCIW towers are plotted versus the observed wind directions. The directions for the different stations are marked with different symbols $(\triangle-C 1 ; x-C 2$ and $\circ-C 3)$. It is clear that the propagation directions of the waves can be as much as $60^{\circ}$ off the wind direction when the wind directions are near $50^{\circ}, 250^{\circ}$ and $320^{\circ}$ especially in the case of $\mathrm{C} 1$. The rapidly changing geometric shape of the lake near these regions give rise to the relative angle between wind and wave direction. The pronounced differences observed at tower $\mathrm{C} 1$ conform to the variations in the lake geometry. Similar differences in wind and wave directions have been observed by Schwab et al. (1984) for wind-sea measurements conducted in Lake Erie.

\subsection{Equilibrium Range}

Wind-wave spectra have a sharp cut-off below the peak frequencies and most of the energy is contained in the region above the peak frequency. The three different spectral forms given in (1.1 - 1.3) express the the rear face of the spectrum in terms of a functional dependence on frequency and an equilibrium parameter. By analysing the fit of the data to the three formulations the proper functional form of the energy spectrum is identified.

The equilibrium parameters $\alpha_{4}, \beta$ and $\alpha_{5}$ are calculated from the energy spectrum in the equilibrium range. The equilibrium range is defined as the range of frequencies satisfying $f_{1}<f<f_{2}$, where $f_{1}=1.5 f_{m}$ and $f_{2}=3.5 f_{m}$. The measured spectral density values 


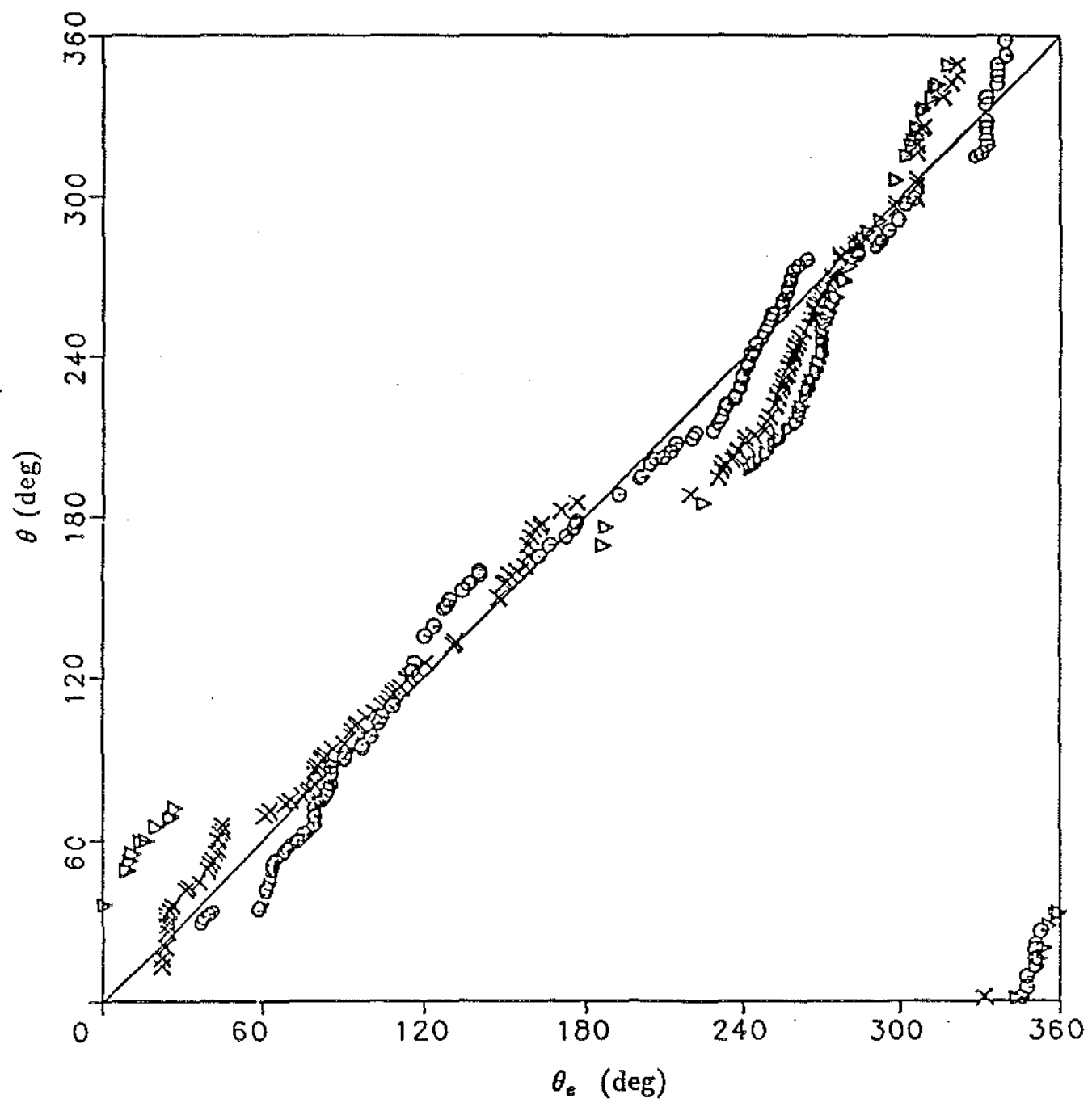

Figure 3-1: Observed wind and predicted wave directions at CCIW towers $(\triangle-C 1 ;+-C 2 ; 0-C 3)$. 
in this range are multiplied with the appropriate functional forms (see eqs. $1.1-1.3$ ) and averaged over the range to obtain the equilibrium parameters. In Fig. 3-2 the values of the equilibrium parameters are plotted as a function of the equilibrium range frequencies and it is clear that a simple average is a good approximation for the parameters.

To test the fit of the data to the three formulations a statistical quality control is derived based on Bouws et al. (1985). Assuming that the spectral form in wave-number space is universal (Kitaigorodskii et al., 1975), relations between the total energy and the equilibrium parameters are derived for the three different formulations. From the data set relations between the total energy and the equilibrium parameters are calculated and compared to the theoretical relations. For deep water conditions the energy spectrum in wave-number space $F(k)$ can be found from the energy spectrum in frequency space by equating the total energy of the waves

$$
\int E(f) d f=\int F(k) d k
$$

where $k$ is the wave number. For (1.1) the energy spectrum $F(k)$ (using the deep water dispersion relation $\omega^{2}=g k$ ) can be written as

$$
F(k)=\frac{\alpha_{5}}{2} k^{-3} \psi\left(k, f_{m}, H\right)
$$

where $\psi$ is the spectral shape function. The shape function $\psi$ goes rapidly to zero below $k_{m}$ (the wave number corresponding to the frequency $f_{m}$ ) and modifies the higher wave number region mildly. Assuming the shape of the energy spectrum $F(k)$ to be universal the energy of the waves $\tilde{E}$ in any depth is given by

$$
\tilde{E}=\int_{0}^{\infty} F(k) d k=\frac{\alpha_{5}}{2} \int_{0}^{\infty} k^{-3} \psi\left(k, f_{m}, H\right) d k
$$

Neglecting the contribution of $\psi$, a first approximation of the energy is given by

$$
\tilde{E}=\frac{\alpha_{5}}{2} \int_{k_{m}}^{\infty} k^{-3} d k
$$



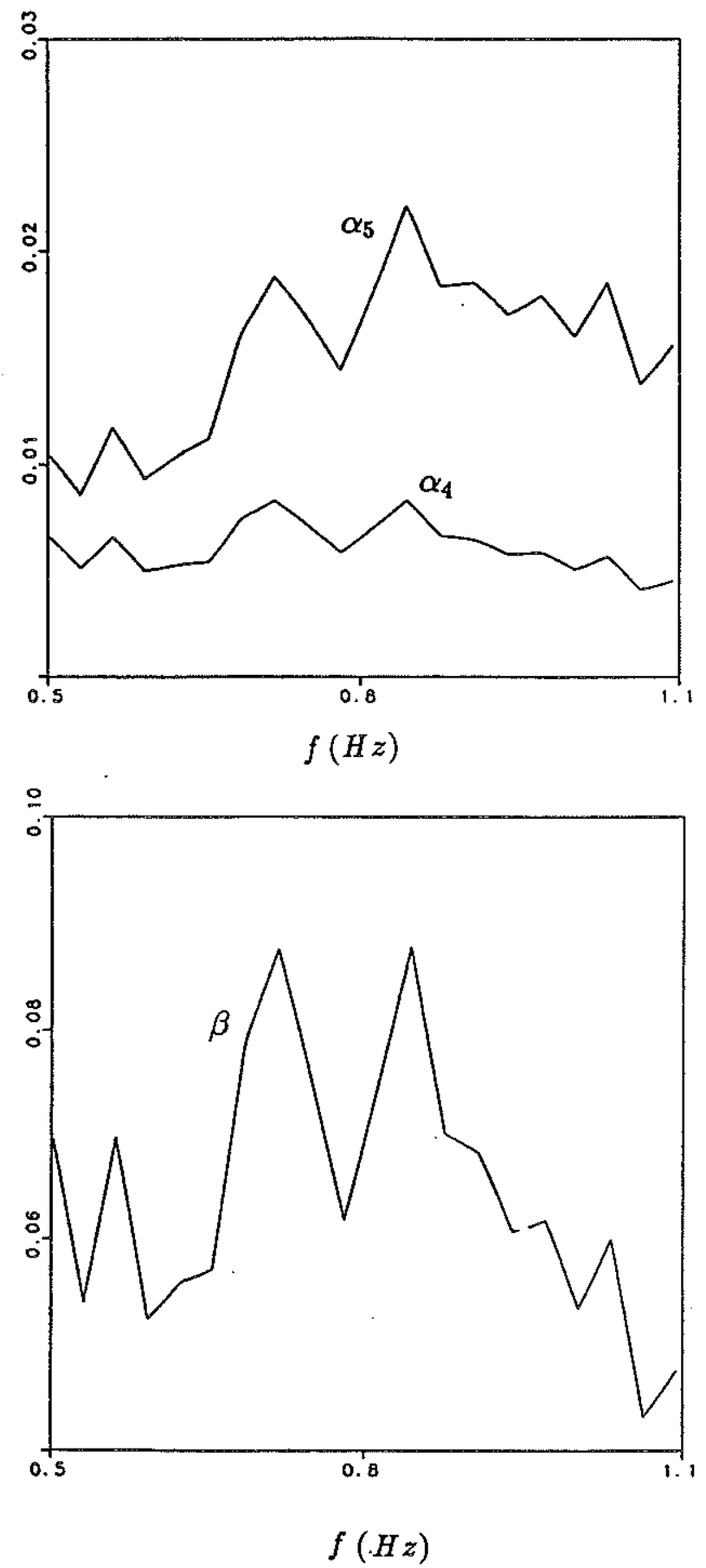

Figure 3-2: Equilibrium parameters as function of frequency 
Integration leads to the energy relation

$$
\tilde{E}=\frac{\alpha_{5}}{4} k_{m}^{-2}
$$

and in nondimensional form ( 3.5 ) becomes

$$
\epsilon=\frac{\alpha_{5}}{4} \kappa_{m}^{-2}
$$

where

$$
\epsilon=\tilde{E} g^{2} / U^{4}
$$

is dimensionless energy and

$$
\kappa_{m}=k_{m} U^{2} / g
$$

is the dimensionless wave number. Similarly, the energy relations corresponding to (1.2) and (1.3) are given by

$$
\epsilon=\frac{u_{*}}{3 U} \beta \kappa_{m}^{-1.5}
$$

and

$$
\epsilon=\frac{\alpha_{4}}{3} \kappa_{m}^{-2}
$$

where $u_{*} / U=c_{d}^{1 / 2}$. The relation ( 3.5) was given for deep water by Huang et al. (1980).

Using the values of the equilibrium parameters $\alpha_{4}, \beta$ and $\alpha_{5}$ and the values of $\epsilon$ and $\kappa_{m}$ calculated for each observation a linear regression analysis based on logarithmic scales is performed. The fit of the regression line is expressed with the coefficients $r$ and $A$

$$
\ln \epsilon=r \ln (B)+\ln A
$$

where $B=\alpha_{5} \kappa_{m}^{-2}$ for (3.5), $B=\frac{u_{*}}{U} \beta \kappa_{m}^{-1.5}$ for ( 3.9) and $B=\alpha_{4} \kappa_{m}^{-2}$ for (3.10). In Figure 3-3 to 3-6 $\epsilon$ is plotted versus $\alpha_{4} \kappa_{m}^{-2}$ for the entire data set, for $U=|U|$, on a $\log -\log$ scale for various ranges of the dimensionless depth $\omega_{h}$. The regression lines for the different $\omega_{h}$ 
ranges are

$$
\begin{array}{ll}
\epsilon=0.635\left(\alpha_{4} \kappa_{m}^{-2}\right)^{1.00} & \text { for } \omega_{h}<1 \\
\epsilon=0.202\left(\alpha_{4} \kappa_{m}^{-2}\right)^{.847} & \text { for } 1.0 \leq \omega_{h}<1.3 \\
\epsilon=0.182\left(\alpha_{4} \kappa_{m}^{-2}\right)^{.835} & \text { for } 1.3 \leq \omega_{h}<1.8 \\
\epsilon=0.152\left(\alpha_{4} \kappa_{m}^{-2}\right)^{.813} & \text { for } \omega_{h} \geq 1.8 .
\end{array}
$$

The theoretical relation (3.10) is compared to the regression lines (3.12 to 3.15) in Figures 3-3 to 3-6 and the applicability of the formulation (1.3) can be quantified by the closeness of the regression parameters $r$ and $A$ to 1 and 0.333 respectively. Except for the range $\omega_{h}<1$ ( Fig 3-3) the regression lines show similar characterstics indicating no significant dependence on depth. In Fig. 3-3 for $\omega_{h}<1$ the regression line has the same slope as the theoretical relation but is displaced from it. There is a large scatter in the data for the range $\omega_{h}>1.8$ and this may be due to the low wave heights and wind speed values. The GLERL stations recorded measurements ditring all wind conditions and may have been contaminated by disturbances due to other sources, like boats, when the wind speeds were low. A lower limit on the measured wind speed and wave heights was set and all data points that fall below this limit were excluded and new regression parameters were calculated. Based on the local conditions the limits chosen were $U=3 \mathrm{~m} / \mathrm{s}$ and $H_{s}=0.25 \mathrm{~m}$. In Table 3.1 the new regression relations are compared to the relations obtained for the entire data set. It is seen that when the contamination by low wind speeds and wave height is absent the regression relations are closer to the theoretical relation (3.10) than the regression relation of the entire data set.

The heuristic lower limit on wave number $k_{m}$ defining a fully developed sea state is given by the deep-water expression

$$
\kappa_{m}=\frac{k_{m} U^{2}}{g}=0.667
$$

which corresponds to the Pierson-Moskowitz frequency $f_{P M}=0.13 \mathrm{~g} / U$ which is the lower 


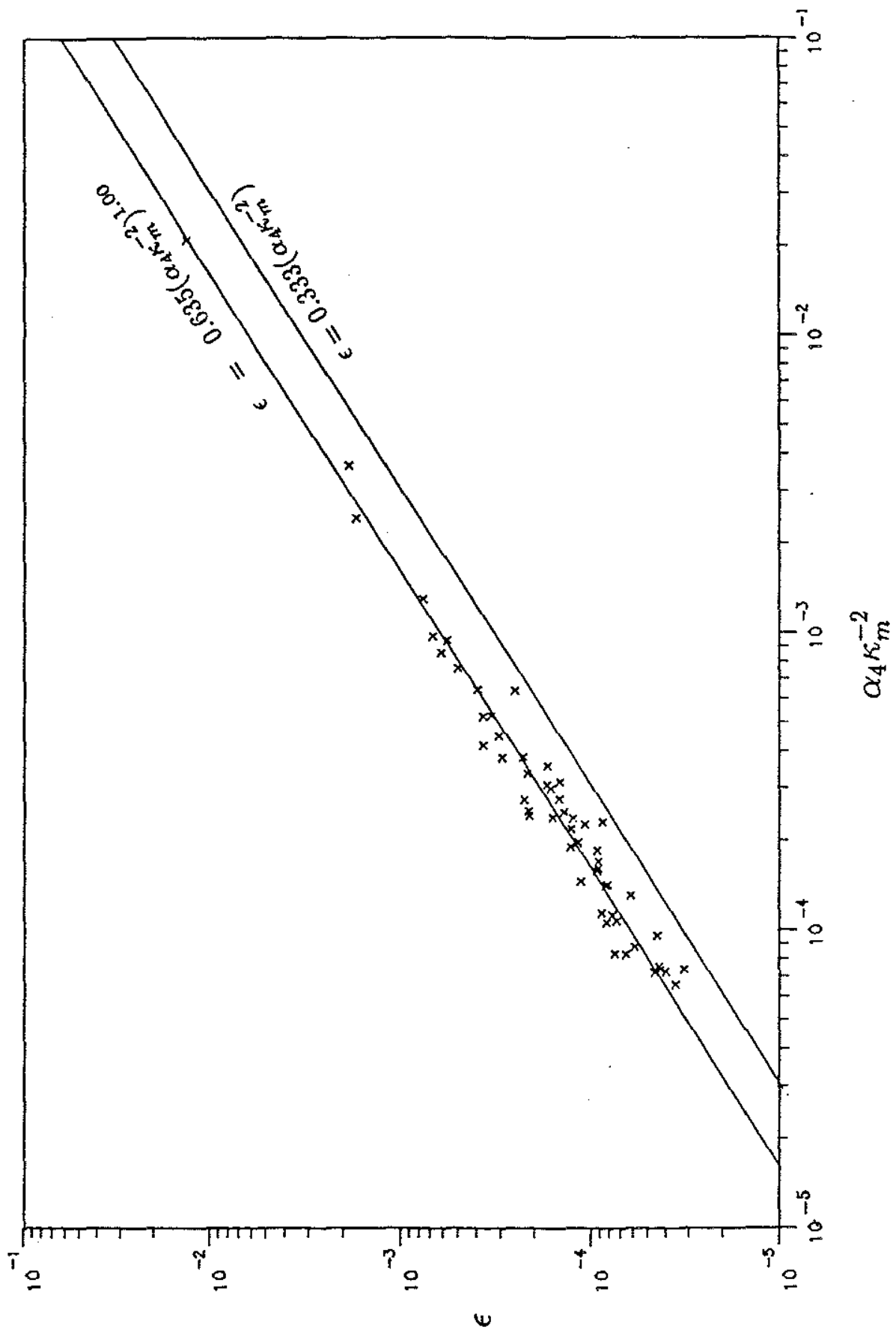

Figure 3-3: Dimensionless energy $\epsilon$ versus scaling parameters $\alpha_{4} \kappa_{m}^{-2}$ for $\omega_{h}<$ 1.0. The two lines represent the regression relation (3.12) and the theoretical relation (3.10). 


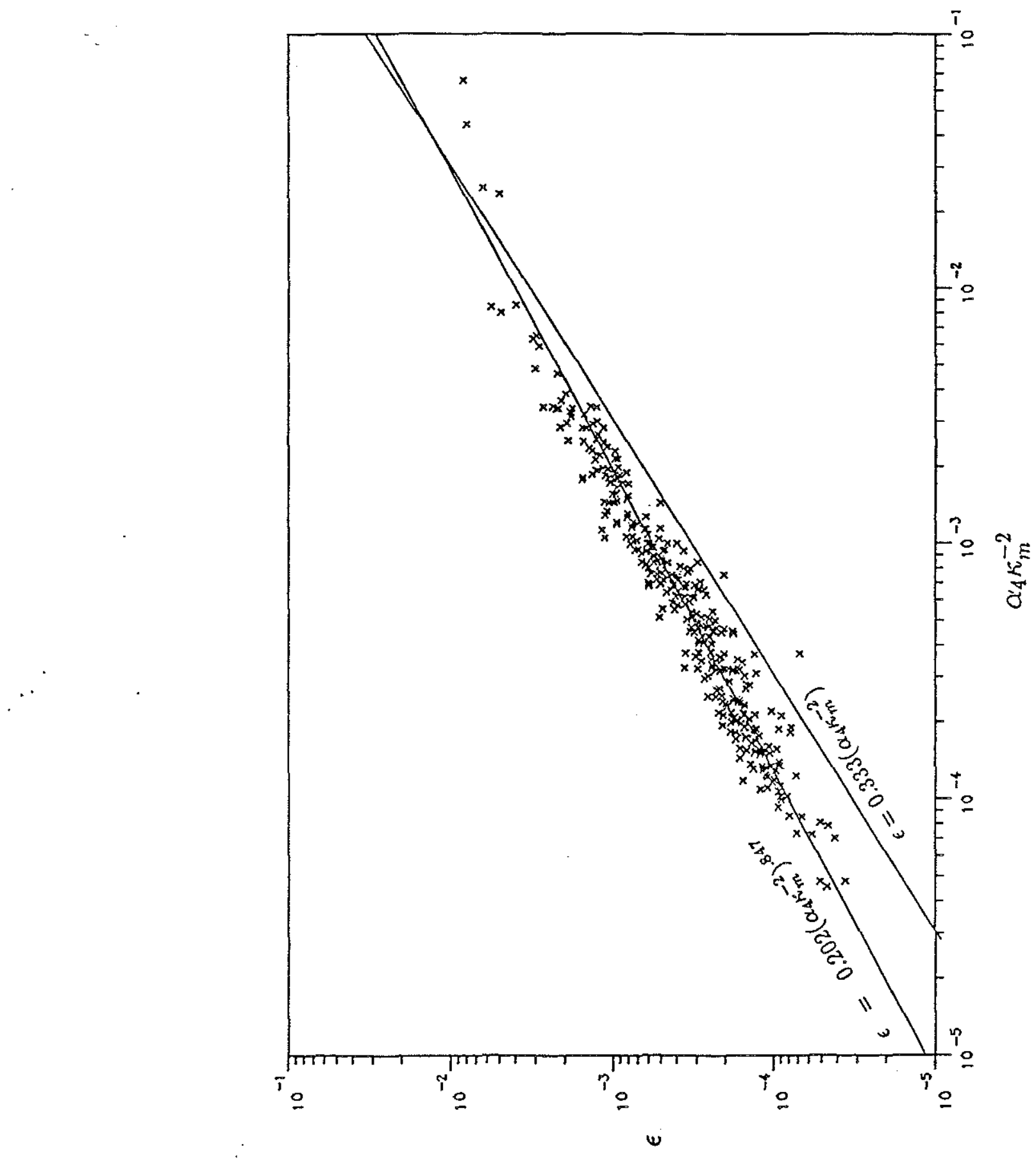

Figure 3-4: Same as Figure 3-3, but $1.0 \leq \omega_{h}<1.3$

38 


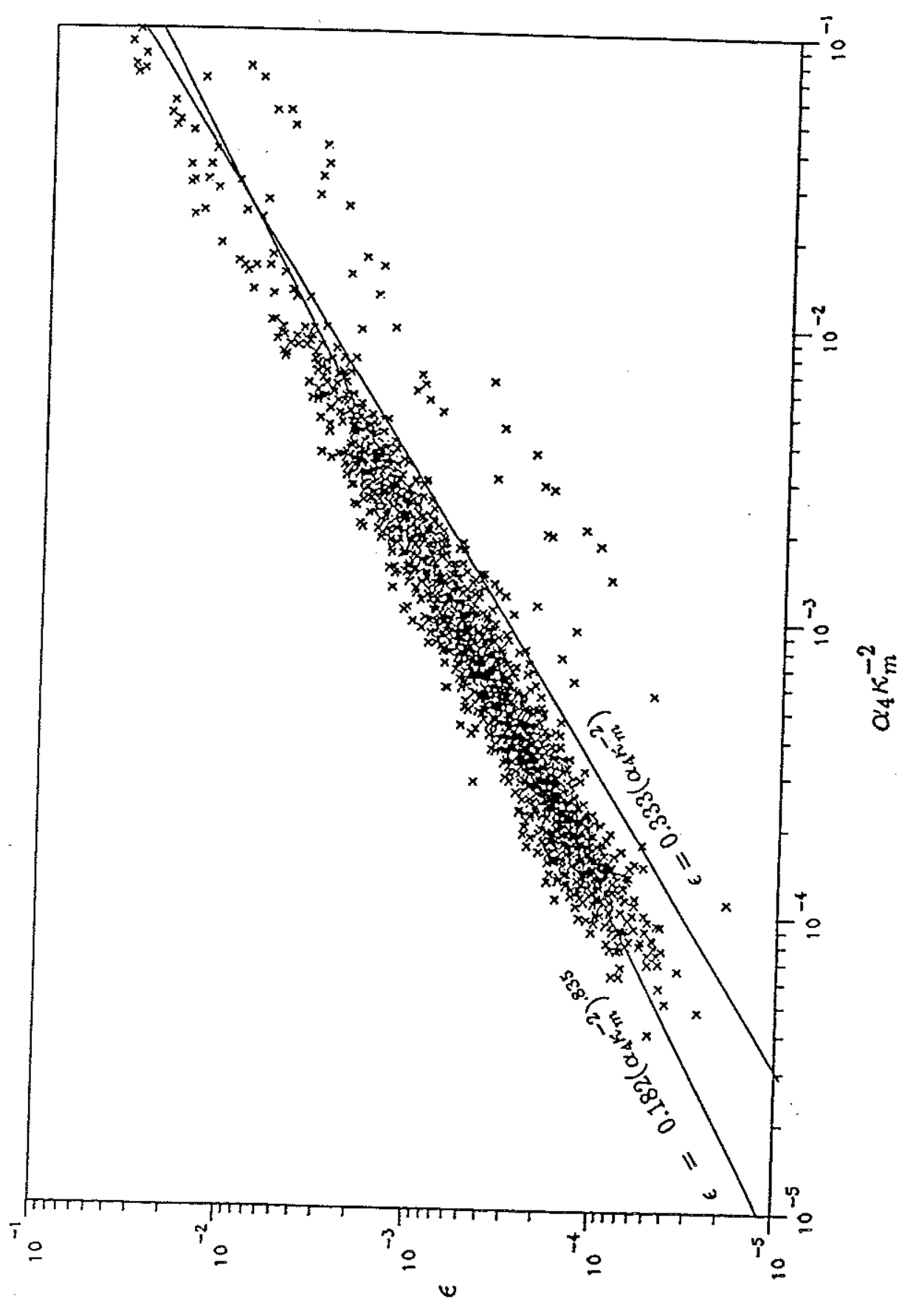

Figure 3-5: Same as Figure 3-3, but $1.3 \leq \omega_{h}<1.8$ 


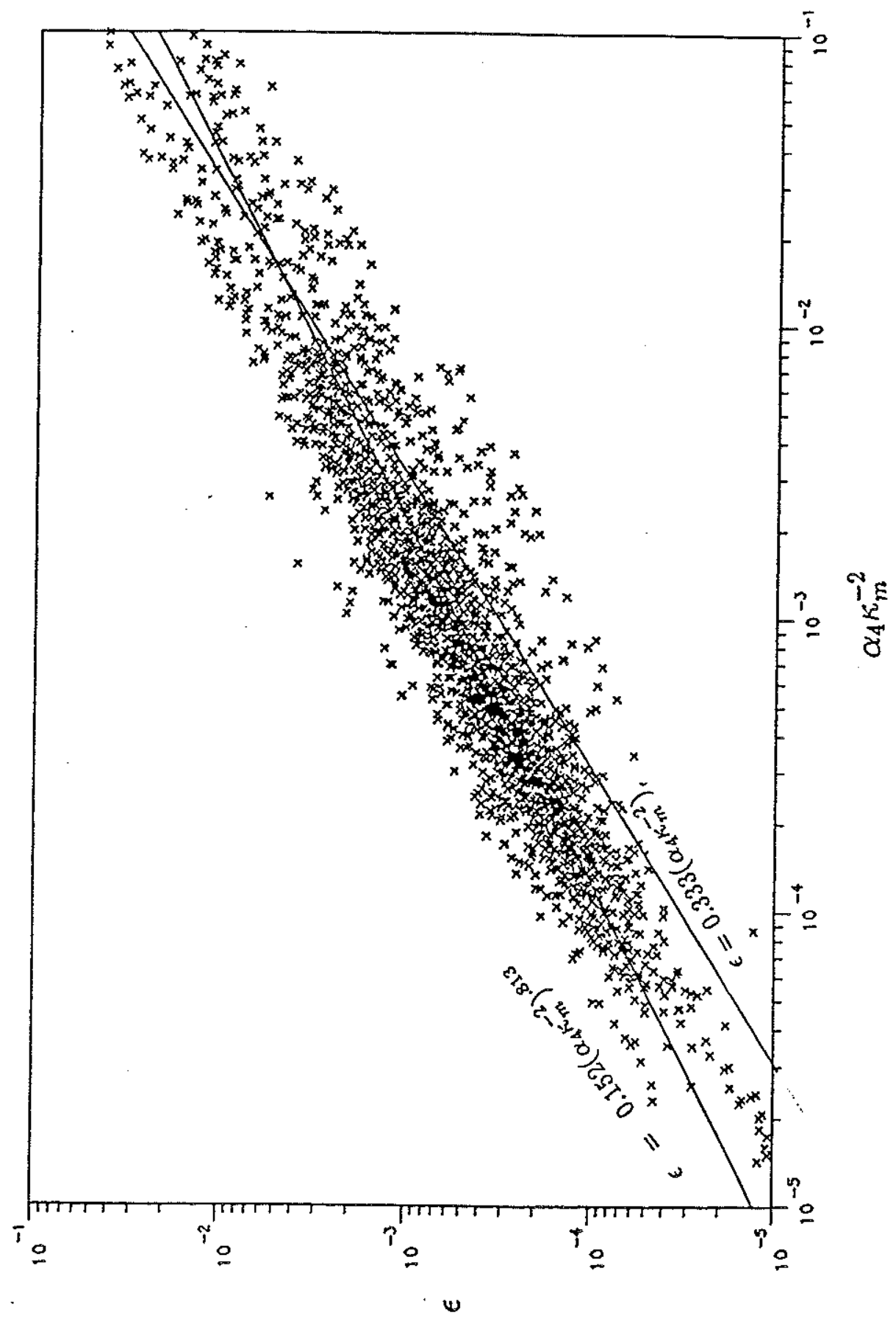

Figure 3-6: Same as Figure 3-3, but $\omega_{h} \geq 1.8$ 


\begin{tabular}{|c|c|c|c|c|}
\hline Range & \multicolumn{2}{|c|}{ Entire Data } & \multicolumn{2}{c|}{ with $U>3 \& H_{s}>0.25$} \\
\hline & No. & Relation & No. & Relation \\
\hline$\omega_{h}<1$ & 56 & $\epsilon=0.635\left(\alpha_{4} \kappa_{m}^{-2}\right)^{1.00}$ & 55 & $\epsilon=0.624\left(\alpha_{4} \kappa_{m}^{-2}\right)^{1.00}$ \\
\hline $1 \leq \omega_{h}<1.3$ & 373 & $\epsilon=0.202\left(\alpha_{4} \kappa_{m}^{-2}\right)^{.847}$ & 368 & $\epsilon=0.246\left(\alpha_{4} \kappa_{m}^{-2}\right)^{.872}$ \\
\hline $1.3 \leq \omega_{h}<1.8$ & 2143 & $\epsilon=0.182\left(\alpha_{4} \kappa_{m}^{-2}\right)^{.835}$ & 2012 & $\epsilon=0.250\left(\alpha_{4} \kappa_{m}^{-2}\right)^{.864}$ \\
\hline$\omega_{h} \geq 1.8$ & 2468 & $\epsilon=0.152\left(\alpha_{4} \kappa_{m}^{-2}\right)^{.813}$ & 1111 & $\epsilon=0.212\left(\alpha_{4} \kappa_{m}^{-2}\right)^{.844}$ \\
\hline all & 5040 & $\epsilon=0.164\left(\alpha_{4} \kappa_{m}^{-2}\right)^{.822}$ & 3546 & $\epsilon=0.241\left(\alpha_{4} \kappa_{m}^{-2}\right)^{.867}$ \\
\hline
\end{tabular}

Table 3.1: Comparison of regression relations for new data set

limit of migration for the spectral peak. Sea states with $\kappa_{m}<0.667$ are no longer under the influence of winds, i.e. they are decoupled, and therefore do not adhere to the similarity hypothesis. From the analysis of the new data set (with $U>3 \mathrm{~m} / \mathrm{s}$ and $H_{s}>0.25 \mathrm{~m}$ ) it is seen that almost all the data points satisfy $\kappa_{m}>0.667$ and represent growing sea states under the influence of wind. Henceforth all the analysis is done using the new data set.

In Figure 3-7 $\epsilon$ is plotted versus $\alpha_{4} \kappa_{m}^{-2}$ for $U=|\mathbf{U}|$, on a $\log -\log$ scale. In the figure different symbols are used to classify the data points based on the value of the nondimensional depth $\omega_{h} .\left(x-\omega_{h}<1 ; \square-1.0 \leq \omega_{h}<1.3 ;+-1.3 \leq \omega_{h}<1.8 ; \triangle-\omega_{h} \geq 1.8\right)$. The regression relation for all the $\omega_{h}$ ranges is given by

$$
\epsilon=0.241\left(\alpha_{4} \kappa_{m}^{-2}\right)^{.867}
$$

In the figure the regression relation (3.17) and the theoretical relation (3.10) are shown for comparison. In Figures 3-8 and 3-9 $\epsilon$ versus $\beta$ and $\epsilon$ versus $\alpha_{5}$ are plotted for the spectral formulations (1.2) and (1.1). The regression relations for all the $\omega_{h}$ ranges,

$$
\epsilon=0.242\left(\frac{u_{*}}{U} \beta \kappa_{m}^{-1.5}\right)^{.869}
$$




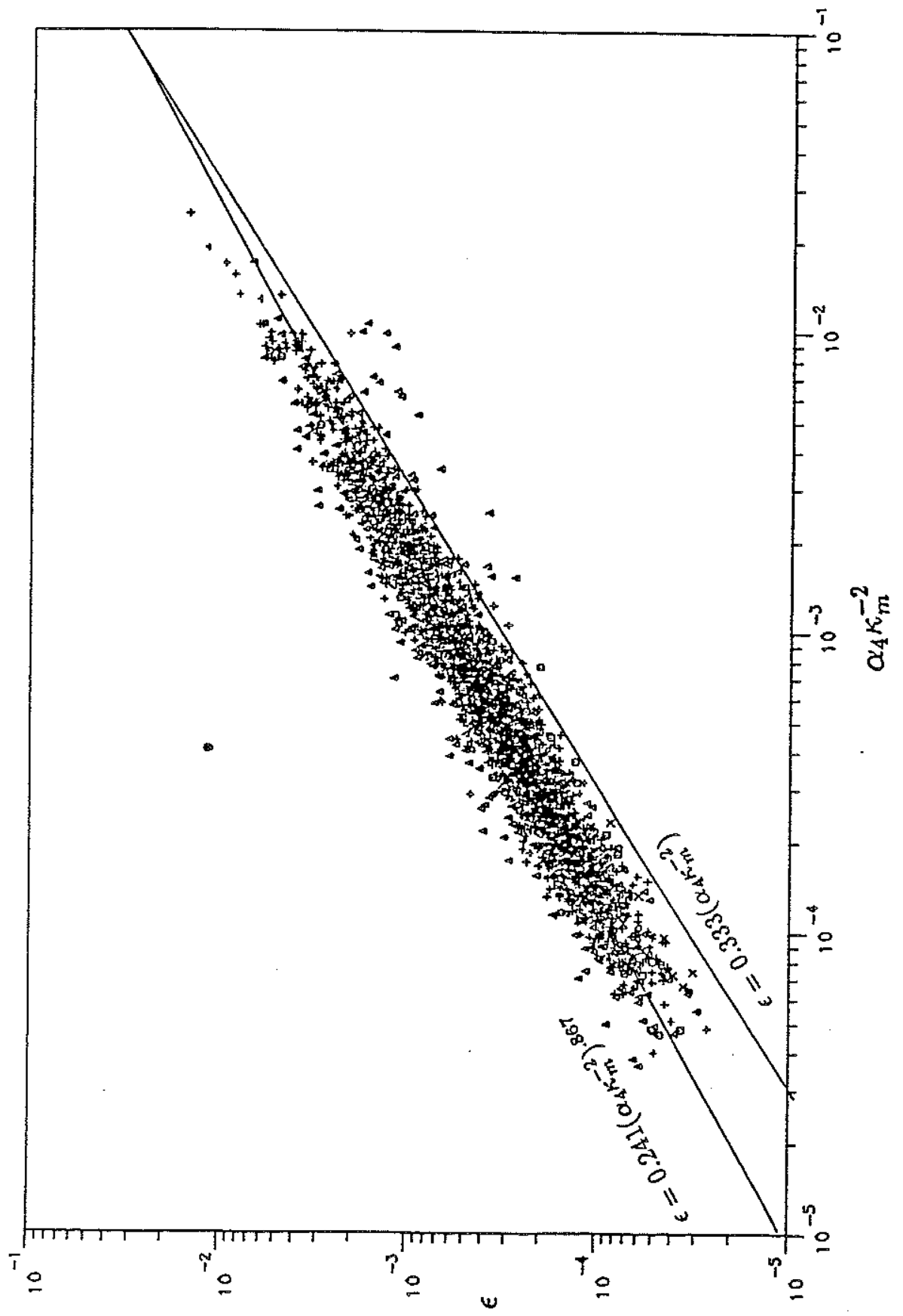

Figure 3-7: Dimensionless energy $\epsilon$ versus scaling parameters $\alpha_{4} \kappa_{m}^{-2}$ for the entire data set. The two lines represent the regression relation (3.17) and the theoretical relation (3.10). The different symbols represent classification based on dimensionless depth $\omega_{h}(x-$ $\left.\omega_{h}<1 ; \square-1 \leq \omega_{h}<1.3 ;+-1.3 \leq \omega_{h}<1.8 ; \Delta-\omega_{h} \geq 1.8\right)$. 


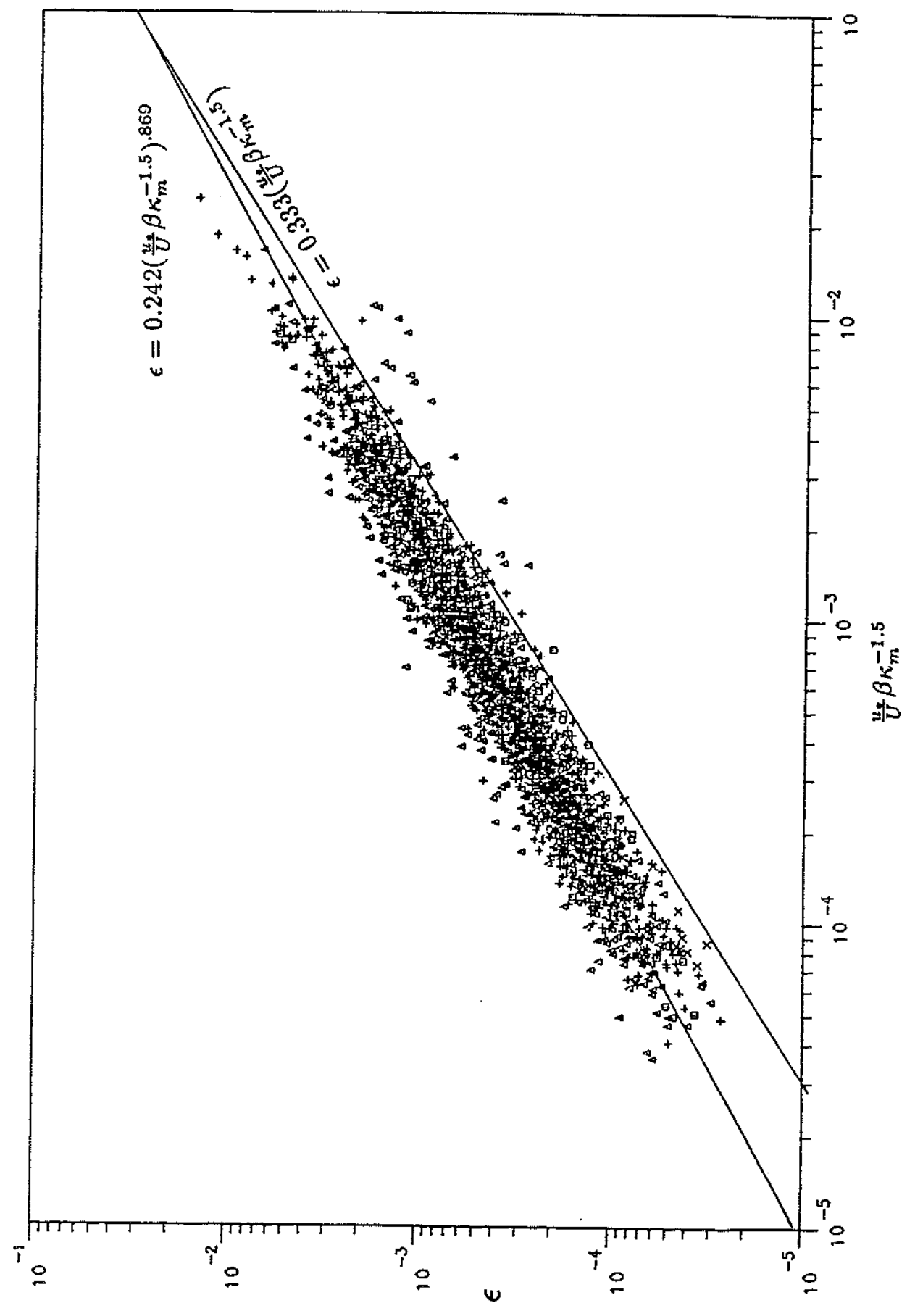

Figure 3-8: Dimensionless energy $\epsilon$ versus scaling parameters $\frac{u_{*}}{U} \beta \kappa_{m}^{-1.5}$ for the entire data set. The two lines represent the regression relation (3.18) and the theoretical relation (3.9). 


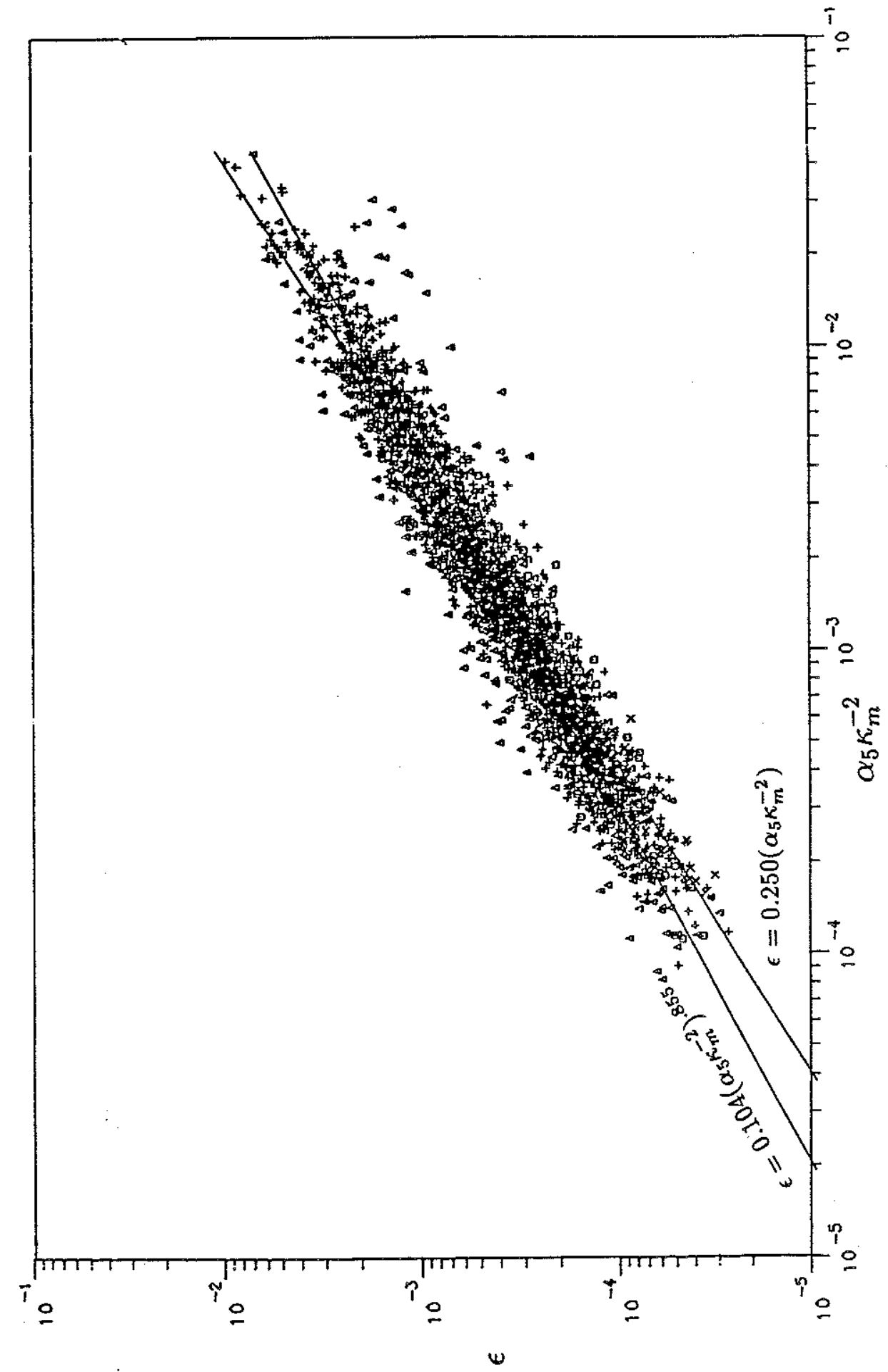

Figure 3-9: Dimensionless energy $\epsilon$ versus scaling parameters $\alpha_{5} \kappa_{m}^{-2}$ for the entire data set. The two lines represent the regression relation (3.18) and the theoretical relation (3.9). 


\begin{tabular}{|c|c|c|c|}
\hline Range & \# of points & Relation with $U$ & Relation with $U_{e}$ \\
\hline$\omega_{h}<1$ & 55 & $\epsilon=0.232\left(\alpha_{5} \kappa_{m}^{-2}\right)^{.992}$ & $\left.\epsilon=0.195 \alpha_{5} \kappa_{m}^{-2}\right)^{.965}$ \\
\hline $1 \leq \omega_{h}<1.3$ & 368 & $\epsilon=0.109\left(\alpha_{5} \kappa_{m}^{-2}\right)^{.865}$ & $\epsilon=0.100\left(\alpha_{5} \kappa_{m}^{-2}\right)^{.847}$ \\
\hline $1.3 \leq \omega_{h}<1.8$ & 2012 & $\epsilon=0.110\left(\alpha_{5} \kappa_{m}^{-2}\right)^{.866}$ & $\epsilon=0.111\left(\alpha_{5} \kappa_{m}^{-2}\right)^{.864}$ \\
\hline$\omega_{h} \geq 1.8$ & 1111 & $\epsilon=0.089\left(\alpha_{5} \kappa_{m}^{-2}\right)^{.825}$ & $\epsilon=0.110\left(\alpha_{5} \kappa_{m}^{-2}\right)^{.851}$ \\
\hline all points & 3546 & $\epsilon=0.104\left(\alpha_{5} \kappa_{m}^{-2}\right)^{.865}$ & $\epsilon=0.110\left(\alpha_{5} \kappa_{m}^{-2}\right)^{.858}$ \\
\hline
\end{tabular}

Table 3.2: Regression relations for $\alpha_{5}$

$$
\epsilon=0.104\left(\alpha_{5} \kappa_{m}^{-2}\right)^{.855}
$$

are compared to the theoretical relations (3.9) and (3.6) in Figs. 3-8 and 3-9 respectively. It seen that in Figures 3-7, 3-8 and 3-9 regression lines compare well with the respective theoretical relations. Using wave directions, based on effective fetch, new values of $\epsilon$ and $\kappa_{m}$ are calculated for wind speeds $U_{e}=U \cos \left(\theta-\theta_{e}\right)$, where $\theta$ and $\theta_{e}$ are the angles of the wind and effective fetch, respectively. A regression analysis based on (3.11) is performed. The results of the regression analysis for $U \equiv|\mathrm{U}|$ and $U_{e} \equiv U \cos \left(\theta-\theta_{e}\right)$ are summarized in Tables $(3.1-3.3)$ for the three formulations. The effect of using the wind component in the wave direction is to decrease the slope and the intercept on $\epsilon$ axis for the range $\omega_{h}<1.3$ and moving the regression line farther away from the theoretical regression line. For $\omega_{h} \geq 1.3$ the regression relations come closer to the theoretical relations when the wind component in the direction of the waves is used. It is interesting to note that the regression relations for the range $\omega_{h}<1.0$ are different from the other ranges in all the three formulations. In the case of the formulation (3.6) the regression line coincides with the theoretical energy relation, but for the formulation (3.9) and (3.10) the regression lines are displaced from the theoretical relation. 


\begin{tabular}{|c|c|c|c|}
\hline Range & \# of points & Relation with $U$ & Relation with $U_{e}$ \\
\hline$\omega_{h}<1$ & 55 & $\epsilon=0.629\left(\frac{u_{*}}{U} \beta \kappa_{m}^{-1.5}\right)^{1.02}$ & $\epsilon=0.488\left(\frac{u_{*}}{U} \beta \kappa_{m}^{-1.5}\right)^{.964}$ \\
\hline $1 \leq \omega_{h}<1.3$ & 368 & $\epsilon=0.247\left(\frac{u_{*}}{U} \beta \kappa_{m}^{-1.5}\right)^{.878}$ & $\epsilon=0.239\left(\frac{u_{*}}{U} \beta \kappa_{m}^{-1.5}\right)^{.862}$ \\
\hline $1.3 \leq \omega_{h}<1.8$ & 2012 & $\epsilon=0.250\left(\frac{u_{*}}{U} \beta \kappa_{m}^{-1.5}\right)^{.876}$ & $\epsilon=0.291\left(\frac{u_{*}}{U} \beta \kappa_{m}^{-1.5}\right)^{.866}$ \\
\hline$\omega_{h} \geq 1.8$ & 1111 & $\epsilon=0.214\left(\frac{u_{*}}{U} \beta \kappa_{m}^{-1.5}\right)^{.845}$ & $\epsilon=0.302\left(\frac{u_{*}}{U} \beta \kappa_{m}^{-1.5}\right)^{.881}$ \\
\hline all points & 3546 & $\epsilon=0.242\left(\frac{u_{*}}{U} \beta \kappa_{m}^{-1.5}\right)^{.869}$ & $\epsilon=0.288\left(\frac{u_{*}}{U} \beta \kappa_{m}^{-1.5}\right)^{.882}$ \\
\hline
\end{tabular}

Table 3.3: Regression relations for $\beta$

\begin{tabular}{|c|c|c|c|}
\hline Range & \# of points & Relation with $U$ & Relation with $U_{e}$ \\
\hline$\omega_{h}<1$ & 55 & $\epsilon=0.624\left(\alpha_{4} \kappa_{m}^{-2}\right)^{1.00}$ & $\epsilon=0.511\left(\alpha_{4} \kappa_{m}^{-2}\right)^{.978}$ \\
\hline $1 \leq \omega_{h}<1.3$ & 368 & $\epsilon=0.246\left(\alpha_{4} \kappa_{m}^{-2}\right)^{.872}$ & $\epsilon=0.223\left(\alpha_{4} \kappa_{m}^{-2}\right)^{.854}$ \\
\hline $1.3 \leq \omega_{h}<1.8$ & 2012 & $\epsilon=0.250\left(\alpha_{4} \kappa_{m}^{-2}\right)^{.875}$ & $\epsilon=0.251\left(\alpha_{4} \kappa_{m}^{-2}\right)^{.872}$ \\
\hline$\omega_{h} \geq 1.3$ & 1111 & $\epsilon=0.212\left(\alpha_{4} \kappa_{m}^{-2}\right)^{.844}$ & $\epsilon=0.261\left(\alpha_{4} \kappa_{m}^{-2}\right)^{.868}$ \\
\hline all points & 3546 & $\epsilon=0.241\left(\alpha_{4} \kappa_{m}^{-2}\right)^{.867}$ & $\epsilon=0.251\left(\alpha_{4} \kappa_{m}^{-2}\right)^{.869}$ \\
\hline
\end{tabular}

Table 3.4: Regression relations for $\alpha_{4}$ 
The CCIW towers recorded data only when the wind speed was above a pre-set threshold. This data set consists of measurements when the wave heights were the largest. A regression analysis of the CCIW tower data (a total of 598 data points) yielded the following relations for $|\mathrm{U}|$

$$
\begin{gathered}
\epsilon=0.352\left(\alpha_{4} \kappa_{m}^{-2}\right)^{.924} \\
\epsilon=0.350\left(\frac{u_{*}}{U} \beta \kappa_{m}^{-1.5}\right)^{.925} \\
\epsilon=0.151\left(\alpha_{5} \kappa_{m}^{-2}\right)^{.921}
\end{gathered}
$$

and for component wind $U \cos \left(\theta-\theta_{e}\right)$

$$
\begin{gathered}
\epsilon=0.318\left(\alpha_{4} \kappa_{m}^{-2}\right)^{.907} \\
\epsilon=0.380\left(\frac{u_{*}}{U} \beta \kappa_{m}^{-1.5}\right)^{.927} \\
\epsilon=0.138\left(\alpha_{5} \kappa_{m}^{-2}\right)^{.904}
\end{gathered}
$$

In Figure 3-10 the scatter plot of $\epsilon$ versus $\alpha_{4} \kappa_{m}^{-2}$ for the Canadian towers is presented with the regression lines (3.20) and (3.23) and the theoretical energy relation (3.10). The slope of the regression line is very close to 1.0 and compares very well with the theoretical energy relation (3.10). From the regression relations (3.21) and (3.22) it is clear that the other two formulations (3.6) and (3.9) have similar characteristics for the Canadian tower data.

The validity of the three formulations $(1.1-1.3)$ can be analyzed by comparing the fit of the data to the three theoretical energy relations $(3.6,3.9$ and 3.10$)$. The summary of the results of the regression analysis is presented for all the three formulations in Table 3.5 with previous available data. From Table 3.5 it is clear that the energy spectrum formulations with a $f^{-4}$ tail (i.e., with equilibrium parameters $\beta$ and $\alpha_{4}$ ) are consistently closer to the theoretical energy relation than the formulation with $f^{-5}$ tail. 


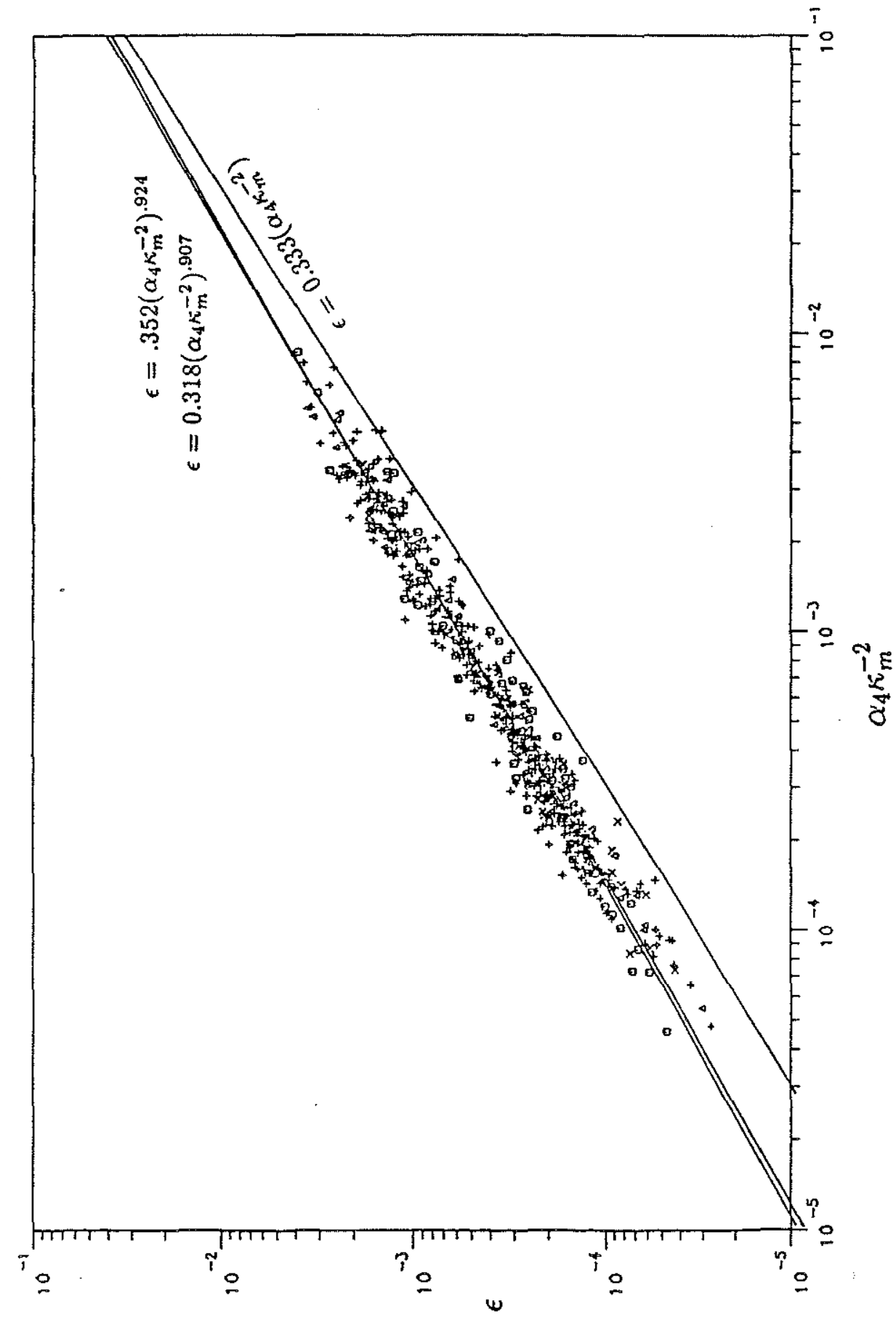

Figure 3-10: Dimensionless energy $\epsilon$ versus scaling parameters $\alpha_{4} \kappa_{m}^{-2}$ for data from CCIW towers only. The three lines represent the regression relations (3.20), (3.23) and the theoretical relation (3.10). 


\begin{tabular}{|c|l|l|l|}
\hline Source & Relation with $\alpha_{4}$ & Relation with $\beta$ & Relation with $\alpha_{5}$ \\
\hline Theory & $\epsilon=0.333\left(\alpha_{4} \kappa_{m}^{-2}\right)$ & $\epsilon=0.333\left(\frac{u_{*}}{U} \beta \kappa_{m}^{-1.5}\right)$ & $\epsilon=0.250\left(\alpha_{5} \kappa_{m}^{-2}\right)$ \\
Bouws et al. & & $\epsilon=0.141\left(\alpha_{5} \kappa_{m}^{-2}\right)^{.880}$ \\
$\begin{array}{c}\text { St. Clair } \\
\text { (entire data) } \\
\begin{array}{c}\text { St.Clair } \\
(\mathrm{C} \text { towers } U)\end{array} \\
\begin{array}{c}\text { St. Clair } \\
\left(\mathrm{C} \text { towers } U_{e}\right)\end{array}\end{array}$ & $\epsilon=0.241\left(\alpha_{4} \kappa_{m}^{-2}\right)^{.867}$ & $\epsilon=0.242\left(\frac{u_{*}}{U} \beta \kappa_{m}^{-1.5}\right)^{.869}$ & $\epsilon=0.104\left(\alpha_{5} \kappa_{m}^{-2}\right)^{.855}$ \\
\hline
\end{tabular}

Table 3.5: Comparison of regression coefficients for the three formulations

\subsection{Spectral and Growth Stage Parameters}

Relations between spectral and growth stage parameters are useful as input in numerical wave prediction models. Studies in deep and finite depth water have provided useful spectral relations that have been extensively used in wave prediction models (Hasselmann et al., 1976; SWIM Group, 1985 ; SWAMP Group, 1985). Hasselmann et al. (1976), using fetch relations obtained from the JONSWAP data and laboratory measurements, found the relation between $\alpha_{5}$ and $\nu$ to be

$$
\alpha_{5}=0.032 \nu^{\frac{2}{3}}
$$

From deep water wave measurements in Lake Ontario, Donelan et al. (1985) observed the spectral parameters $\epsilon$ and $\alpha_{4}$ to be functions of the parameter $U_{e} / c$ described by

$$
\alpha_{4}=6 \times 10^{-3}\left(U_{e} / c\right)^{0.55}
$$

and

$$
\epsilon=2.74 \times 10^{-3}\left(U_{e} / c\right)^{-3.3}
$$

for growing sea states $\left(U_{e} / c>0.82\right)$. The relation (3.27) can be transformed using (1.5) to

$$
\beta=0.068 \nu^{-0.45}
$$


which describes the relation between $\beta$ and $\nu$.

Battjes et al. (1987) reanalyzed the JONSWAP data using 99 of the 121 original spectra used in Hasselmann et al. (1973). Spectral parameters were calculated for the Toba formulation (1.2) and recalculated for the original formulation (1.1). They found the results

$$
\alpha_{5}=0.056 \nu^{1.06}
$$

and

$$
\beta=0.126 \nu^{0.23} \text {. }
$$

The relation found by Battjes et al. (1987) for $\alpha_{5}$ is similar to the one found by Müller (1976) using all the 121 JONSWAP data given by

$$
\alpha_{5}=0.05 \nu^{1.17}
$$

but is very different from the relation (3.26) that was derived based on a set of laboratory and field measurements (Hasselmann et al., 1973).

Bouws et al. (1987) analyzed the spectral parameters $\epsilon$ and $\alpha_{5}$ as functions of the three growth stage parameters $\nu, U / c$ and $\kappa_{m}$. The data used were from three different wave growth studies in finite depth (see Bouws et al., 1985). The results showed that there was a significant variation with depth for $\epsilon, \alpha_{5}-U / c$ and $\epsilon, \alpha_{5}-\nu$ relations, but the $\alpha_{5}-\kappa_{m}$ relation had no explicit depth dependence and was given by

$$
\alpha_{5}=0.0078 \kappa_{m}^{0.49}
$$

A power law relationship between the spectral parameter (say $a$ ) and the growth stage parameter (say $\nu$ ) of the form

$$
a=r \nu^{s}
$$

is assumed and using a linear regression analysis with logarithmic scales the coefficients are calculated from the data set. Following Bouws et al. (1987) regression relations between 


\begin{tabular}{|c|c|c|c|c|c|c|c|c|}
\hline & \multicolumn{4}{|c|}{$\epsilon$ vs $\nu$} & \multicolumn{4}{c|}{$\alpha_{5}$ vs $\nu$} \\
\hline & \multicolumn{2}{|c|}{ Bouws $e t$ al. } & \multicolumn{2}{|c|}{ Lake St.Clair } & \multicolumn{2}{|c|}{ Bouws et al. } & \multicolumn{2}{c|}{ Lake St.Clair } \\
\hline Range & $a \times 10^{6}$ & $r$ & $a \times 10^{6}$ & $r$ & $a$ & $r$ & $a$ & $r$ \\
\hline$\omega_{h}<0.7$ & 25.0 & -2.05 & & & 0.078 & 1.05 & & \\
\hline $0.7 \leq \omega_{h}<1$ & 9.60 & -2.67 & 1.84 & -3.77 & 0.045 & 0.94 & 0.021 & 0.34 \\
\hline $1 \leq \omega_{h}<1.3$ & 16.6 & -2.48 & 3.79 & -3.41 & 0.036 & 0.83 & 0.015 & 0.13 \\
\hline$\omega_{h} \geq 1.3$ & 9.56 & -2.93 & 4.79 & -3.33 & 0.030 & 0.66 & 0.014 & 0.15 \\
\hline all & 34.3 & -2.05 & 4.55 & -3.36 & 0.032 & 0.71 & 0.015 & 0.15 \\
\hline
\end{tabular}

Table 3.6: Regression relations for $\epsilon$ vs $\nu$

$\epsilon$ and $\alpha_{5}$ and the growth stage parameters $\nu, U / c$ and $\kappa_{m}$ are calculated. In Tables 3.6 - 3.8, the regression relations obtained are compared with the results from Bouws et al. (1987). The results are tabulated on a classification based on the dimensionless depth $\omega_{h}$, with ranges that are different from the ranges used in previous tabulations. The relations for $\epsilon$ in terms of $\nu$ and $U / c$ have larger negative slopes than the results in Bouws et al. (1987). There is also less variation in the relations as a function of depth for Lake St. Clair data. The variation in $\alpha_{5}$ as a function of the three growth stage parameters is negligible whereas Bouws et al. (1987) observed increasing values of $\alpha_{5}$ for increasing $\nu, U / c$ and $\kappa_{m}$. The variation of energy in terms of $\kappa_{m}$ compares well with values observed by Bouws et al. (1987) and show very little variation with depth.

In Figure 3-11 the values of $\alpha_{5}$ is plotted against $\nu$ and the regression relation

$$
\alpha_{5}=0.014 \nu^{0.15}
$$

is compared to JONSWAP result (3.19) and to (3.24). The results from Lake St.Clair show 


\begin{tabular}{|c|c|c|c|c|c|c|c|c|}
\hline & \multicolumn{4}{|c|}{$\epsilon$ vs $U / c$} & \multicolumn{4}{c|}{$\alpha_{5}$ vs $U / c$} \\
\hline & \multicolumn{2}{|c|}{ Bouws et al. } & Lake St.Clair & \multicolumn{3}{c|}{ Bouws et al } & \multicolumn{2}{c|}{ Lake St.Clair } \\
\hline Range & $\mathrm{a} \times 10^{3}$ & $\mathrm{r}$ & $\mathrm{a} \times 10^{3}$ & $\mathrm{r}$ & $\mathrm{a} \times 10^{3}$ & $\mathrm{I}$ & $\mathrm{a} \times 10^{3}$ & $\mathrm{I}$ \\
\hline$\omega_{h}<0.7$ & 4.43 & -2.19 & & & 4.90 & 1.46 & & \\
\hline $0.7 \leq \omega_{h}<1$ & 2.87 & -2.64 & 3.53 & -3.49 & 6.17 & 0.93 & 10.5 & 0.33 \\
\hline $1 \leq \omega_{h}<1.3$ & 2.10 & -2.50 & 2.64 & -3.32 & 7.14 & 0.83 & 11.4 & 0.12 \\
\hline$\omega_{h} \geq 1.3$ & 2.21 & -2.99 & 2.32 & -3.35 & 8.73 & 0.65 & 10.9 & 0.15 \\
\hline all & 2.40 & -2.12 & 2.33 & -3.32 & 7.14 & 0.83 & 10.9 & 0.16 \\
\hline
\end{tabular}

Table 3.7: Regression relations for $\epsilon$ vs $U / c$

that $\alpha_{5}$ is nearly constant indicating no systematic variation with growth stage parameter $\nu$ whereas the results of Battjes et al. (1987) and Hasselmann et al. (1976) show an increase in $\alpha_{5}$ with increasing $\nu$. The scatter plot of $\beta$ versus $\nu$ is given in Figure 3-12 along with the regression relation

$$
\beta=0.022 \nu^{-1.04}
$$

and compared to the relations (3.29) and (3.31). Battjes et al. (1987) result indicates a nearly constant $\beta$ while the Lake St.Clair results indicate a larger decrease with growth stage parameter $\nu$ than that observed by Donelan et al. (1985). The relation between energy and the parameter $U_{e} / c$

$$
\epsilon=0.0025\left(U_{e} / c\right)^{-3.36}
$$

is plotted along with (3.28) in Figure 3-13. The variation of energy is in good agreement with deep water observations of Donelan et al. (1985). A plot of the equilibrium parameter 


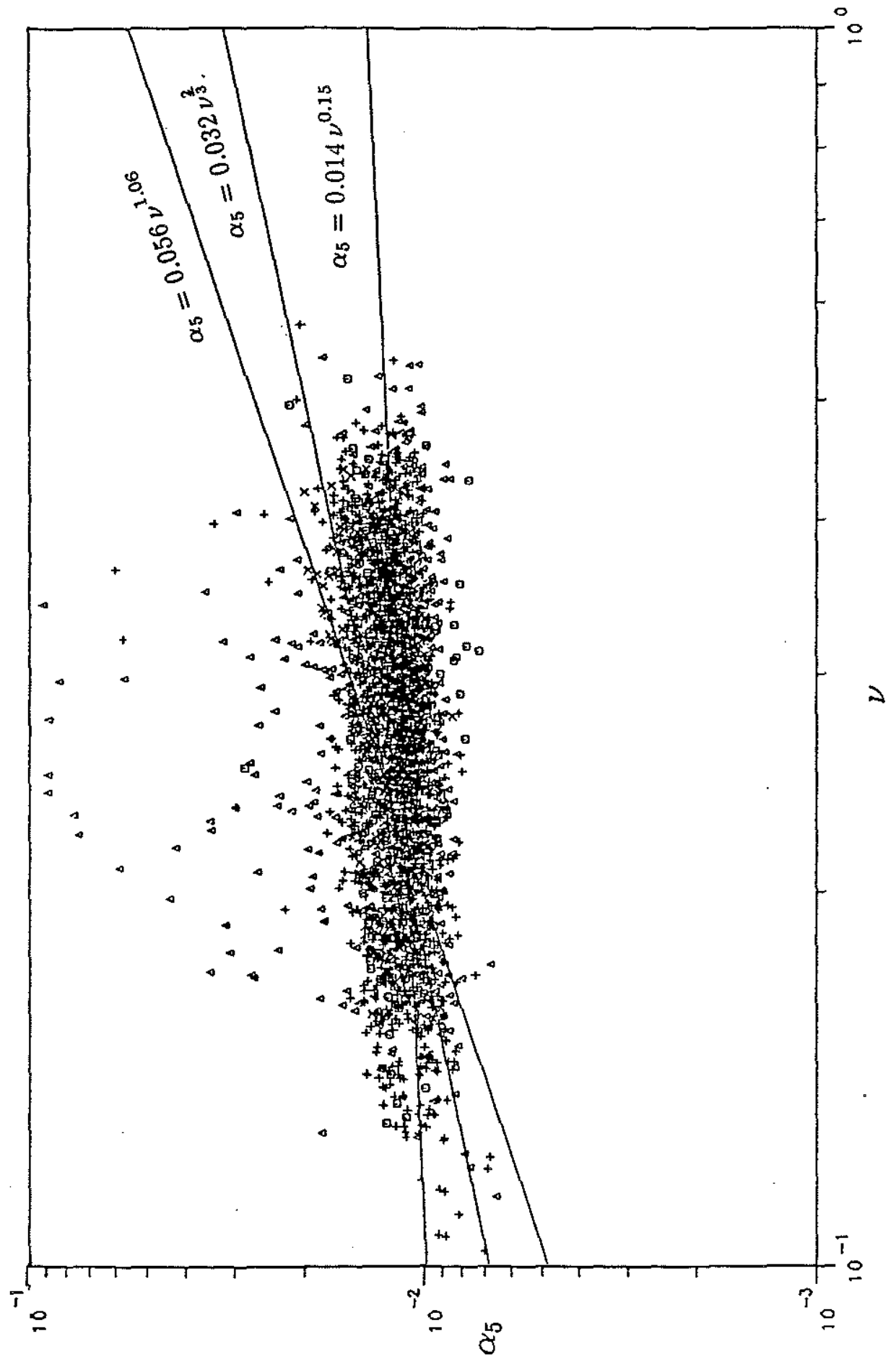

Figure 3-11: Equilibrium parameter $\alpha_{5}$ versus nondimensional peak frequency $\nu$ for the entire data set. The three lines represent the regression relation (3.35), the JONSWAP relation (3.26) and Toba's relation, $(3.30)$. 


\begin{tabular}{|c|c|c|c|c|c|c|c|c|}
\hline & \multicolumn{4}{|c|}{$\epsilon$ vs $\kappa_{m}$} & \multicolumn{4}{c|}{$\alpha_{5}$ vs $\kappa_{m}$} \\
\hline & \multicolumn{2}{|c|}{ Bouws et al. } & \multicolumn{2}{c|}{ Lake St.Clair } & \multicolumn{2}{c|}{ Bouws et al. } & \multicolumn{2}{c|}{ Lake St.Clair } \\
\hline Range & $\mathrm{a} \times 10^{3}$ & $\mathrm{r}$ & $\mathrm{a} \times 10^{3}$ & $\mathrm{r}$ & $\mathrm{a} \times 10^{3}$ & $\mathrm{r}$ & $\mathrm{a} \times 10^{3}$ & $\mathrm{r}$ \\
\hline$\omega_{h}<0.7$ & 2.18 & -1.19 & & & 7.99 & 0.68 & & \\
\hline $0.7 \leq \omega_{h}<1$ & 1.92 & -1.36 & 2.68 & -1.83 & 7.15 & 0.48 & 10.8 & 0.17 \\
\hline $1 \leq \omega_{h}<1.3$ & 1.80 & -1.26 & 2.31 & -1.68 & 7.52 & 0.42 & 11.4 & 0.06 \\
\hline$\omega_{h} \geq 1.3$ & 2.11 & -1.48 & 2.26 & -1.67 & 8.77 & 0.33 & 10.9 & 0.07 \\
\hline all & 1.98 & -1.34 & 2.27 & -1.67 & 7.76 & 0.49 & 10.9 & 0.08 \\
\hline
\end{tabular}

Table 3.8: Regression relations for $\epsilon$ vs $\kappa_{m}$

$\alpha_{4}$ versus $U_{e} / c$ is given in Fig. 3-14. The regression relation

$$
\alpha_{4}=0.0046\left(U_{e} / c\right)^{0.16}
$$

predicts a slower increase of the parameter $\alpha_{4}$ with increasing $U_{e} / c$ values compared to (3.27) given by Donelan et al. (1985) for deep water. Bouws et al. (1987) formulation (3.33) is plotted along with $\alpha_{5}-\kappa_{m}$ regression relation

$$
\alpha_{5}=0.011 \kappa_{m}^{0.08}
$$

in the scatter plot of $\alpha_{5}$ versus $\kappa_{m}$ in Fig. 3-15. Bouws et al. (1987) results indicate a steep increase in the parameter $\alpha_{5}$ with $\kappa_{m}$ but the results here indicate a nearly constant $\alpha_{5}$.

\subsection{Fetch Relations}

The development of a wave spectrum with fetch is studied here by analysing the relations between spectral parameters and fetch. In the JONSWAP experiment Hasselmann et 


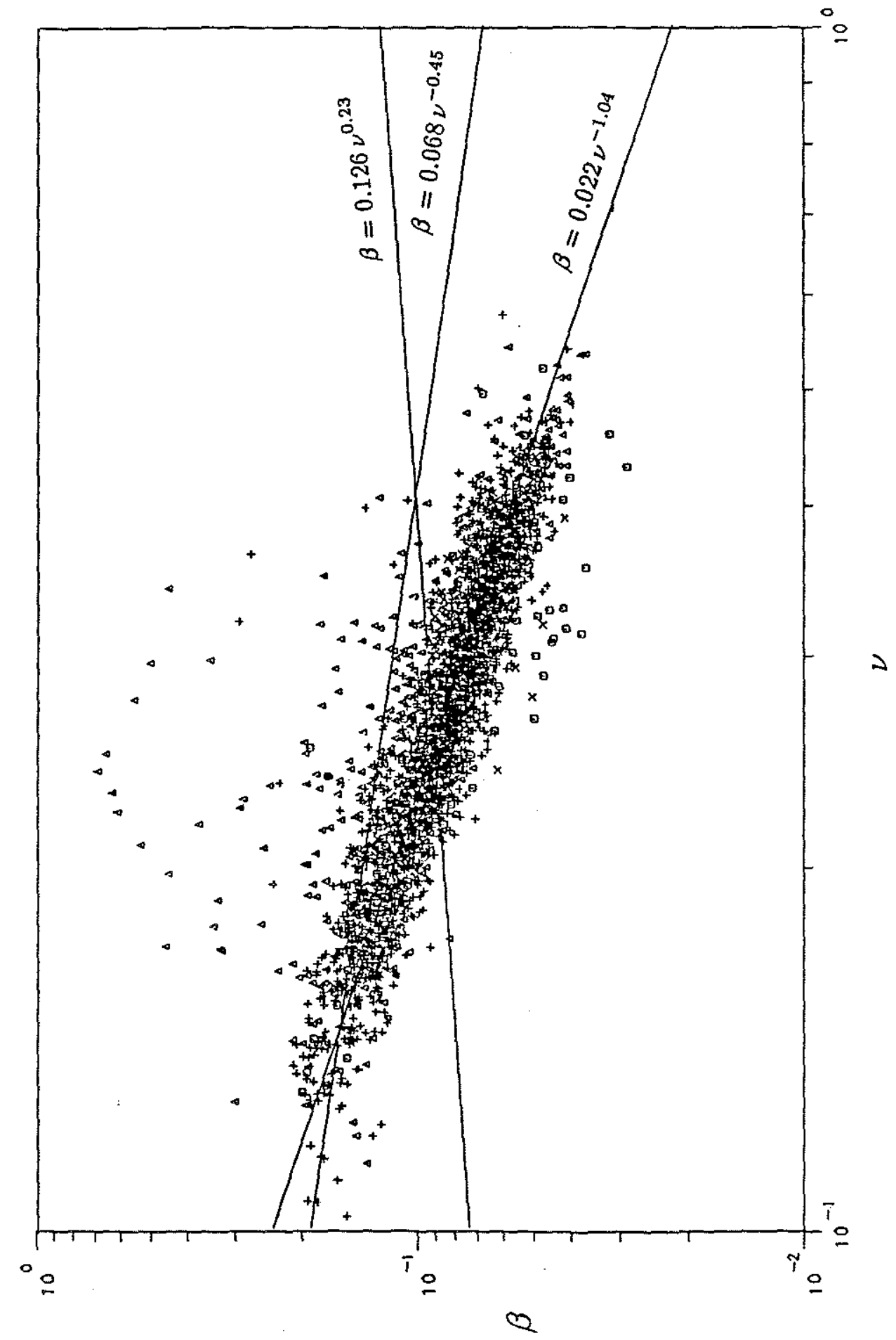

Figure 3-12: Equilibrium parameter $\beta$ versus nondimensional peak frequency $\nu$ for the entire data set. The three lines represent the regression relation (3.36), Donelan's relation (3.26) and Toba's relation (3.31). 


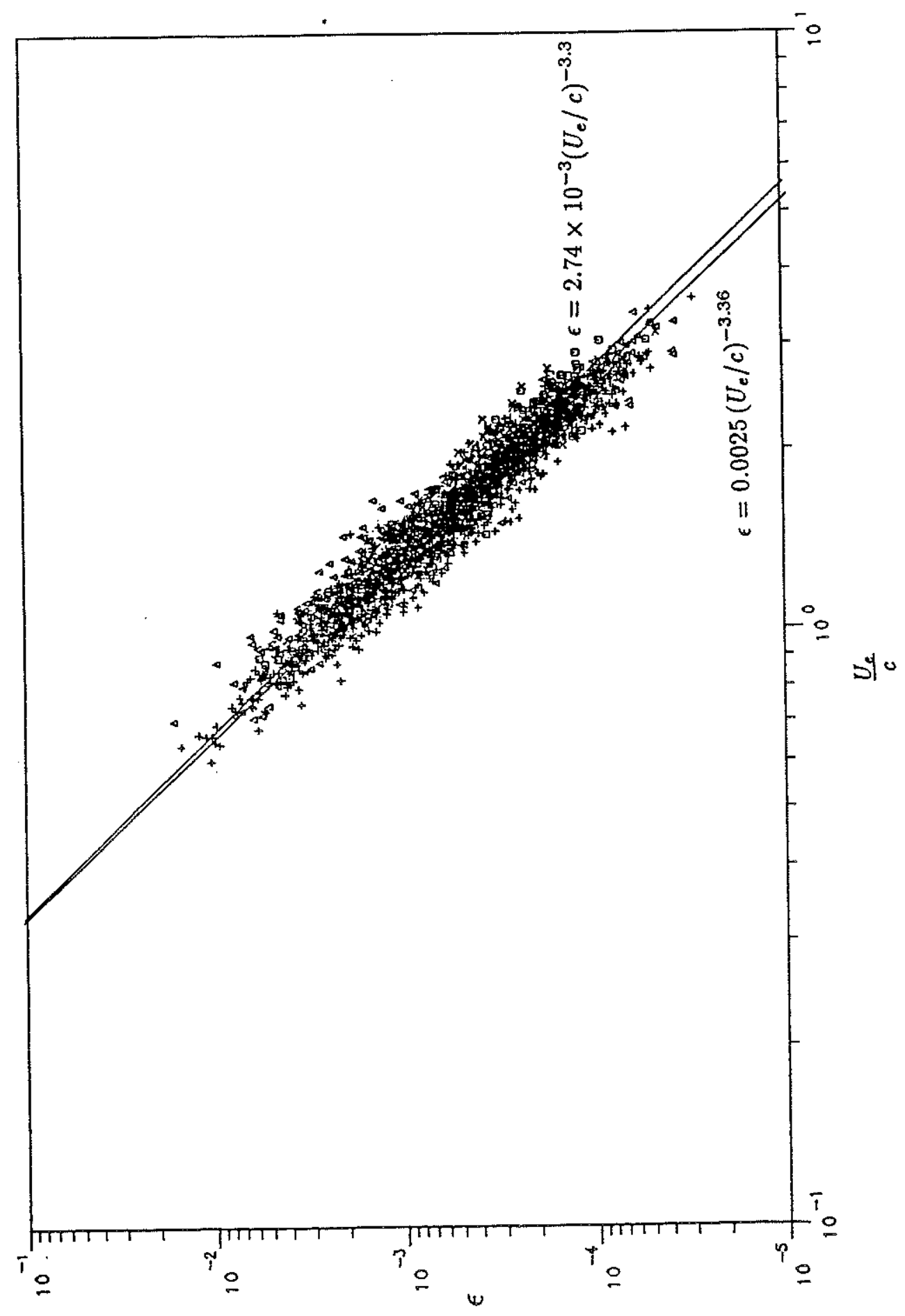

Figure 3-13: Dimensionless energy $\epsilon$ versus relative wind input $U_{c} / c$. The two lines represent the regression relation (3.37) and Donelan's observation (3.28). 


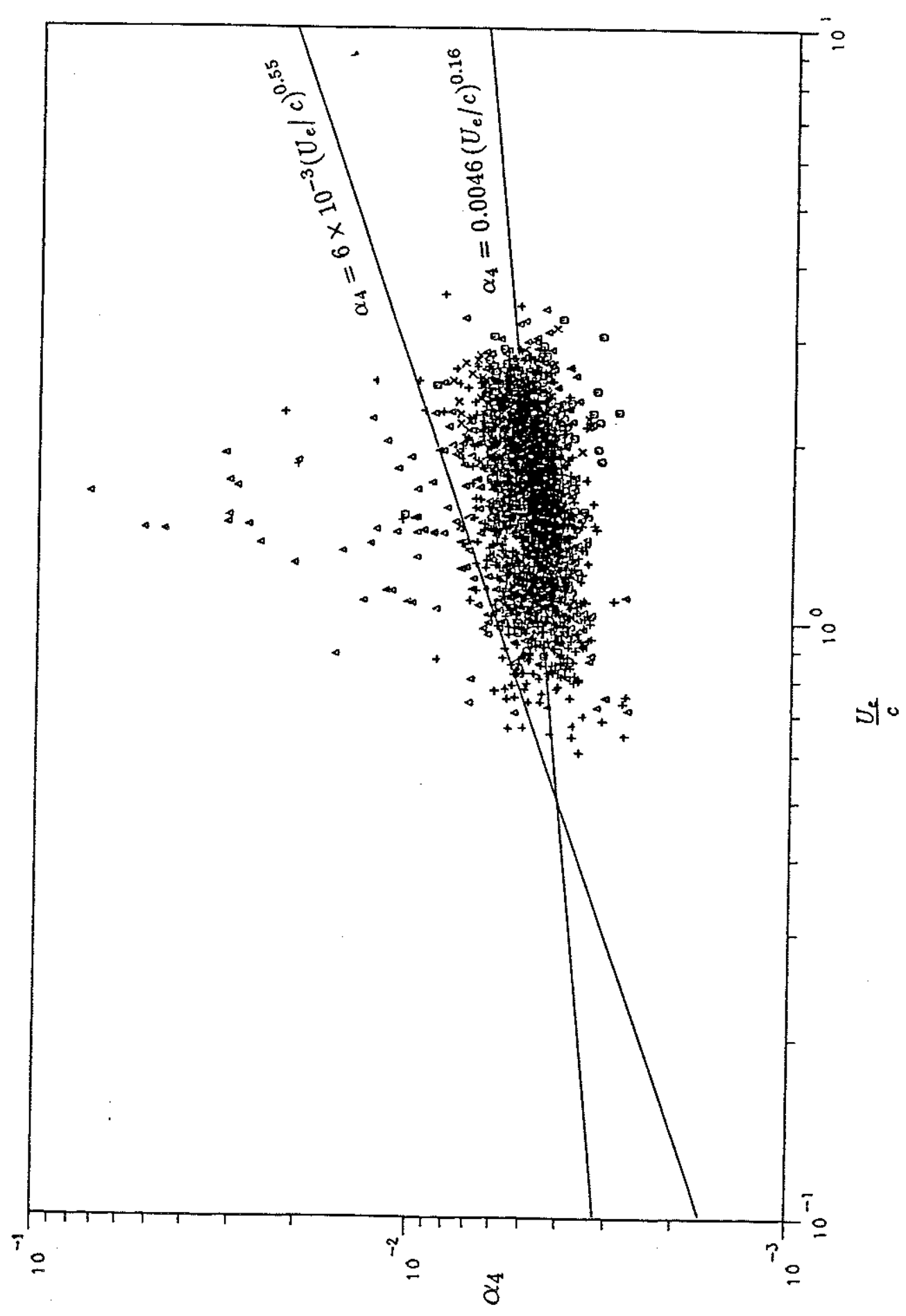

Figure 3-14: Equilibrium parameter $\alpha_{4}$ versus relative wind input $U_{e} / c$. for the entire data set. The two lines represent the regression relation (3.38) and Donelan's observation (3.27). 


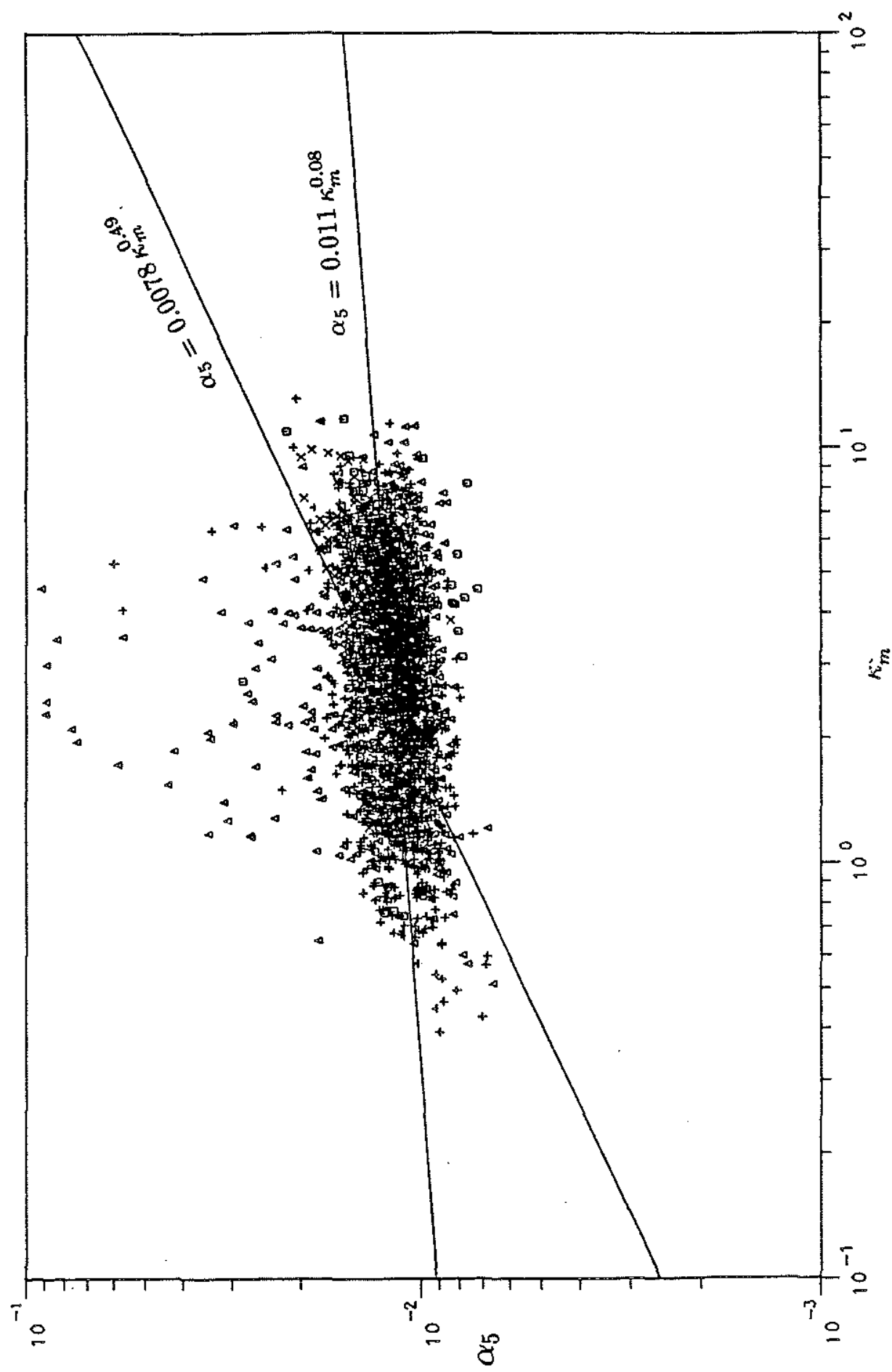

Figure 3-15: Equilibrium parameter $\alpha_{5}$ versus dimensionless wave number $\kappa$ for the entire data set. The two lines represent the regression ${ }_{m}$ relation (3.39) and Bouws's observation (3.33). 
al. (1973) found that in deep water the peak spectral frequency $f_{m}$ and the equilibrium parameter $\alpha_{5}$ depend explicitly on fetch and wind speed. The following relations for the dimensionless frequency $\nu$, total energy $\epsilon$ and $\alpha_{5}$ in terms of $\xi$, the dimensionless fetch

$$
\xi \equiv \frac{g X}{U^{2}}
$$

where $X$ is the fetch, were obtained from the JONSWAP data.

$$
\begin{gathered}
\nu=3.5 \xi^{-0.33} \\
\alpha_{5}=0.076 \xi^{-0.22} \\
\epsilon=1.6 \times 10^{-7} \xi .
\end{gathered}
$$

Kahma (1981) studied the growth of waves with fetch using measurements from a line of wave buoys off the coast of Finland and observed that the nondimensional wave frequency obeyed the same functional form, but the nondimensional wave energy was nearly double of the energy observed in the JONSWAP experiments. The relations Kahma (1981) observed were

$$
\nu=3.18 \xi^{-0.33}
$$

and

$$
\epsilon=3.6 \times 10^{-7} \xi
$$

Donelan et al. (1985) proposed the following fetch relations from measurements in Lake Ontario.

$$
\begin{gathered}
\epsilon=8.41 \times 10^{-7} \xi^{0.76} \\
\nu=1.85 \xi^{-0.23} \\
\alpha_{4}=0.023 \xi^{-0.13}
\end{gathered}
$$


These relations are valid for the range $0.83<U_{e} / c<5$ and were obtained by substituting the relation between fetch and the parameter $U_{e} / c$

$$
U_{e} / c=11.6 \xi^{-0.23}
$$

in (3.27) and (3.28), the equations that related $\alpha_{4}$ and energy to $U_{e} / c$. The limit $U_{e} / c=$ 0.83 corresponds to the wave number limit $\kappa_{m}=0.667$ and frequency limit $\nu=0.13$ that define a fully- developed sea state.

A power-law relationship between the spectral parameter (say $a$ ) and the nondimensional fetch $\xi$ of the form

$$
a=r \xi^{s}
$$

is assumed and using a linear regression analysis for logarithmic scales the coefficients are calculated. Using the effective fetch for each wave station the coefficients of the relation for the spectral parameters $\epsilon, \nu, \alpha_{5}, \alpha_{4}, \kappa_{m}$ and $\beta$ are calculated. The wind component in the wave direction $U_{e}=U \cos \left(\theta-\theta_{e}\right)$ is used in these calculations. The regression relations are divided into classes based on the nondimensional depth and the regression coefficients $r$ and $s$ in (3.49) are given in Table (3.9) for $\epsilon$ and $\nu$ and in Table (3.10) for $\alpha_{4}, \beta$ and $\alpha_{5}$. From the tables it is clear that for $\omega_{h}<1.0$ the relations obtained are different from the relations for the other ranges indicating a dependence on depth.

The relations for energy and peak frequency obtained for this data set are

$$
\begin{gathered}
\nu=2.47 \xi^{-0.28} \\
\epsilon=2.29 \times 10^{-7} \xi^{0.94} .
\end{gathered}
$$

In Figures 3-16 the spectral parameter $\epsilon$ is plotted versus fetch $\xi$ on a $\log -\log$ scale. The relation obtained by the regression analysis is presented along with previous results (3.43), (3.45) and (3.46). The energy fetch relation obtained in this analysis (see Fig 3-16) is very close to one observed in JONSWAP experiment (3.43). The growth of the nondimensional 


\begin{tabular}{|c|c|c|c|c|c|}
\hline Range & pts & \multicolumn{2}{|c|}{$\epsilon$} & \multicolumn{2}{c|}{$\nu$} \\
\hline & & $\mathrm{a} 10^{7}$ & $\mathrm{r}$ & $\mathrm{a}$ & $\mathrm{r}$ \\
\hline$\omega_{h}<1$ & 55 & 1.30 & 0.72 & 1.13 & -0.19 \\
\hline $1.0 \leq \omega_{h}<1.3$ & 368 & 1.95 & 0.97 & 2.63 & -0.29 \\
\hline $1.3 \leq \omega_{h}<1.8$ & 2012 & 2.19 & 0.95 & 2.68 & -0.29 \\
\hline$\omega_{h} \geq 1.8$ & 1111 & 2.38 & 0.94 & 2.24 & -0.26 \\
\hline
\end{tabular}

Table 3.9: Regression relations for $\epsilon$ and $\nu$ versus fetch $\xi$

\begin{tabular}{|c|c|c|c|c|c|c|c|}
\hline Range & $\mathrm{pts}$ & \multicolumn{2}{|c|}{$\alpha_{4}$} & \multicolumn{2}{c|}{$\alpha_{5}$} & \multicolumn{2}{c|}{$\beta$} \\
\hline & & $\mathrm{a}$ & $\mathrm{r}$ & $\mathrm{a}$ & $\mathrm{r}$ & $\mathrm{a}$ & $\mathrm{r}$ \\
\hline$\omega_{h}<1.0$ & 55 & 0.012 & -0.1 & 0.007 & 0.32 & 0.027 & -0.08 \\
\hline $1.0 \leq \omega_{h}<1.3$ & 368 & 0.006 & -0.03 & 0.004 & 0.37 & 0.015 & -0.02 \\
\hline $1.3 \leq \omega_{h}<1.8$ & 2012 & 0.008 & -0.07 & 0.009 & 0.26 & 0.019 & -0.06 \\
\hline$\omega_{h} \geq 1.8$ & 1111 & 0.005 & -0.02 & 0.009 & 0.27 & 0.013 & -0.008 \\
\hline
\end{tabular}

Table 3.10: Regression relations for $\alpha_{4}, \beta$ and $\alpha_{5}$ versus fetch $\xi$ 


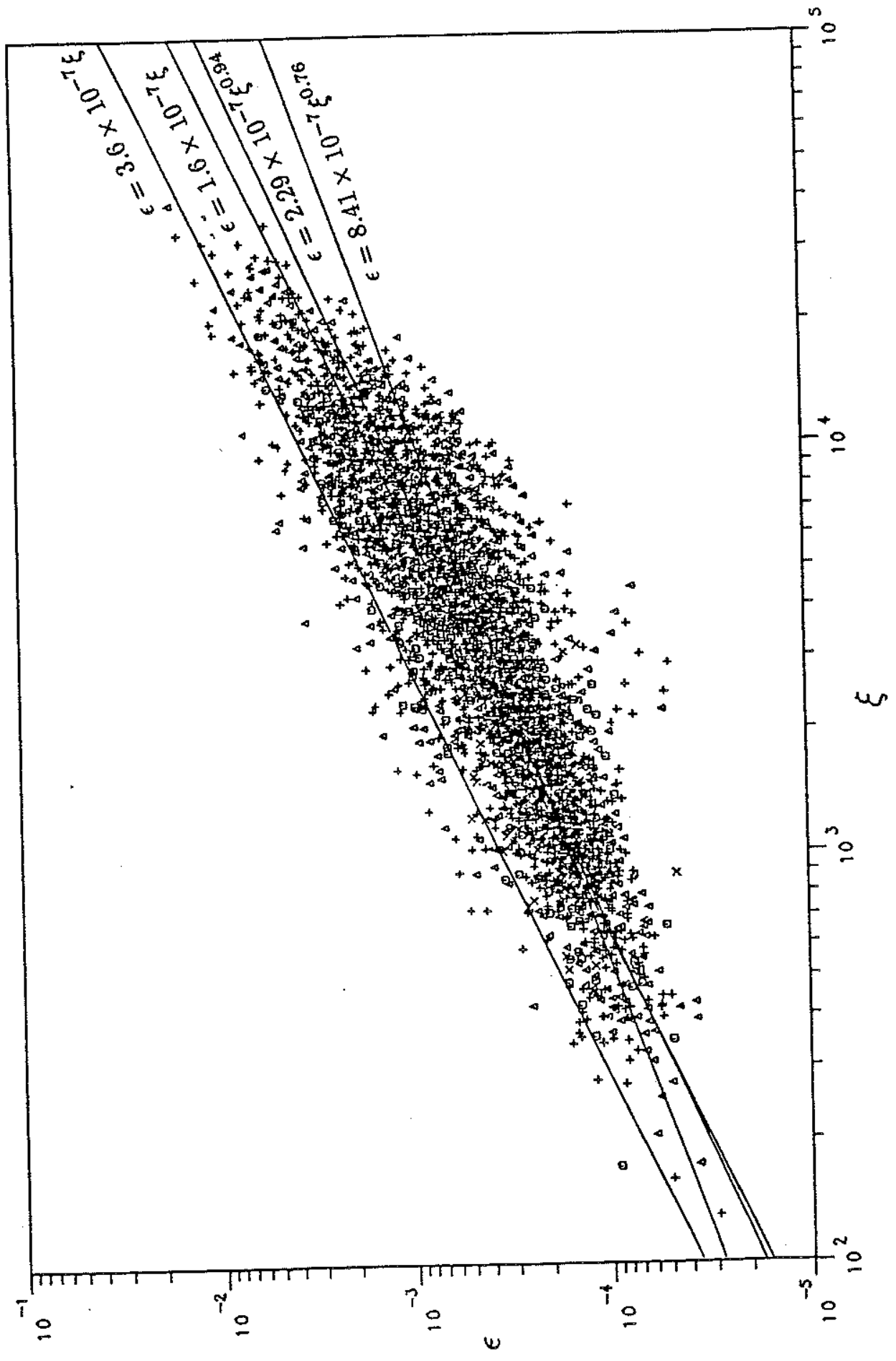

Figure 3-16: Dimensionless energy $\epsilon$ versus dimensionless fetch $\xi$. The four lines represent the regression relation (3.51), Donelan's observation (3.46), Kahma's relation (3.45) and the JONSWAP relation (3.43). 
frequency $\nu$ as function of nondimensional fetch is given in Fig. 3-17. The regression relation (3.50) is compared to (3.41), (3.44) and (3.47). For small fetch ranges all four relations compare well, but diverge from one another at large fetch. The growth of energy in Lake St. Clair is larger than that observed by Donelan et al. (1985) but is lower than that observed by Hasselmann et al. (1973) and Kahma (1981). The peak frequency is lower than the values observed by Donelan et al. (1985) but larger than that given by Kahma (1981) and Hasselmann et al. (1973).

The plots of the equilibrium parameters $\alpha_{5}, \alpha_{4}$ and $\beta$ versus the nondimensional fetch $\xi$ are presented in Figures 3-18, 3-19 and 3-20, respectively. The regression analysis yielded the relations

$$
\begin{gathered}
\alpha_{5}=0.017 \xi^{-0.04} \\
\alpha_{4}=0.007 \xi^{-0.05} \\
\beta=0.009 \xi^{0.28} .
\end{gathered}
$$

In Figure 3-18, (3.52) is compared with the JONSWAP result (3.42). For increasing fetch a nearly constant value of $\alpha_{5}$ for the Lake St. Clair data is observed unlike a slight decrease observed in the JONSWAP data. The regression relation (3.53) for the growth of $\alpha_{4}$ shows a slight decrease with fetch (Figure 3-19) but is displaced from the result (3.48) observed by Donelan et al. (1985) for deep water. The regression line (3.54) describing the growth of the parameter $\beta$ is given along with the scatter plot of $\beta$ and $\xi$ for the data set in Fig.3-20. There is an appreciable increase in the values of $\beta$ as fetch increases. 


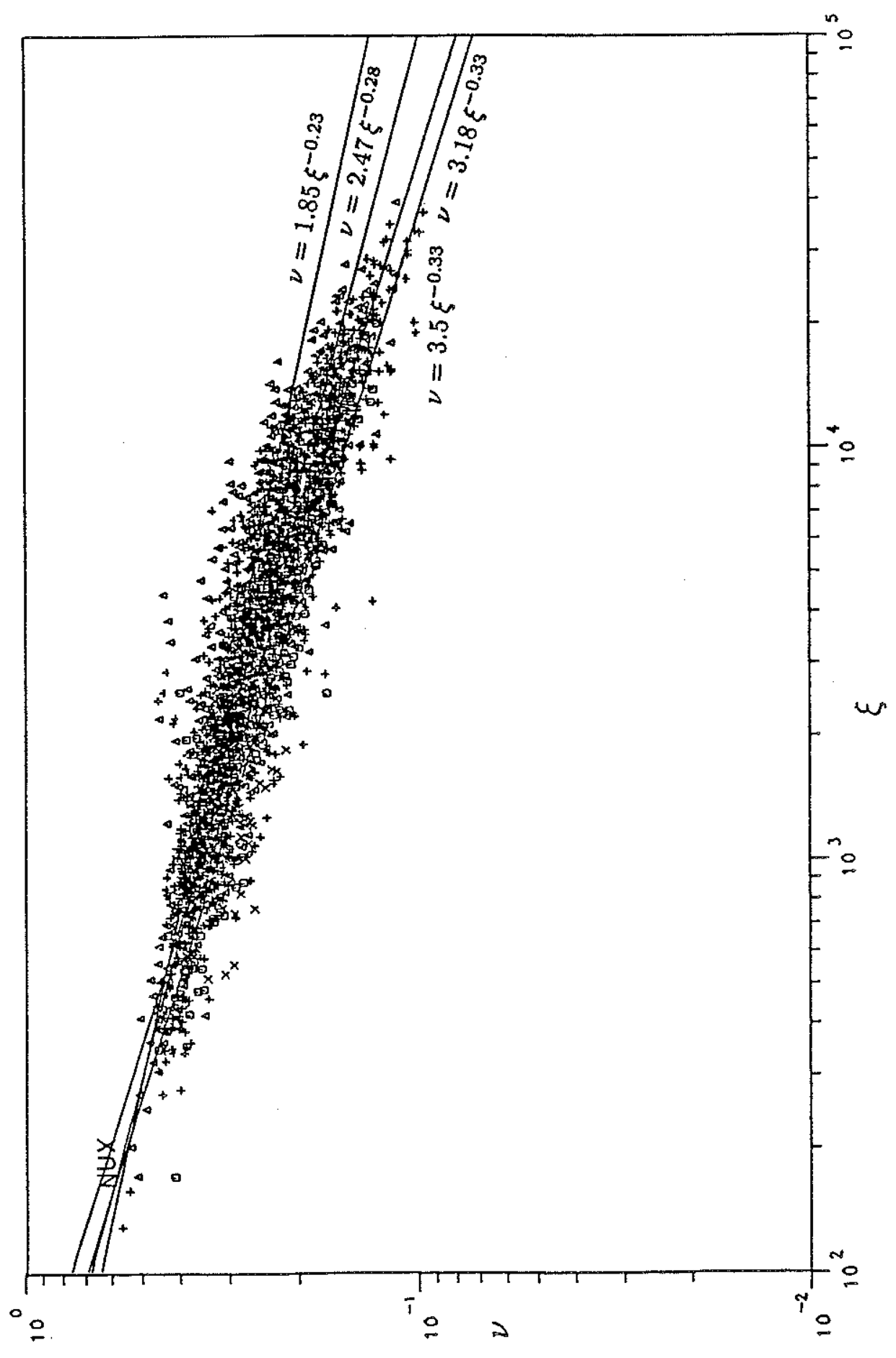

Figure 3-17: Dimensionless peak frequency $\nu$ versus dimensionless fetch $\xi$. The four lines represent the regression relation (3.50); Donelan's observation (3.47), Kahma's relation (3.44) and the JONSWAP relation (3.41). 


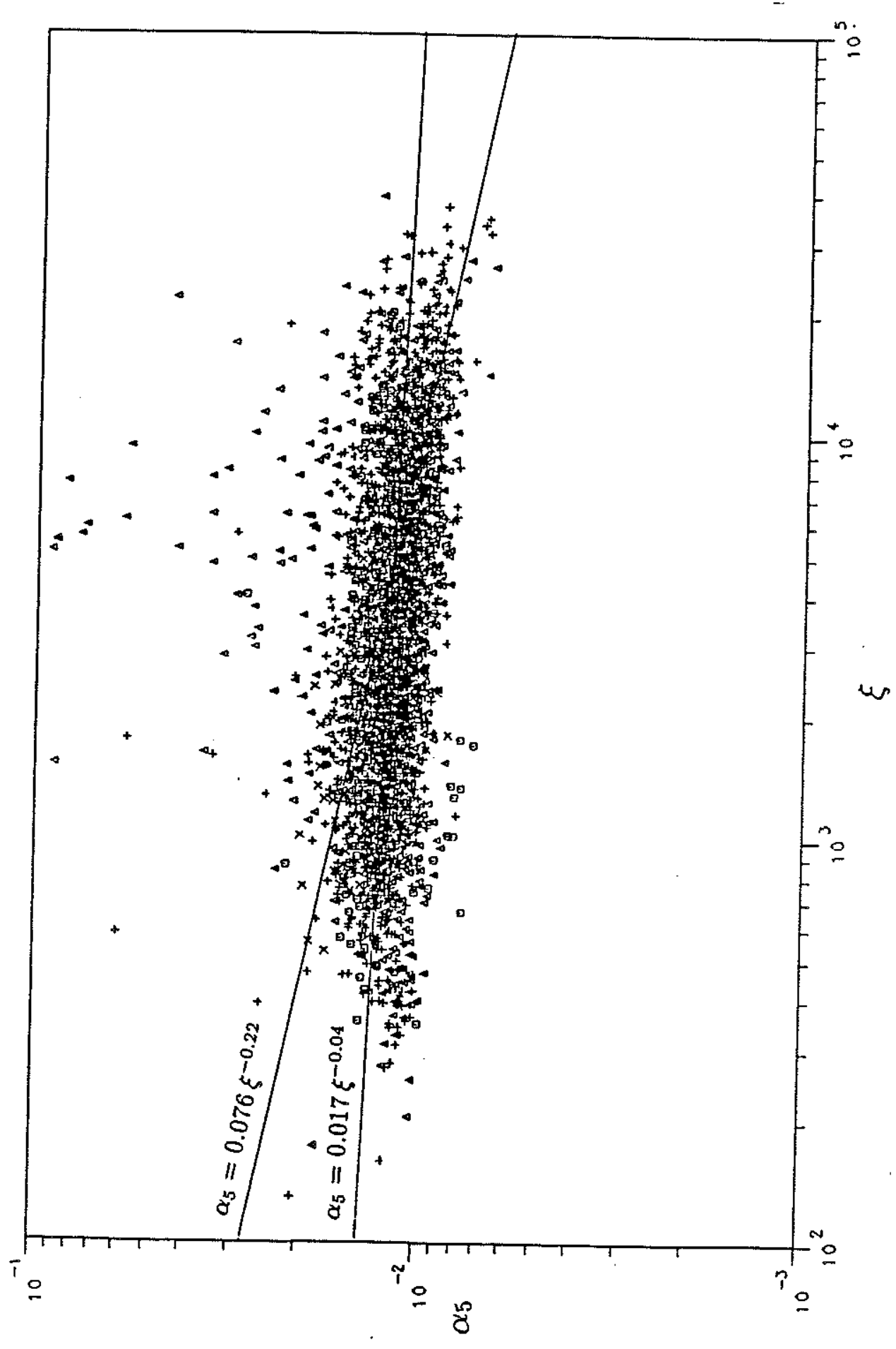

Figure 3-18: Equilibrium parameter $\alpha_{5}$ versus dimensionless fetch $\xi$. The two lines represent the regression relation (3.52) and the JONSWAP relation (3.42). 


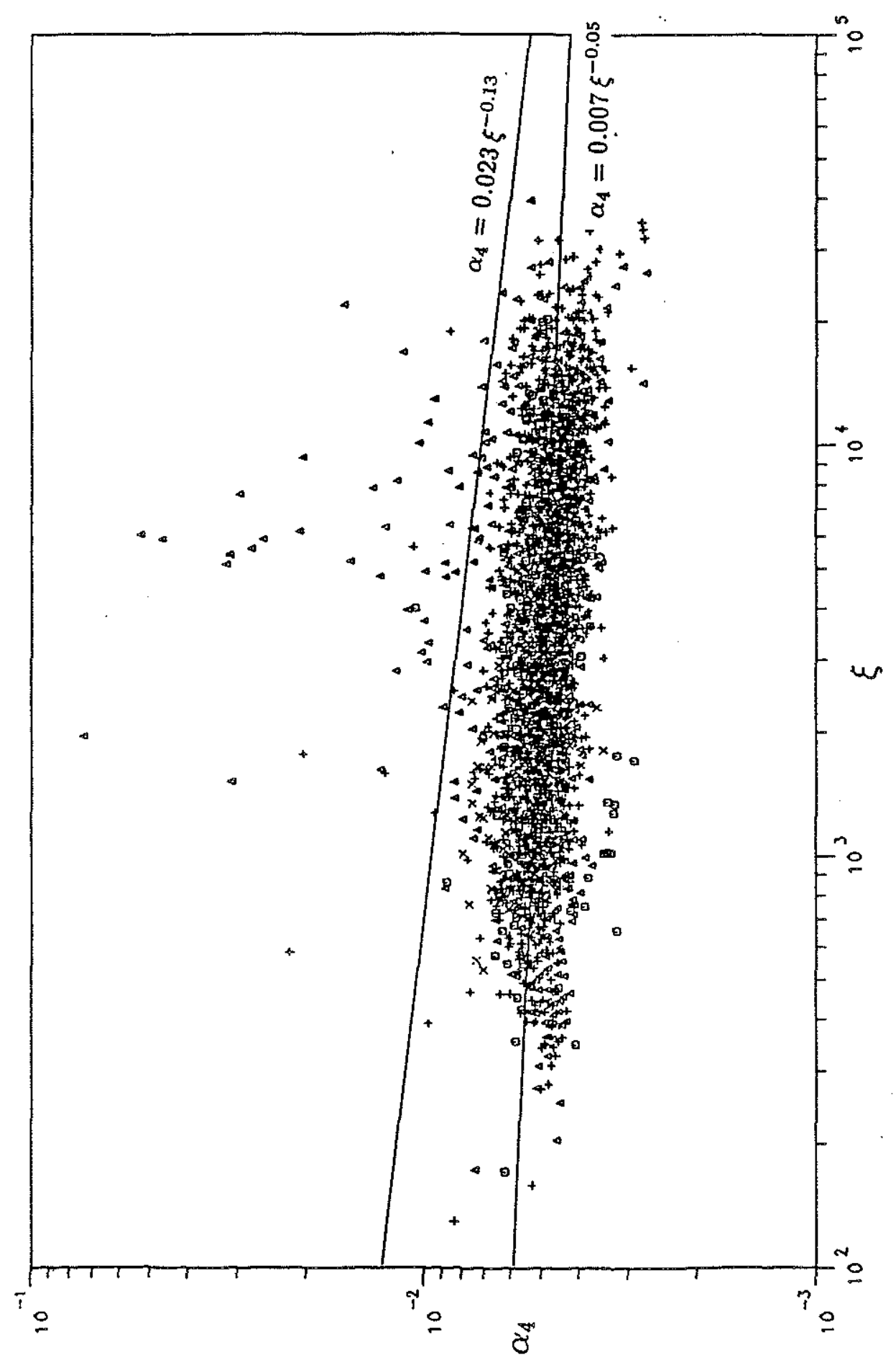

Figure 3-19: Equilibrium parameter $\alpha_{4}$ versus dimensionless fetch $\xi$. The two lines represent the regression relation (3.53) and Donelan's relation (3.48). 


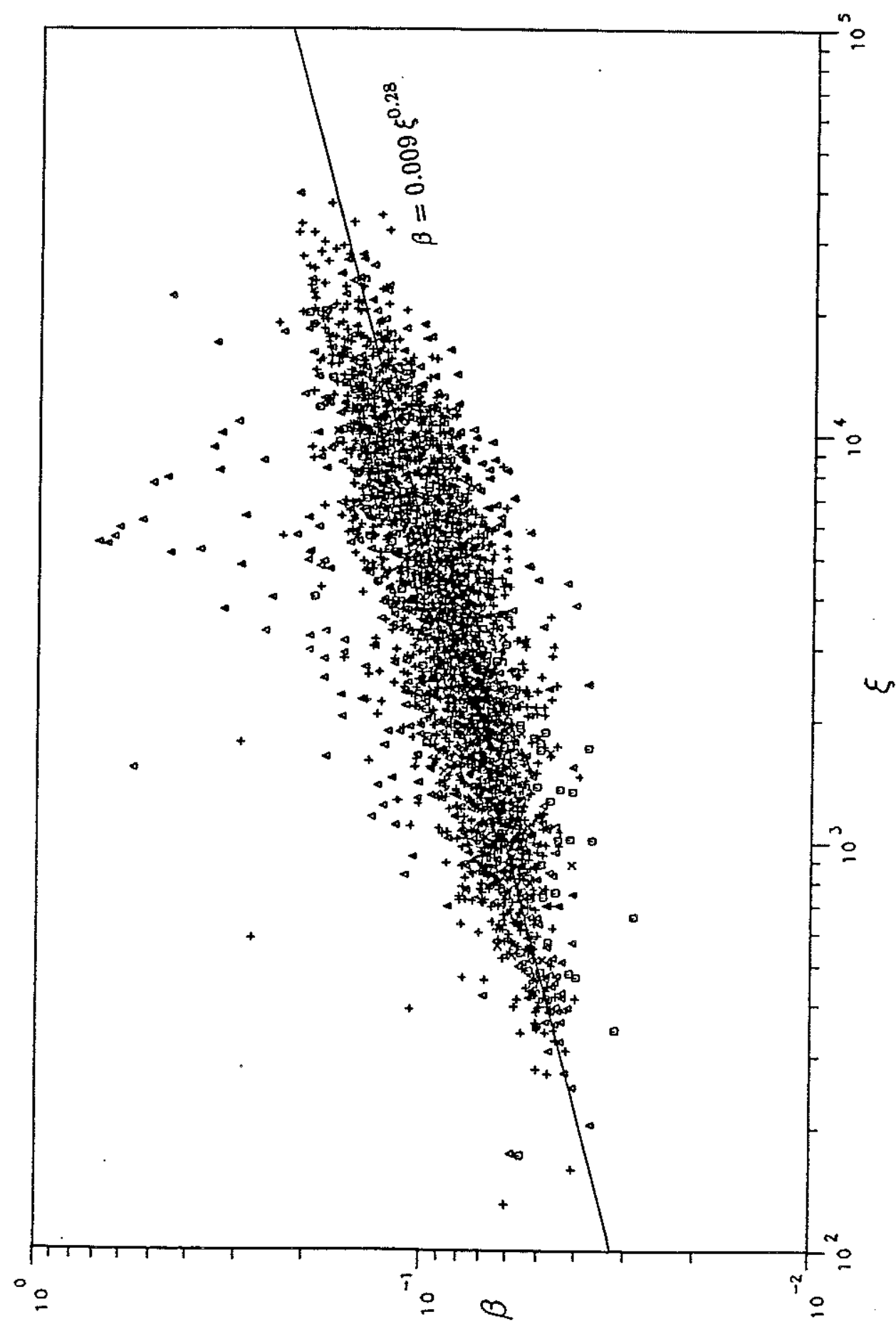

Figure 3-20: Equilibrium parameter $\beta$ versus dimensionless fetch $\xi$ with the regression relation $(3.54)$. 


\section{Chapter 4}

\section{Energy Balance}

In the previous chapter the shape of the wind-wave spectrum and the relations between spectral parameters, growth stage parameters and fetch were obtained on an empirical basis without considering the underlying physical processes that govern the evolution of the wave spectrum. For a growing wind- wave spectrum the energy input from wind peaks at a frequency slightly higher than the peak frequency of the spectrum. The energy transfer due to weakly nonlinear interactions between the various wave components moves energy from this peaked region to lower and higher frequencies, resulting in the migration of the spectral peak to lower frequencies. For an equilibrium state in deep water a balance exists between the wind input, nonlinear energy transfer and dissipation due to white-capping in the high-frequency range. In finite depth the low frequency wave components interact with the bottom and dissipate energy and as the waves propagate into shallower water more components feel the effects of bottom friction. When the energy dissipated by bottom friction is nearly equal to the low frequency energy transfer due to wave-wave interactions, the spectral peak no longer migrates towards the lower frequencies and may even move towards higher frequencies for higher dissipation rates. Bouws and Komen (1983) have shown in their analysis using storm data from the North Sea that the energy dissipated 
due to bottom friction is larger than the energy transfer due to the wave-wave interactions. Using data sets that indicate strong bottom dissipation the relative importance of wind input, nonlinear transfer, white-capping and bottom dissipation in finite depth is studied.

\subsection{The Energy Transport Equation}

The evolution of the spectrum can be described by the energy balance equation for the rate of change of the two-dimensional frequency spectrum $E(f, \theta)$

$$
\frac{\partial E}{\partial t}(f, \theta)+\nabla \cdot\left(c_{g} E\right)=S_{i n}+S_{n l}+S_{w b}+S_{b o t}
$$

where $\theta$ denotes the wave direction and $c_{g}$ is the group velocity of the waves with frequency $f$. The terms on the right indicate external energy sources and sinks where $S_{\text {in }}$ is the wind input, $S_{n l}$ is the nonlinear transfer due to wave-wave interactions, $S_{w b}$ is the energy lost due to white-capping and $S_{b o t}$ is the energy lost due interactions with bottom topography. The second term on the left acconts for the effects of a bottom slope on the spectrum and includes the effect of shoaling and refraction.

The functional form of the external source terms used in (4.1) are summarized based on previous studies. Snyder et al. (1981) proposed the following form for the wind input based on the parameterization of Miles' energy transfer mechanism

$$
\begin{aligned}
S_{\text {in }}(f, \theta) & =B \frac{\rho_{a}}{\rho_{w}} \omega\left[\frac{\mathbf{k} \cdot \mathbf{U}}{\omega}-1\right] E(f, \theta) & & \text { for } \frac{\mathbf{k} \cdot \mathbf{U}}{\omega}>1 \\
& =0 & & \text { for } \frac{\mathbf{k} \cdot \mathbf{U}}{\omega}<1
\end{aligned}
$$

where $\rho_{a}$ and $\rho_{w}$ refer to the densities of air and water, respectively. From direct measurements of the work done by wind-induced air pressure fluctuations over the sea surface the coefficient $B$ was determined to vary from 0.2 to 0.3 (Snyder et al., 1981). The above formulation shows that the wind speed in the direction of the waves should be greater than the phase speed of the waves for positive momentum transfer from wind to waves. Rewriting 
$\mathbf{k} \cdot \mathrm{U} / \omega$ as $U_{e} / c$, where $U_{e}$ is the component wind speed in the direction of waves, (4.2) becomes

$$
S_{\text {in }}(f, \theta)=\max \left\{0, B \frac{\rho_{a}}{\rho_{w}} \omega\left[\frac{U_{e}}{c}-1\right] E(f, \theta)\right\} .
$$

The wind input is sharply peaked and centered just around the spectral peak frequency for growing seas.

Hasselmann (1962) derived the energy flux in a gravity wave spectrum resulting from weakly nonlinear interactions between spectral components. The nonlinear transfer rate was expressed as a six-dimensional Boltzmann integral for a general spectrum. The energy transfer has been numerically calculated in many studies ( Hasselmann, 1963; Sell and Hasselmann, 1972; Webb, 1978; Hasselmann and Hasselmann, 1981; Weber, 1988). Since the numerical calculations require extensive computer time and memory, the transfer integral has been calculated for special wave spectra using narrow-peak approximations (Longuet-Higgins, 1976; Fox, 1976 ). Also, parametric representations of the interaction process (Barnett, 1966; Resio, 1981; Hasselmann and Hasselmann, 1981) have been proposed to evaluate the energy transfer. Hasselmann and Hasselmann (1981) calculated the exact transfer rate from the integral transfer equation for many wind-sea spectra and showed that the nonlinear transfer rate for any wind-sea spectrum is a distorted version of the transfer rate of a reference spectrum. The distortion is rectified by a transformation of the frequency axis and a scaling factor. The mean JONSWAP spectrum with peak enhancement factor $\gamma=3.3$ and equilibrium parameter $\alpha_{5}=0.01$ was used as the reference spectrum. For any general spectrum with $\gamma \neq 3.3$ the axis is transformed according to

$$
\nu^{*}=\Psi(\gamma)\left(\nu-\nu_{0}\right)+\nu_{0}^{*}
$$

where $\nu^{*}$ is the dimensionless frequency of the mean JONSWAP spectrum and $\nu$ is the dimensionless frequency of the wind-sea spectrum. For the mean JONSWAP spectrum the zero-transfer point of the energy transfer $\nu_{0}^{*}=1.0092$ and the zero-transfer point of the 
wind-wave spectrum $\nu_{\mathrm{o}}$ is a function of $\gamma$. The transfer rate $S_{n l}(\nu, \theta)$ is given by

$$
S_{n l}(\nu, \theta)=\Xi(\gamma) R\left(k_{m} h\right)\left(\frac{\alpha_{5}}{\alpha_{5}^{*}}\right)^{3}\left(\frac{f_{m}}{f_{m}^{*}}\right)^{-4} S_{n l}^{*}\left(\nu^{*}, \theta\right)
$$

where the starred variables indicate the values of the reference spectrum. $\Xi$ is a scaling factor and the factor $R\left(k_{m} h\right)$ is a factor introduced by Herterich and Hasselmann (1980) to account for finite depth effects in a narrow-band spectrum and is given by

$$
R(x)=1+\frac{5.5}{x}\left(1-\frac{5}{6} x\right) e^{-1.25 x}
$$

where $x=k_{m} h$ and $k_{m}=0.75 \bar{k}$ and $\bar{k}=\langle 1 / \sqrt{k}\rangle^{-2}$. The factors $\Psi, \Xi$ and $\nu_{\mathrm{o}}^{*}$ are functions of $\gamma$ and are given by Hasselmann and Hasselmann (1981).

Theoretical work on the spectral dissipation of waves due to white-capping was first presented by Hasselmann (1974). The dissipation was related to the wave spectrum linearly through a damping factor $\lambda_{d}$

$$
S_{w b}(f, \theta)=-\lambda_{d}(2 \pi f)^{2} E(f, \theta)
$$

and the factor $\lambda_{d}$ was estimated from the assumption that at high frequencies there should be a balance between wind input, nonlinear transfer and wave breaking. Bouws and Komen (1983) formulated a functional form similar to ( 4.7 ) but calculated $\lambda_{d}$ from the requirement that

$$
\sum S_{n e t}^{2}\left(f_{i}\right)=\text { minimum } \quad \text { for } 0.8 f_{m}<f_{i}<2 f_{m}
$$

such that

$$
\int_{0.8 f_{m}}^{2 f_{m}} S_{n e t} d f=0
$$

where $S_{n e t}$ is the sum of the source terms $S_{i n}, S_{n l}, S_{b f}$ and $S_{w b}$. Komen et al. (1984) generalized the form of wave dissipation as

$$
S_{w b}(\mathbf{k})=-c \bar{\omega}\left(\frac{\omega}{\bar{\omega}}\right)^{n}\left(\frac{\hat{\alpha}}{\hat{\alpha} P_{M}}\right)^{m} F(\mathbf{k})
$$


where $\hat{\alpha}=\tilde{E} \bar{\omega}^{4} / g^{2}$ and $\bar{\omega}=E^{-1} \int F(\mathbf{k}) \omega d \mathbf{k}$ and $\hat{\alpha}_{P M}=4.57 \times 10^{-3}$. It was determined that for $n=m=2$ and $c=3.33 \times 10^{-5}$ an equilibrium solution for the energy balance exists. The model (4.9) was transferred to frequency space by Janssen and Komen (1985) given by

$$
S_{w b}(f, \theta)=-4.3 \bar{\omega} \frac{k}{\bar{k}}\left[\tilde{E} \bar{k}^{2}\right]^{2} E(f, \theta)
$$

In this analysis the formulation (4.10) is used to calculate the energy dissipated due to white-capping.

In finite depth energy can be dissipated due to percolation, mean sea-level variations, wind-driven and tidal currents, depth-induced breaking and bottom friction. In this analysis, only the energy dissipated due to friction in the wave induced bottom turbulent boundary- layer is considered. Hasselmann and Collins (1968) derived a formulation to express spectral dissipation in terms of the wind-sea spectrum, bottom orbital speed and bottom roughness. Expressing the shear stress at the bottom with a quadratic friction law the energy dissipated was given by

$$
S_{b o t}(f, \theta)=-\frac{f_{w}}{2 g}\left(\frac{\omega}{\sinh k h}\right)^{2}<u_{b}>E(f, \theta)
$$

where $f_{w}$ is the friction factor characterizing bottom roughness and $\left\langle u_{b}\right\rangle$ is an average of bottom orbital speed $u_{b}$ given by

$$
<u_{b}>=\left\{2 \iint\left(\frac{\omega}{\sinh k h}\right)^{2} E(f, \theta) d f d \theta\right\}^{0.5} .
$$

Values for friction factors $f_{w}$ were obtained by introducing the concept of eddy viscosity to solve the dynamical equations of motion in the turbulent boundary-layer by Kajiura (1964) and Grant (1977). Grant and Madsen (1982), from analyses of laboratory data, identified two distinct contributions to the roughness in a unsteady oscillatory flow: the form drag due to the bedforms and the influence of the sediment transport near the bed. The relative 
bottom roughness $k_{b} / A_{b}$ is then given by

$$
\frac{k_{b}}{A_{b}}=15(s-1)\left\{\left(\frac{\psi^{\prime}}{\psi_{c}}\right)^{0.5}-0.7\right\}^{2} \frac{d}{A_{b}}+28 \frac{\eta}{A_{b}} \frac{\eta}{\lambda}
$$

where $\psi^{\prime} / \psi_{c}$ is ratio of the maximum values of Shields parameter $\psi^{\prime}$ to the critical value for intiation of motion $\psi_{c}$ and

$$
\psi^{\prime}=\tau_{b m}^{\prime} / \rho(s-1) g d
$$

$s=\rho_{s} / \rho$ is the relative density of the sediment particles, $d$ is the sediment diameter and the maximum shear stress due to skin friction $\tau_{b m}^{\prime}$ is obtained from

$$
\tau_{b m}^{\prime}=\frac{1}{2} \rho f_{w}^{\prime} u_{b}^{2}
$$

where the skin friction factor $f_{w}^{\prime}$ is obtained from the sand grain roughness. $\eta$ and $\lambda$ are the typical dimensions of the wave generated bedforms and are empirically expressed in terms of $\psi^{\prime} / \psi_{c}$. For a given wave condition the effective roughness can be calculated from (4.13) and the corresponding friction factor can be obtained from Grant (1977).

\subsection{Analysis and Discussion}

When the wind blows from around $290^{\circ}$ from true north the five towers $C 3, U 2, C 2, U 1$ and $C 1$ along the transect shown in Fig. 2-1 provide an ideal test case to study the effective balance of the various source terms in (4.1) for growing wind-seas in finite depth. Along the line of wave towers the water depth increases from the shallow regions near the northwest shore to $7 \mathrm{~m}$ at tower $U 2$ and decreases to $3.7 \mathrm{~m}$ at tower $C 1$. The bottom contours in the vicinity of the towers can be assumed to be normal to the line of wave towers and as a first approximation refraction effects are neglected for wave propagation along the line of the five wave towers. For a steady wind along the line of towers the spectral energy will increase and the spectral peak frequency will migrate towards the lower frequencies as energy is transferred to lower frequencies due to wave-wave interactions. As the waves 
move into shallower water the dissipation due to bottom friction increases and balances the nonlinear energy transfer. Then the wave energy is attenuated and the spectral peak stops migrating to lower frequencies and may even move towards higher frequencies for strong friction effects. The variation of energy and spectral peak frequency along the wave towers is presented in Table 4.1 for two data sets (hereafter referred to as Case 1 and Case 2) that had wind directions nearly coinciding with the line of wave towers. In Case 1 the wave height decreases from 0.84 to $0.67 \mathrm{~m}$ and the peak frequency increases from 0.28 to $0.32 \mathrm{~Hz}$ as the waves move from tower $U 2$ to tower $C 1$ indicating strong bottom friction dissipation. In Case 2 the peak frequency is still moving to the lower values $(0.26$ to $0.25 \mathrm{~Hz})$ and the wave height has decreased from $0.82 \mathrm{~m}$ at $U 2$ to $0.80 \mathrm{~m}$ at tower $C 1$. Case 1 measurements were made on 14th of November at 20:00 hrs and the wind speed was $12.3 \mathrm{~m} / \mathrm{s}$ and the wind direction $288^{\circ}$ from the true north. Case 2 measurements were made on 24 th of November at 12:00 hrs and the wind speed was $10.6 \mathrm{~m} / \mathrm{s}$ and wind direction $291^{\circ}$ from the true north. In Table 4.1 the relevant spectral parmeters for the wave towers are tabulated for the two cases.

The spectral measurements at tower $C 1$ are used to calculate the source terms $S_{i n}, S_{n l}$, $S_{w b}$ and $S_{b o t}$ as defined in Section 4.1. The spectra used for the calculations of the source terms for the two cases are presented in Fig. 4-1a and Fig. 4-2a. Using $B=0.3$ in (4.3) the wind input is calculated and is plotted in Fig. 4-1b and Fig. 4-2b for the two cases considered here. The energy is assumed to be in the same direction as the wind. The wind input is peaked near the spectral peak frequencies and falls very rapidly below the peak frequency. The growth of the forward face of the spectrum is due to energy transfer from higher to lower frequencies due to the wave-wave interactions. The nonlinear transfer $S_{n l}$ is obtained from the relation (4.5). The values of the peak enhancement factor for the spectra at tower $C 1$ obtained are $\gamma=3.2$ for Case 1 and $\gamma=1.2$ for Case 2. Using the $S_{n l}^{*}$ values calculated by Hasselmann and Hasselmann (1981) for the reference spectrum the 


\begin{tabular}{|c|c|c|c|c|c|}
\hline Tower & $\mathrm{C} 3$ & $\mathrm{U} 2$ & $\mathrm{C} 2$ & $\mathrm{U} 1$ & $\mathrm{C} 1$ \\
\hline Depth $(m)$ & 6.7 & 7.0 & 6.4 & 5.5 & 3.7 \\
\hline Fetch $(k m)$ & 19.0 & 27.6 & 31.8 & 35.9 & 39.6 \\
\hline \hline Case 1 & & & & & \\
\hline$H_{s}(m)$ & 0.72 & 0.84 & 0.79 & 0.77 & 0.67 \\
\hline$f_{m} H z$ & 0.29 & 0.28 & 0.28 & 0.29 & 0.32 \\
\hline$k_{m}\left(m^{-1}\right)$ & 0.34 & 0.32 & 0.33 & 0.35 & 0.44 \\
\hline$c_{g}(m / s)$ & 2.91 & 2.99 & 3.02 & 3.02 & 2.84 \\
\hline \hline Case 2 & & & & & \\
\hline$H_{s}(m)$ & 0.79 & 0.82 & & 0.82 & 0.80 \\
\hline$f_{m} H z$ & 0.28 & 0.26 & & 0.25 & 0.25 \\
\hline$k_{m}\left(m^{-1}\right)$ & 0.33 & 0.28 & & 0.27 & 0.30 \\
\hline$c_{g}(m / s)$ & 3.00 & 3.36 & & 3.71 & 3.78 \\
\hline
\end{tabular}

Table 4.1: Spectral parameters for Case 1 and Case 2 at the towers 
energy transfer calculated is plotted in Fig. 4-1c and Fig. 4-2c. For Case 2 the transfer across the peak is very small and this is due to the small peak enhancement factor $\gamma$ for the measured spectrum compared to the mean JONSWAP spectrum. Energy loss due to white-capping based on (4.10) is illustrated in Figs. 4-1d and 4-2d. The lake bottom near the wave towers $U 1$ and $C 1$ consists of medium and fine sand $\left(\phi<3 ; \phi=-\log _{2} d(m m)\right)$. A characterestic diameter of $0.2 \mathrm{~mm}$ is assumed to calculate the friction factor $f_{w}$ from (4.13). Using the wave conditions at tower $C 1$ the friction factors $f_{w}$ obtained from (4.13) for the two cases are 0.22 and 0.065 , respectively. The friction factors obtained here are one order of magnitude higher than the skin friction factor $f_{w}^{\prime}=0.01$ and include the effects of the drag due to ripples and sediment movement on the lake bed. The source term $S_{b o t}$ is calculated from (4.11) and plotted in Fig. 4-1e for Case 1 and in Fig. 4-2e for Case 2. The derivatives of $E(f, \theta), \partial E / \partial t$ and $\partial\left(c_{g} E\right) / \partial x$, where $x$ is the distance along the line of wave towers, are calculated using finite difference expressions

$$
\left.\frac{E_{2}-E_{1}}{\Delta t}\right|_{U 2}
$$

and

$$
\frac{\left.c_{g} E_{1}\right|_{C 1}-\left.c_{g} E_{1}\right|_{U 2}}{\Delta x}
$$

where the subscripts 1 and 2 refer to time 20:00 and 21:00 for Case 1 and 12:00 and 13:00 for Case 2. The distance between the towers $U 2$ and $C 1$ is about $12 \mathrm{~km}$ and for a representative group velocity of $3 \mathrm{~m} / \mathrm{s}$ the travel time between the two towers is about 70 minutes. Based on this travel time $\Delta t$ in (4.14) is taken as one hour. The time derivatives of the spectra estimated for Case 1 and Case 2 are shown in Figs. 4-1f and 4-2f. For a duration-limited growing wind-sea the saturation limit is attained when the time derivative is zero and the other terms in (4.1) are in a balance. For Case 1 there is a small change in energy content with time near the spectral peak region and an even smaller change for Case 2. The space derivative term which is due to the effect of the depth variation from tower $U 2$ to $C 1$ on 

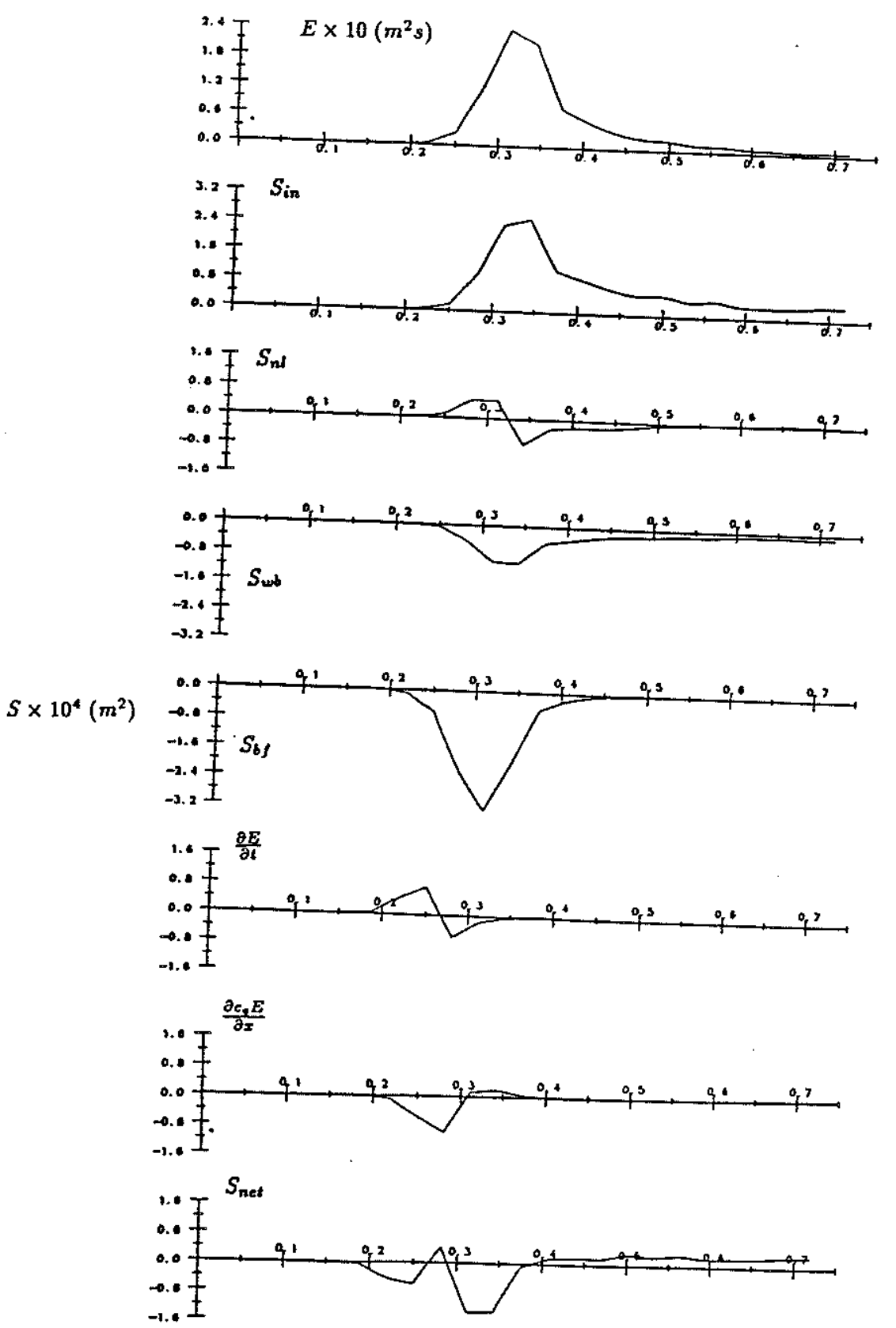

$$
f(H z)
$$

Figure 4-1: The spectrum and the various source terms contributing to the energy balance in the wind-sea at tower $C 1$ for Case 1. a) spectrum; b) wind input; c) nonlinear energy transfer; d) dissipation due to white-capping; e) bottom dissipation; $f$ ) time rate of change of energy; g) rate of change of energy flux in the direction of propagation; $h$ ) sum of the source and derivative terms. 


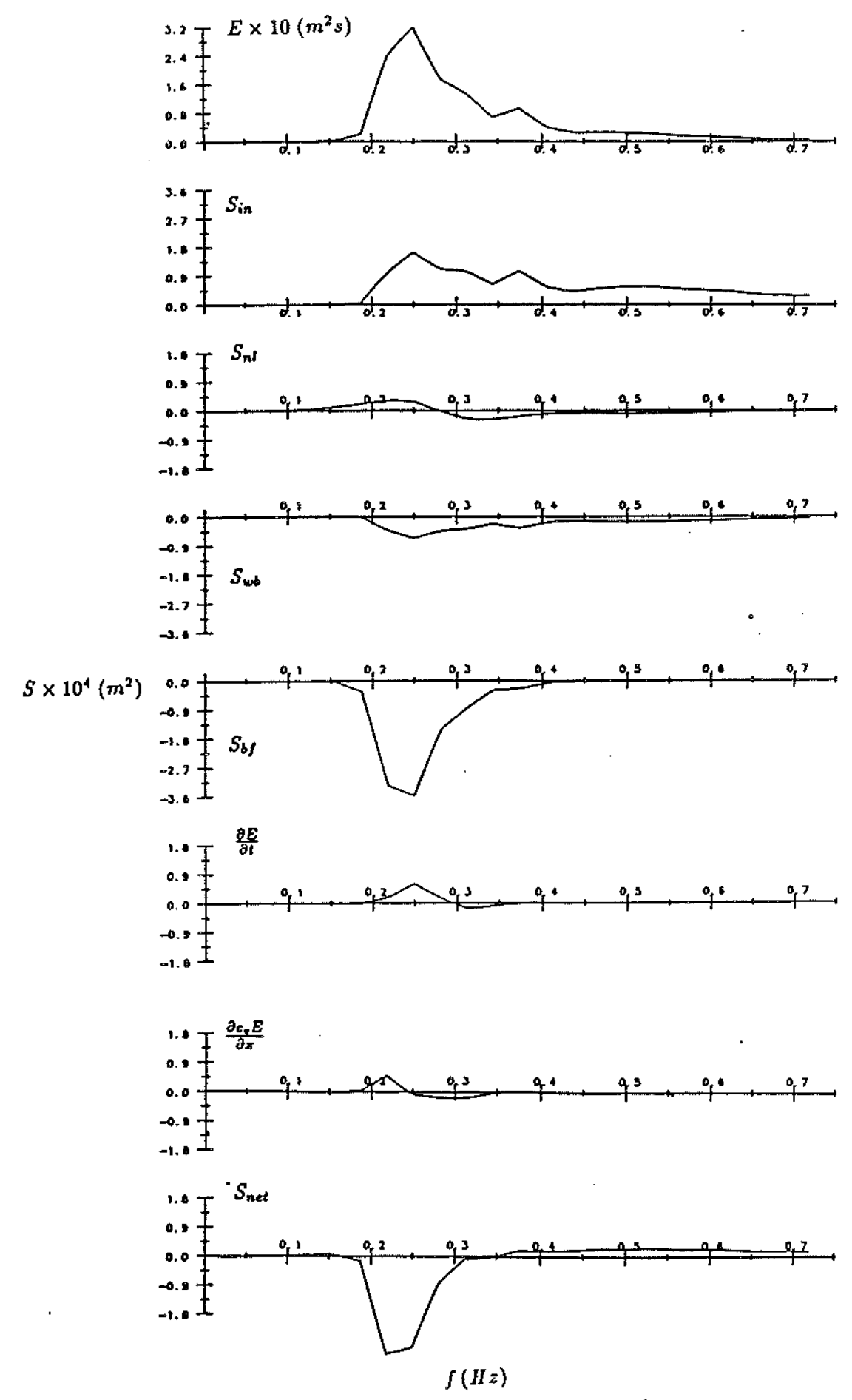

Figure 4-2: Same as Figure 4-1, but for Case 2 
the energy flux is shown for both the cases in Figs. 4-1f and 4-2f. The plots indicate that for Case 1 there is a decrease of energy flux for frequencies below the spectral peak and a small increase for frequencies higher than the spectral peak and vice-versa for Case 2. The relative importance of the source and derivative terms is evident in Figs. 4-1 and 4-2 for the two cases. The dominating terms in the energy balance are $S_{i n}$ and $S_{b f}$ with appreciable contibutions from the other terms for Case 1. The sum of all the source terms and derivatives, $S_{n e t}$

$$
S_{n e t}=S_{i n}+S_{n l}+S_{w b}+S_{b f}-\frac{\partial E}{\partial t}-\frac{\partial c_{g} E}{\partial x}
$$

is given in the Figures $4-1 \mathrm{~h}$ and $4-2 \mathrm{~h}$. It is seen that in both cases the $S_{n e t}$ terms are not close to zero, especially near the spectral peak frequencies and implies that there is no balance between various source terms. In both cases it seems that the bottom friction is overestimated which leads to large negative values of $S_{\text {net }}$ near the spectral peak. The net rate of change in energy due to the various source terms in the range $0.8 f_{m}$ to $2 f_{m}$ for the two cases are given below (units $m^{2} s^{-1}$ ) :

$$
\begin{array}{ccc} 
& \text { Case 1 } & \text { Case 2 } \\
\int S_{i n} d f & 3.47 \times 10^{-5} & 2.50 \times 10^{-5} \\
\int S_{n l} d f & -0.17 \times 10^{-5} & -0.04 \times 10^{-5} \\
\int S_{w b} d f & -1.35 \times 10^{-5} & -0.94 \times 10^{-5} \\
\int S_{b f} d f & -2.77 \times 10^{-5} & -3.19 \times 10^{-5} \\
\int \frac{\partial E}{\partial t} d f & 0.12 \times 10^{-5} & 0.21 \times 10^{-5} \\
\int \frac{\partial c_{g} E}{\partial x} d f & -0.38 \times 10^{-5} & 0.02 \times 10^{-5} \\
\int S_{n e t} d f & -0.56 \times 10^{-5} & -1.91 \times 10^{-5}
\end{array}
$$

The net rate of change in energy for the various source terms indicate the domination of the wind input and the dissipation terms. The net nonlinear transfer to higher and lower frequencies from the range $0.8 f_{m}<f<2 f_{m}$ is about one order of magnitude smaller than 
the wind input. It is seen that the space derivative of the energy flux is positive for Case 2 even though the energy is decreasing over the length. The small decrease in energy (from a wave height of $0.82 \mathrm{~m}$ at $U 2$ to $0.80 \mathrm{~m}$ at $C 1$ ) is overcome by the increase of group velocity $c_{g}$ to give a increase in energy flux.

To obtain a balance of the terms in (4.1) a greater wind input or a lower dissipation is needed. Since the value of the coefficient $B$ used to calculate the wind input is the largest in range of values determined by Snyder et al. (1981) a balance between the various terms is obtained with new estimates of the bottom dissipation. The friction factor $f_{w}$ is considered as a free parameter and calculated such that

$$
\int_{0.8 f_{m}}^{2 f_{m}} S_{n e t} d f=0
$$

New values of the friction factor obtained are

$$
\begin{aligned}
& f_{w}=0.18 \quad \text { for Case } 1 \\
& f_{w}=0.02 \quad \text { for Case } 2
\end{aligned}
$$

The new values of $f_{w}$ are about 80 and 30 of the values found from the Grant and Madsen (1982) approach. The bottom roughness in the Grant and Madsen approach is a function of the ripple dimensions and the variability in the friction factors obtained here may be because the ripples did not develop to their maximum dimensions for the duration in which the given wave conditions are present.

The various source terms using the new friction factors are given in Figs. 4-3 and 4-4 for the two cases studied. The wind input is the dominant term, but is balanced by the dissipation due to white-capping and bottom friction. The nonlinear term is very small and so is the rate of change of energy with time. In Case 2 an increase in energy flux as waves move from $U 2$ to $C_{1}$ is observed indicating that the net rate of change of the source terms $S_{i n}, S_{n l}, S_{w b}$ and $S_{b f}$ is positive. 

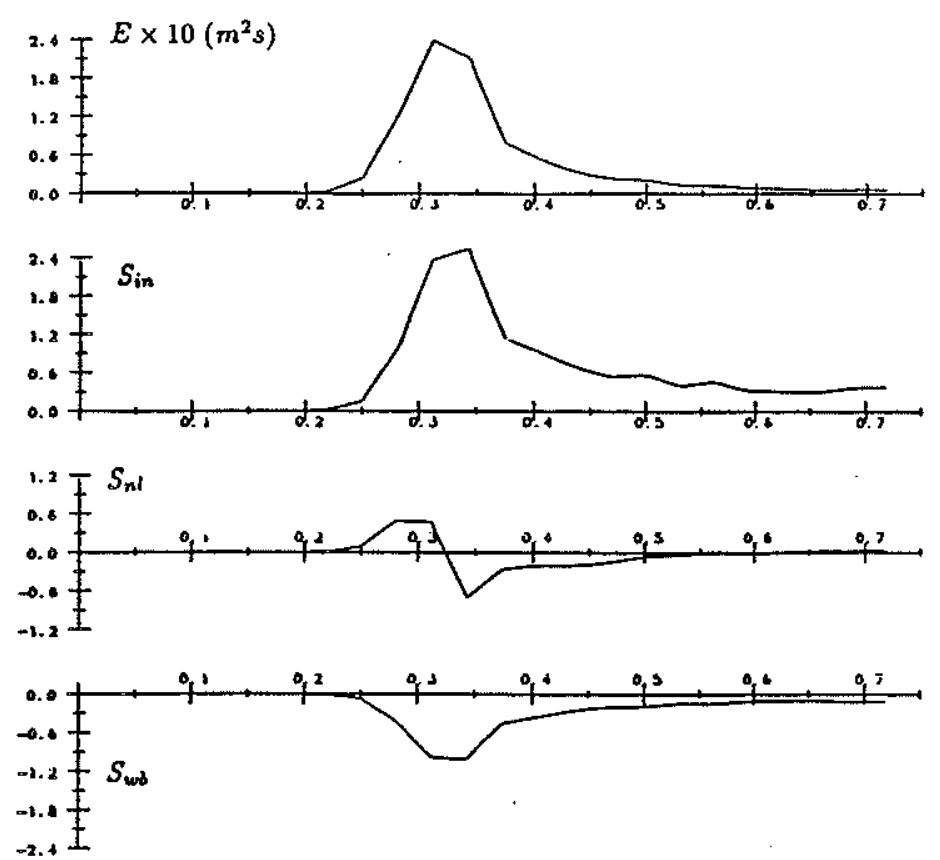

$S \times 10^{4}\left(m^{2}\right)$
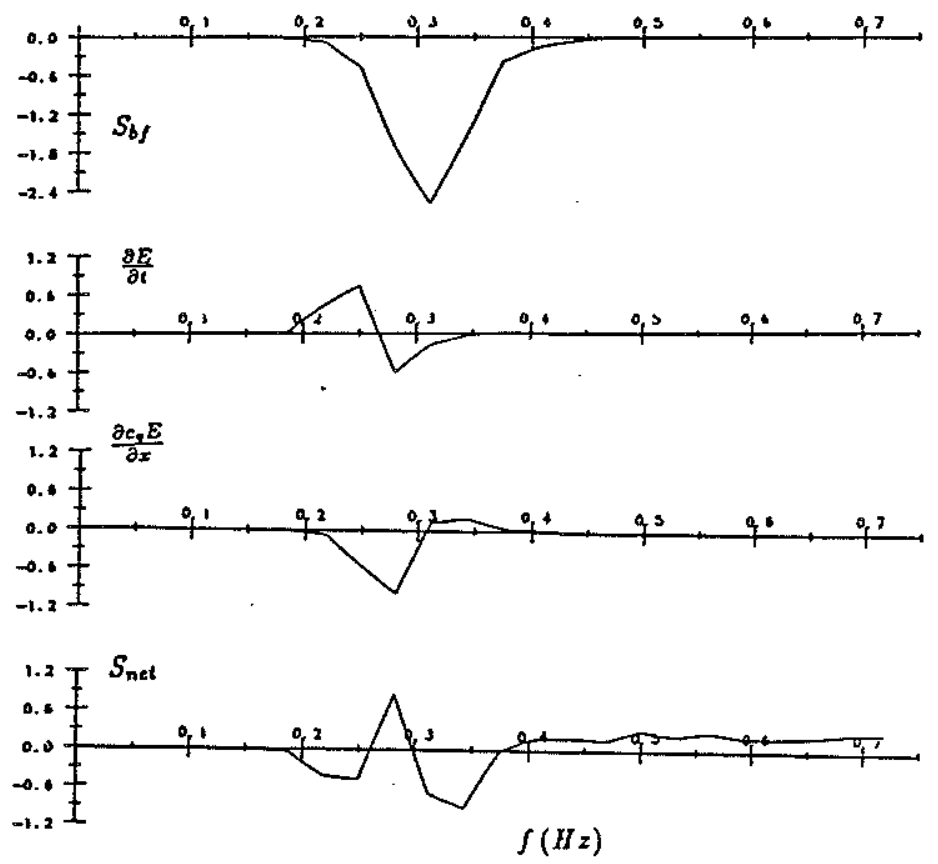

Figure 4-3: The spectrum and the various source terms contributing to the energy balance in the wind-sea at tower $C 1$ for Case 1 with new friction factor. a) spectrum; b) wind input; c) nonlinear energy transfer; d) dissipation due to white-capping; e) bottom dissipation; f) time rate of change of energy; g) rate of change of energy flux in the direction of propagation; $h$ ) sum of the source and derivative terms. 


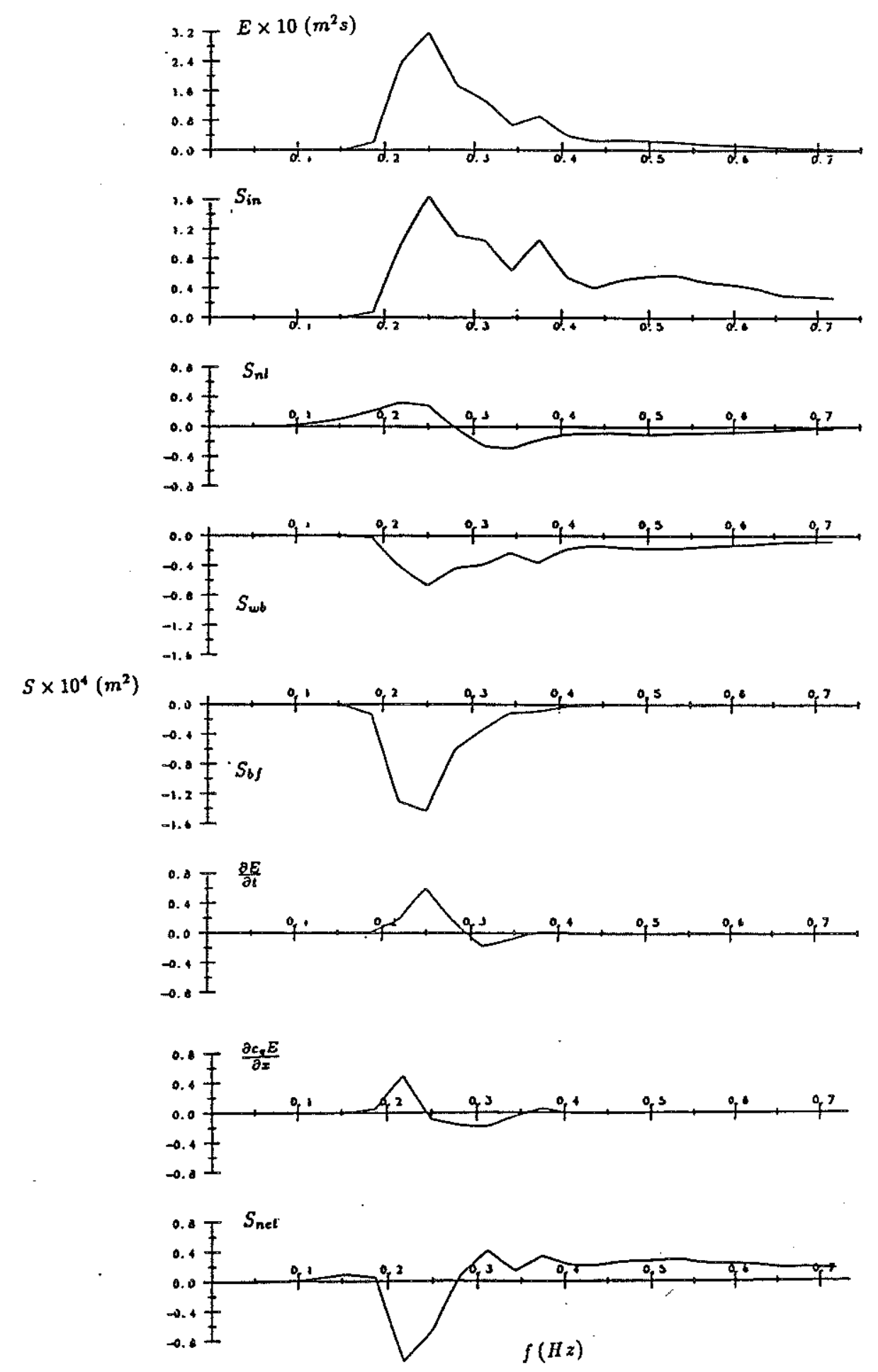

Figure 4-4: Same as Figure 4-3, but for Case 2 


\section{Chapter 5}

\section{Conclusions}

The objective of this study was to analyze the effects of finite depth on various aspects of the growth of a wave spectrum. The data is from a field program conducted by the Canadian Centre of Inland Water and the Great Lakes Environmental Research Laboratory in Lake St. Clair, a shallow fetch-restricted lake on the Canada - U.S border.

Observations under fetch-restricted conditions have shown that the wind direction and the wave propagation direction do not coincide. The wave propagation direction depends on an effective fetch which, in this case, is determined by the geometry of the lake. The relative angle between the wind and wave propagation directions is calculated based on an effective fetch formulation proposed by Donelan (1980). In regions where the fetch varies appreciably relative angles up to $60^{\circ}$ are observed. Due to an unresolved error in the analysis of the measured wave direction at the CCIW towers, the predicted values could not be compared to field measurements.

The shape of the rear face of a wind spectrum is expressed in three different functional forms based on Phillips (1958), Toba (1972) and Donelan et al. (1985). The equilibrium parameters $\alpha_{5}, \beta$ and $\alpha_{4}$ for the three formulations, respectively, are calculated from mea-

sured spectra. Based on an assumption of similarity of the spectral shape in wave number 
space, relationships between the nondimensional energy $\epsilon$ and $\alpha_{4} \kappa_{m}^{-2}, \beta \kappa_{m}^{-1.5}$ and $\alpha_{5} \kappa_{m}^{-2}$ are derived for the three forms. Relationships between energy and the equilibrium parameters for the data set are obtained by a regression analysis and compared to the theoretical relations. The fit of the regression relations to the theoretical relations demonstrate that the spectral forms based on a $f^{-4}$ dependence on frequency (Toba, 1973; Donelan et al., 1985) are a better choice to represent the spectrum than a $f^{-5}$ dependence (Phillips, 1958). The data is classified into four ranges based on $\omega_{h}$, the dimensionless depth, to identify the effect of finite depth. For a $f^{-5}$ spectrum and Phillips' $\alpha_{5}$ formulation, Bouws et al. (1985) had observed nearly uniform relations between $\epsilon$ and $\alpha_{5} \kappa_{m}^{-2}$ for all depth ranges. The regression coefficients obtained in this study for the range $\omega_{h}<1.0$ differ considerably from the coefficients determined in the ranges for $\omega_{h} \geq 1$. The deviation of the regression relation in the latter range indicates a weak depth dependence for all the three formulations.

An interesting observation is that when the wind component in the direction of waves is used in the calculation of $\epsilon$ and $\kappa_{m}$ the regression relations in the range $\omega_{h} \leq 1.3$ move away from and for $\omega_{h}>1.3$ approach the theoretical relation. The wave directions for finite depth based on Donelan's (1980) effective fetch may be in slight error because the effective fetch formulation proposed by Donelan (1980) was derived based on the peak frequency growth in deep water. For example, depth or current refraction could significantly affect the resulting effective fetch.

The fit of the regression relations to the theoretical relations support the use of a single equation to describe the spectral form for a growing wind-sea in finite depth. The spectrum can be completely specified by the peak frequency, the energy and the equilibrium parameters. To estimate the parameters from observations, relations between the parameters and growth-stage variables are determined. The relations between nondimensional energy $\epsilon$ and $\nu$ and $U / c$ do not compare well with the results of Bouws et al. (1987) and they exhibit considerable variations with depth. The relation betweem $\epsilon$ and $\kappa_{m}$, the dimensionless wave 
number, compares well with the results of Bouws et al. (1987) and is nearly uniform over the depth range. Hence $\kappa_{m}$ will be a convenient parameter to describe the development of wind-sea spectra in finite depth. The variation of $\epsilon$ and $\nu$ with fetch are close to the observations of Hasselmann et al. (1973) and Donelan et al. (1985) for deep water. The value of $\alpha_{4}$ for a given fetch is smaller than the value observed by Donelan et al. (1985).

In this analysis efforts were made to include only data that represented cases of a pure growing wind-sea. All the data for which the measured wind speed and wave height were below $3 \mathrm{~m} / \mathrm{s}$ and $0.25 \mathrm{~m}$, respectively, were excluded. Most of the cases in which sudden shifts in wind direction had occurred and disturbances from other sources appear to dominate were also excluded and this resulted in reducing significantly the scatter in the figures.

The energy balance in two cases of growing wind-seas in finite depth is studied using various formulations for the source terms. As the estimates of the source terms are only approximate there are several sources of error which contribute to the energy balance. For example, the constant $B$ for the wind input term (4.3) has been found to vary up to $50 \%$. The empirical coefficients used in the parameterization of the dissipation due to white-capping have not been tested under wide range of conditions. The uncertainty in the friction factor may be large since no measurements were available of the sediment grain size distribution and the ripple geometry.

The bottom dissipation term, based on friction factors from Grant and Madsen (1982) was one of the dominanting source terms in the energy balance. Large values of $S_{\text {net }}$ in the spectral peak region indicated a lack of energy balance. Treating the friction factor as a free parameter and requiring a balance in the frequency range $0.8 f_{m}<f<2 f_{m}$ new friction factors were obtained. The factors are about $80 \%$ and $30 \%$ of the calculated values using the Grant and Madsen (1982) approach for the two cases. The variability in the friction factors may be the result of the short duration in which the ripples have to reach 
an equilibrium state corresponding to the wave conditions.

The other dominant term is the wind input, which is balanced by the dissipation due to white-capping and bottom friction. The rate of change of energy in time is very small indicating a nearly saturated growth situation. The rate of change of energy flux over the distance between the towers is small but not negligible. The total nonlinear transfer in the range $0.8 f_{m}<f<2 f_{m}$ is very small and so is the transfer across the peak. Thus for a growing wind-sea in finite depth the shape of the spectrum is largely controlled by wind input and dissipation due to bottom friction and white-capping. 


\section{References}

Barnett, T. P., 1968: On the generation, dissipation and prediction of ocean wind waves. J. Geophys. Res., 73(2), 513-529.

Battjes, J. A., T. J. Zitman and L. H. Holthuijsen, 1987: A reanalysis of the spectra observed in JONSWAP. J. Phys. Oceanogr., 17, 1288-1295.

Bouws, E., H. Günther, W. Rosenthal and C. L. Vincent, 1985: Similarity of the wind wave spectrum in finite depth water. Part 1: Spectral form. J. Geophys. Res., 90(C1), 975-986.

Bouws, E., H. Günther, W. Rosenthal and C. L. Vincent, 1987: Similarity of the wind wave spectrum in finite depth water. Part 2: Statistical relations between shape and growth stage parameters. Dt. Hydrogr. Z, 40, 1-24.

Bouws, E. and G. J. Komen, 1983: On the balance between growth and dissipation in an extreme depth-limited wind-sea in the southern North Sea. J. Phys. Oceanogr., 13, 1653-1658.

Donelan, M. A., 1980: Similarity theory applied to the forecasting of wave heights, periods and directions. Proc. of the Canadian Coastal Conf., National Research Council, Ottawa, Ontario, 47-61.

Donelan, M. A., J. Hamilton and W. H. Hui, 1985: Directional spectra of wind generated waves. Phil. Trans. Roy. Soc. London, A135, 509-562.

Forristal, G. Z., 1981: Measurements of a saturated range in ocean wave spectra. $J$. Geophys. Res., 86(C9), 8075-8084.

Fox, M. J. H., 1976: On the nonlinear transfer of energy in the peak of a gravity-wave spectrum - II. Proc. Roy. Soc. London, Ser. A 348, 467-483. 
Goda, Y., 1975: Irregular wave deformation in the surf zone. Coastal Engr. in Japan, $18,13-26$.

Grant, W. D., 1977: Bottom friction under waves in the presence of a weak current: its relationship to sediment transport. Sc. D. Thesis, Massachusetts Institute of Technology, 275pp.

Grant, W. D. and O. S. Madsen, 1982: Movable bed roughness in unsteady oscillatory flow. J. Geophys. Res., 87(C1), 469-481.

Hasselmann, K., 1962: On the nonlinear energy transfer in a gravity wave spectrum. Part 1: General theory. J. Fluid: Mech., 12, 481-500.

Hasselmann, K., 1963: On the nonlinear energy transfer in a gravity wave spectrum. Part 3: Evaluation of the energy flux and swell-sea interaction for a Neumann spectrum. J. Fluid. Mech., 15, 385-398.

Hasselmann, K., T. P. Barnett, E. Bouws, H. Carlsen, D. E. Cartwright, K. Enke, J. A. Ewing, H. Gienapp, D. E. Hasselmann, P. Kruseman, A. Meerburg, P. Müller, D. J. Olbers, K. Richter, W. Sell, and H. Walden, 1973: Measurements of wind wave growth and swell decay during the Joint North Sea Wave Project (JONSWAP). Dtsch. Hydrogr. Z., Suppl.A, No. 12.

Hasselmann, K. and J. I. Collins, 1968: Spectral dissipation of finite depth gravity waves due to turbulent bottom friction. J. Marine. Res., 26, 1-12.

Hasselmann, K., D. B. Ross, P. Müller and W. Sell, 1976: A parametric wave prediction model. J. Phys. Oceanogr., 6, 200-228.

Hasselmann, S. and K. Hasselmann, 1981: A symmetrical method of computing the nonlinear transfer in a gravity wave spectrum. Hamburger Geophys. Einzel., No. 52. 
Herterich, K. and K. Hasselmann, 1980: A similarity relation for the nonlinear energy transfer in a finite depth gravity wave spectrum. J. Fluid Mech., 97, 215-224.

Janssen, P. A. E. M. and G. Komen, 1985: A shallow water extension of the 3-GWAM model. Unpublished manuscript.

Kahma, K. K., 1981: A study of the growth of the wave spectrum with fetch. J. Phys. Oceanogr., 11, 1503-1515.

Kawai, S., K. Okada and Y. Toba, 1977: Field data support of three-seconds power law and $g u_{*} \sigma^{-4}$ spectral form for growing wind waves. J. Oceanogr. Soc. Japan, 33, 137-150.

Kitaigorodskii, S.A.,1983: On the theory of the equilibrium range in the spectrum of wind generated gravity waves. J. Phys. Oceanogr., 13, 816-827.

Kitaigorodskii, S.A., V. P. Krasitskii and M. M. Zaslavskii, 1975: On Phillips' theory of equilbrium range in the spectra of wind-generated gravity waves. J. Phys. Oceanogr., 5, 410-420.

Knowles, C. E., 1982: On the effects of finite depth on wind wave spectra: 1. A comparison with deep water equilibrium-range slope and other spectral parameters. J. Phys. Oceanogr., 12, 556-568.

Komen, G. J., S. Hasselmann and K. Hasselmann, 1984: On the existence of a fully developed wind-sea spectrum. J. Phys. Oceanogr., 14, 1271-1285.

Longuet-Higgins, M. S., 1976: On the nonlinear transfer of energy in the peak of a gravity-wave spectrum; A simplified model. Proc. Roy. Soc. London, Ser. A 347, 314-328. 
Müller, P., 1976: Parametrization of one-dimensional wind wave spectra and their dependence on the state of development. Hamburger Geophys. Einzel., No. 31.

Phillips, O. M., 1958: The equilibrium range in the spectra of wind generated waves. J. Fluid Mech., 4, 426-434.

Phillips, O.M., 1985: Spectral and statistical properties of the equilibrium range in wind generated gravity waves. J. Fluid Mech., 156, 505-531.

Pierson, W. J., and L. Moskowitz, 1964: A proposed spectral form for fully developed wind seas based on the similarity theory of S. A. Kitaigorodskii. J. Geophys. Res., 69(24), 5181-5190.

Resio, D. T., 1981: The estimation of wind-wave generation in a discrete spectral model. J. Phys. Oceanogr., 11, 510-525.

Schwab, D. J., J. R. Bennett, P. C. Liu and M. A. Donelan, 1984: Application of a simple numerical wave prediction model to Lake Erie. J. Geophys. Res., 89(C3), 3586-3592.

Sell, W. and K. Hasselmann, 1972: Computations of nonlinear energy transfer for JONSWAP and empirical wind wave spectra. Unpubl. MS thesis, Inst. Geophys., University of Hamburg.

Snyder, R. L., F. W. Dobson, J. A. Elliott and R. B. Long, 1981: Array measurements of atmospheric pressure fluctuations above surface gravity waves. J. Fluid. Mech., 102, 1-59.

SWAMP Group, 1985. OCEAN WAVE MODELLING, I. The Sea Wave Modelling Project (SWAMP): Principal results and conclusions., Plenum Press, New York, 256 pp. 
SWIM Group: E. Bouws, J. A. Ewing, J. Ephraums, P. Francis, H. Günther, P. A. E. M. Janssen, G. J. Komen, W. Rosenthal and W. J. P. de Voogt, 1985. Shallow water intercomparison of wave prediction models. Quart. J. Roy. Meteorol. Soc., 111, 1087-1112.

Toba, Y., 1972: Local balance in the air-sea boundary processes. I. J. Oceanogr. Soc. Japan, 28, 109-121.

Toba, Y., 1973: Local balance in the air-sea boundary processes. III. J. Oceanogr. Soc. Japan, 29, 209-220.

Venkatesh, S., M. Donelan, H. Graber, F. Liu, D. Schwab and M. Skafel, 1987: Finite depth waves - A preliminary analysis of data from a field study on lake St. Clair. Proc. of the Conf. on Ocean-Atmosphere interaction, Anaheim, CA., 154-156.

Webb, D. J., 1978: Nonlinear transfer between sea waves. Deep-Sea Res., 25, 279-298.

Weber, S. L., 1988: The energy balance of finite depth gravity waves. J. Geophys. Res., 93(C4), 3601-3607. 